\title{
Virtual Reality Simulator for Training in Myringotomy with Tube Placement
}

Caiwen Huang, The University of Western Ontario

Supervisor: Hanif. M. Ladak, The University of Western Ontario

A thesis submitted in partial fulfillment of the requirements for the Doctor of Philosophy degree in Electrical and Computer Engineering

(C) Caiwen Huang 2016

Follow this and additional works at: https://ir.lib.uwo.ca/etd

Part of the Other Computer Engineering Commons

\section{Recommended Citation}

Huang, Caiwen, "Virtual Reality Simulator for Training in Myringotomy with Tube Placement" (2016).

Electronic Thesis and Dissertation Repository. 3637.

https://ir.lib.uwo.ca/etd/3637

This Dissertation/Thesis is brought to you for free and open access by Scholarship@Western. It has been accepted for inclusion in Electronic Thesis and Dissertation Repository by an authorized administrator of Scholarship@Western. For more information, please contact wlswadmin@uwo.ca. 


\begin{abstract}
Myringotomy refers to a surgical incision in the eardrum, and it is often followed by ventilation tube placement to treat middle-ear infections. The procedure is difficult to learn; hence, the objectives of this work were to develop a virtual-reality training simulator, assess its face and content validity, and implement quantitative performance metrics and assess construct validity.
\end{abstract}

A commercial digital gaming engine (Unity3D) was used to implement the simulator with support for 3D visualization of digital ear models and support for major surgical tasks. A haptic arm co-located with the stereo scene was used to manipulate virtual surgical tools and to provide force feedback.

A questionnaire was developed with 14 face validity questions focusing on realism and 6 content validity questions focusing on training potential. Twelve participants from the Department of Otolaryngology were recruited for the study. Responses to 12 of the 14 face validity questions were positive. One concern was with contact modeling related to tube insertion into the eardrum, and the second was with movement of the blade and forceps. The former could be resolved by using a higher resolution digital model for the eardrum to improve contact localization. The latter could be resolved by using a higher fidelity haptic device. With regard to content validity, $64 \%$ of the responses were positive, $21 \%$ were neutral, and $15 \%$ were negative.

In the final phase of this work, automated performance metrics were programmed and a construct validity study was conducted with 11 participants: 4 senior Otolaryngology consultants and 7 junior Otolaryngology residents. Each participant performed 10 
procedures on the simulator and metrics were automatically collected. Senior Otolaryngologists took significantly less time to completion compared to junior residents. Junior residents had 2.8 times more errors as compared to experienced surgeons. The senior surgeons also had significantly longer incision lengths, more accurate incision angles, and lower magnification keeping both the umbo and annulus in view. All metrics were able to discriminate senior Otolaryngologists from junior residents with a significance of $\mathrm{p}<0.002$.

The simulator has sufficient realism, training potential and performance discrimination ability to warrant a more resource intensive skills transference study.

Keywords: myringotomy with tube placement, virtual reality, surgical simulation, face validity, content validity, performance metrics, construct validity 


\section{Co-Authorship}

This thesis is an integration of three articles, each constituting a chapter; the journal publications listed in the following are either accepted for publication or under revision.

Chapter 2: C. Huang, S. K. Agrawal, H. M. Ladak, "A virtual-reality simulator for training in myringotomy with tube placement," Journal of Medical and Biological Engineering, accepted April, 2015.

My contributions to this work included developing a geometric model of the ear; designing, implementing and testing the simulator and writing the manuscript. The work was motivated by S. Agrawal and H. Ladak who supervised all aspects of the work and edited the manuscript.

Chapter 3: C. Huang, H. Cheng, Y. Bureau, S. K. Agrawal, H. M. Ladak, "Face and content validity of a virtual-reality simulator for myringotomy with tube placement," Journal of Otolaryngology-Head and Neck Surgery, accepted October, 2015.

My contributions for this chapter included designing the questionnaire for assessing face and content validity in consultation with instructing surgeons, collecting data from participants, analyzing the data, and writing the manuscript. $\mathrm{H}$. Cheng provided suggestions for conducting the validity study and scheduled participants. Y. Bureau verified statistical calculations. All authors reviewed and edited the manuscript. The work was performed under the supervision of S. Agrawal and H. Ladak.

Chapter 4: C. Huang, H. Cheng, Y. Bureau, H. M. Ladak, S. K. Agrawal, "Automated quantitative metrics in a virtual-reality myringotomy and tube simulator: Development and construct validity, The Laryngoscope, Submitted in December, 2015.

My contributions to this chapter included designing metrics and implementing them in software, collecting data from participants, analyzing the data and writing the manuscript. S. Agrawal assisted with the identification of metrics and potential participants. H. Ladak 
guided the implementation of the metrics. H. Cheng helped design the study protocol and scheduled participants. Y. Bureau verified the statistical analysis. All co-authors reviewed and edited the manuscript. The work was performed under the supervision of S. Agrawal and H. Ladak. 


\section{Acknowledgments}

I would like to acknowledge my supervisor Dr. Hanif Ladak for his substantial guidance and direction for this project and co-supervisor Dr. Sumit Agrawal for his input in the design and validation of the simulator. I also thank Dr. Yves Bureau for his help in statistical analysis for the validity studies; Horace Cheng, a surgical resident, for his help in performing all validation studies; and Alireza Rohani for his help with the creation of the ear model.

Financial support for this project was provided by the Natural Sciences and Engineering Research Council of Canada (NSERC), Medtronic of Canada Ltd., the Ontario Research Fund (ORF), and Mitacs. 


\section{Table of Contents}

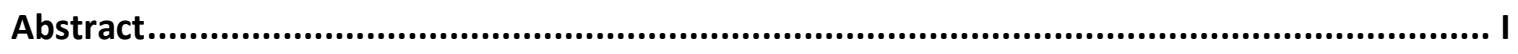

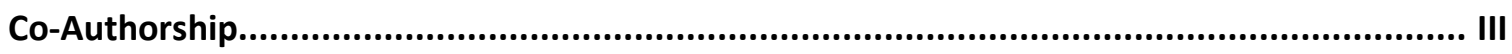

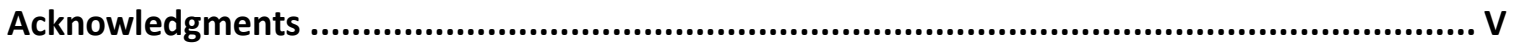

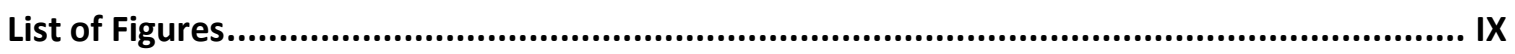

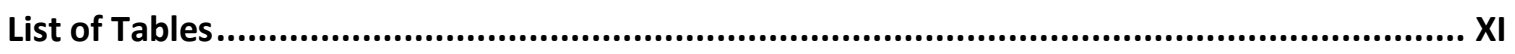

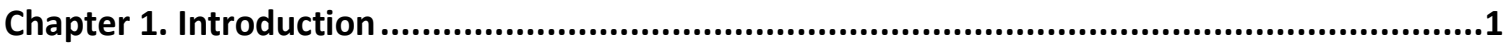

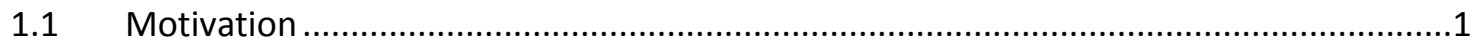

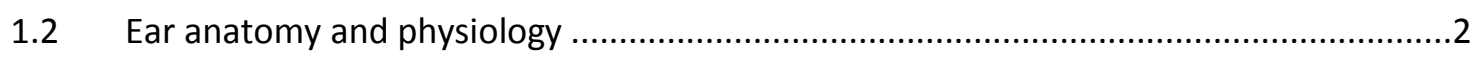

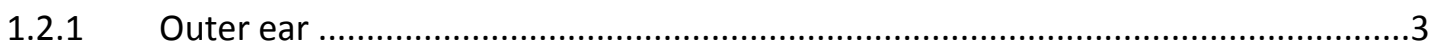

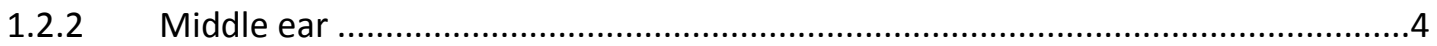

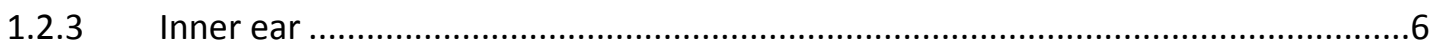

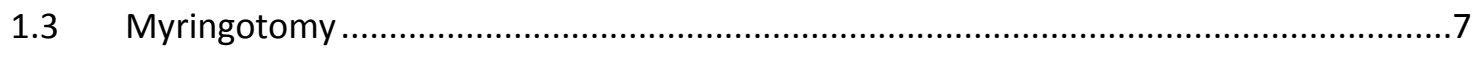

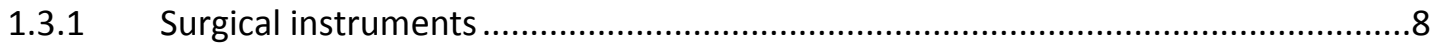

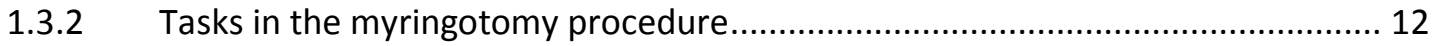

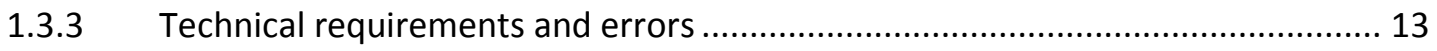

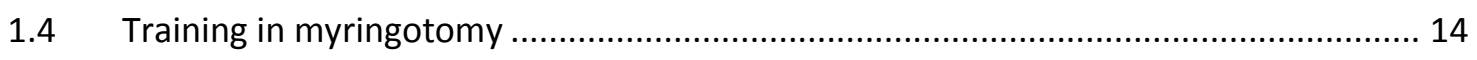

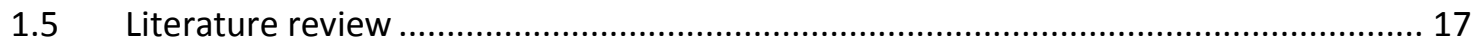

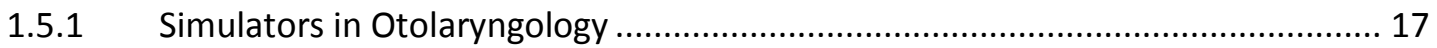

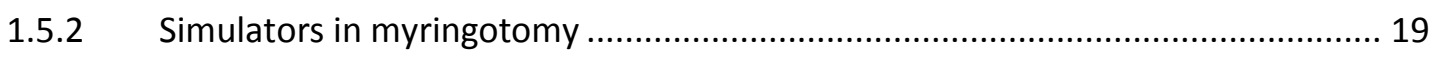

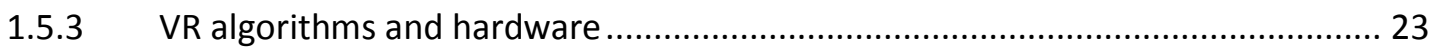

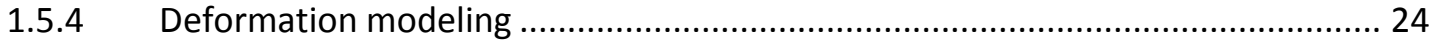

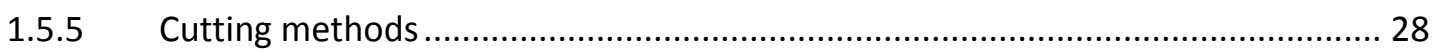

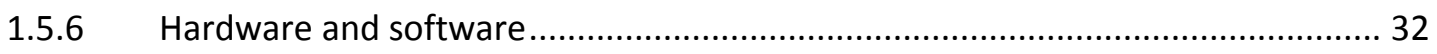

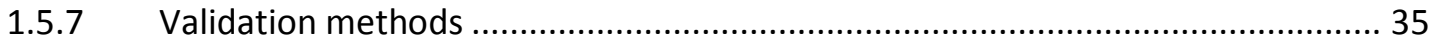

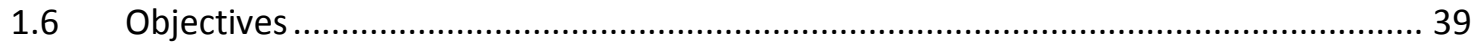

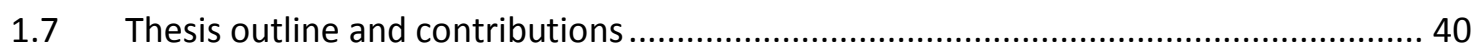

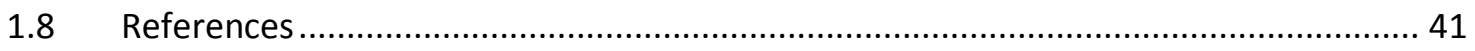

Chapter 2. Virtual-Reality Simulator for Training in Myringotomy with Tube Placement ........48

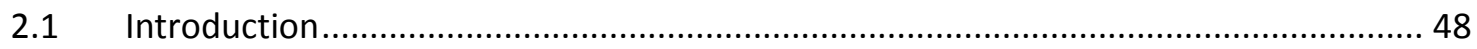

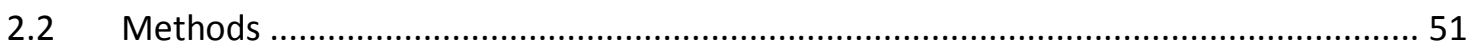




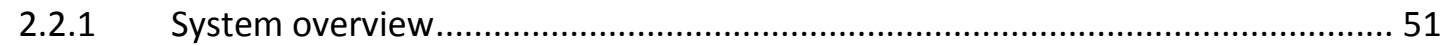

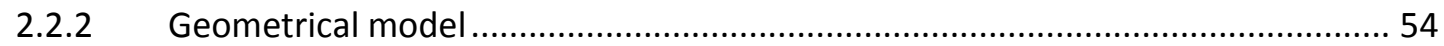

2.2.3 Deformation and collision models for the eardrum ......................................... 56

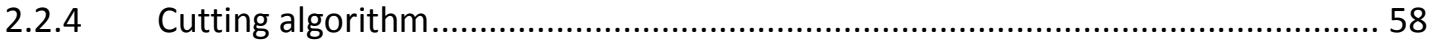

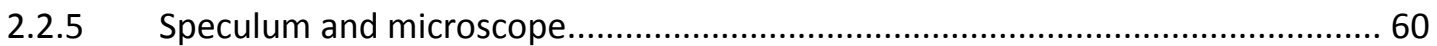

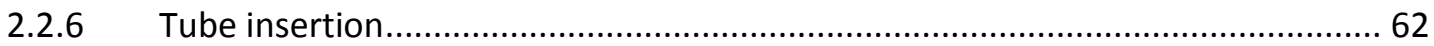

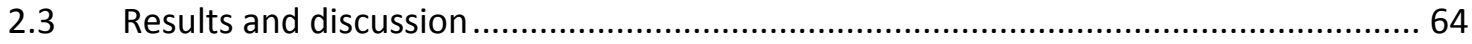

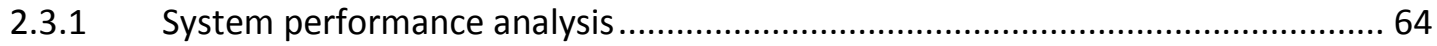

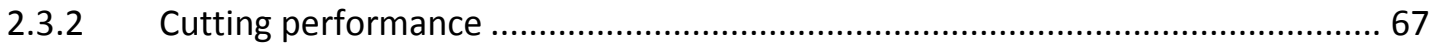

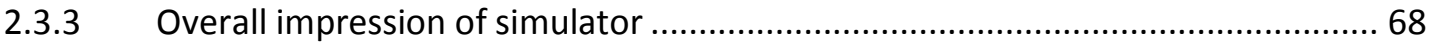

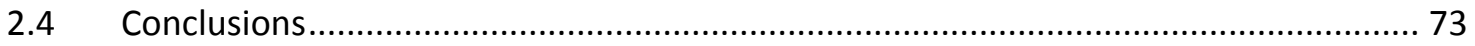

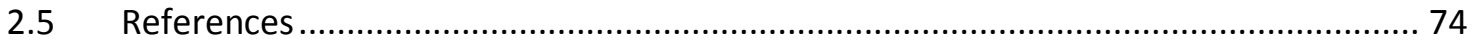

Chapter 3. Face and Content Validity of a Virtual-Reality Simulator for Myringotomy with

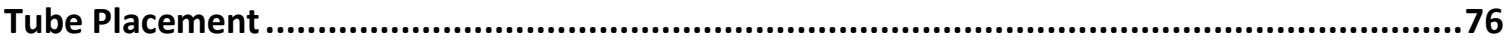

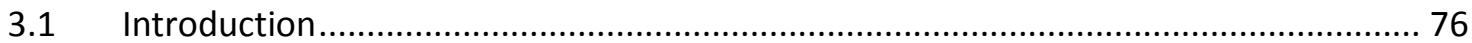

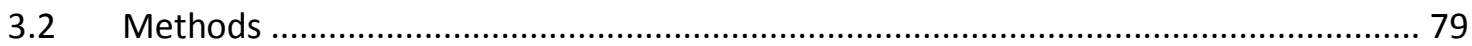

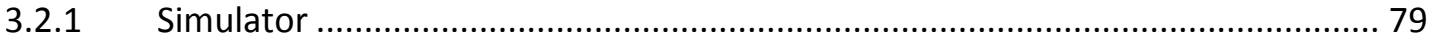

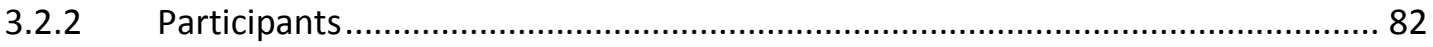

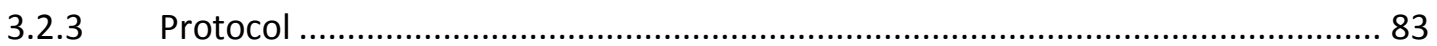

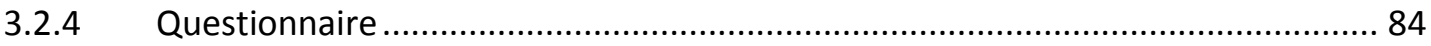

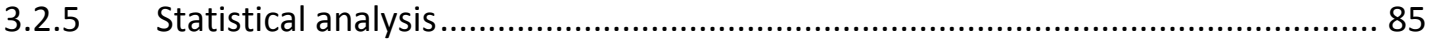

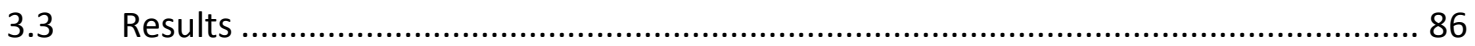

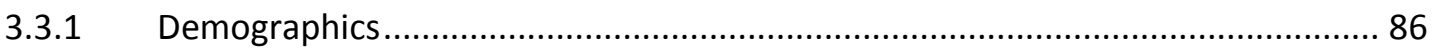

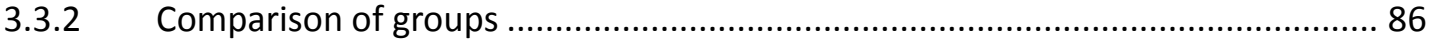

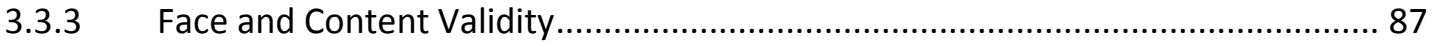

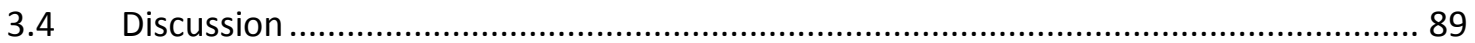

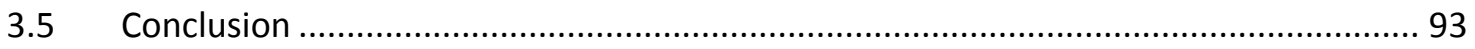

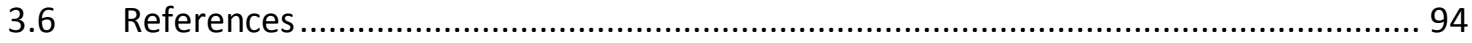

Chapter 4. Automated Quantitative Metrics in a Virtual-reality Myringotomy and Tube Simulator: Development and Construct Validity............................................................96

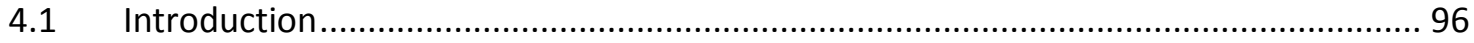

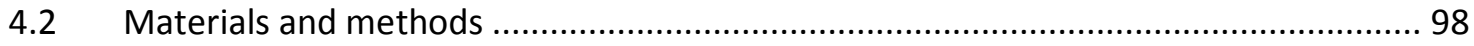




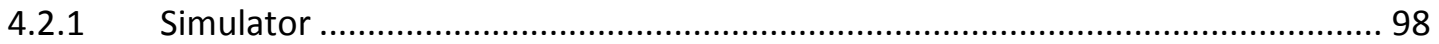

4.2.2 Development and implementation of automated metrics ................................. 99

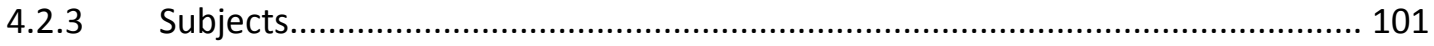

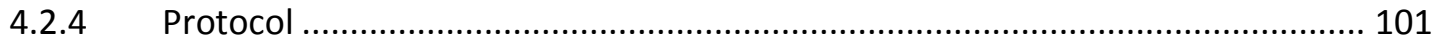

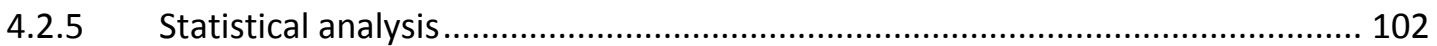

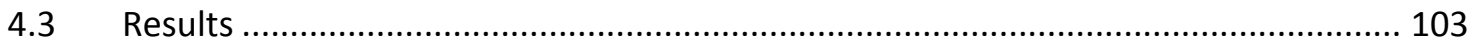

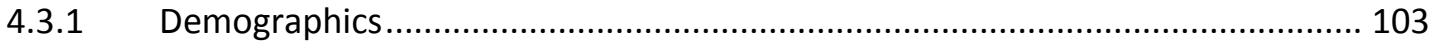

4.3.2 Automated Performance Metrics..................................................................... 103

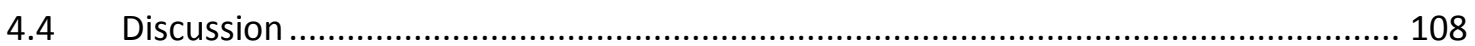

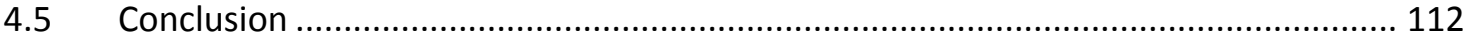

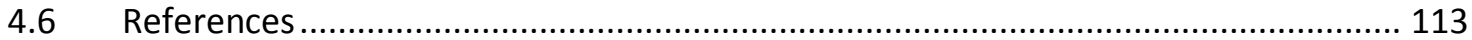

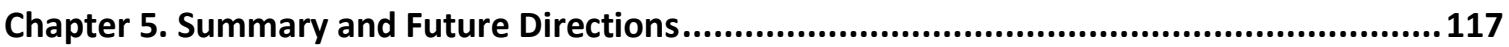

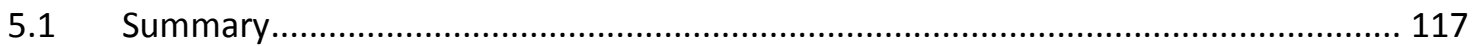

5.1.1 Simulator design and implementation ........................................................... 117

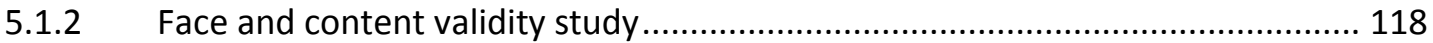

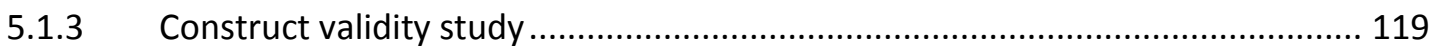

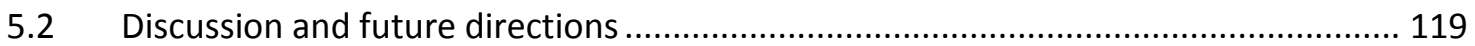

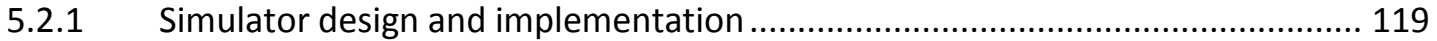

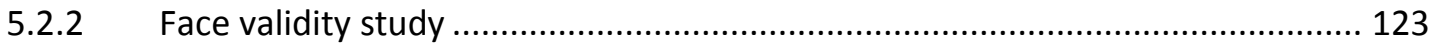

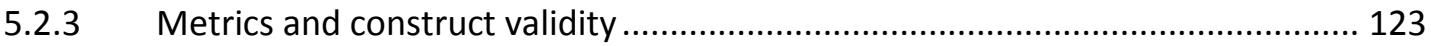

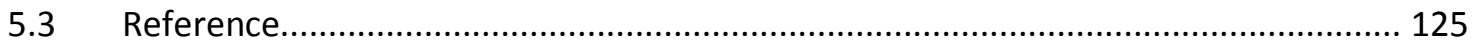

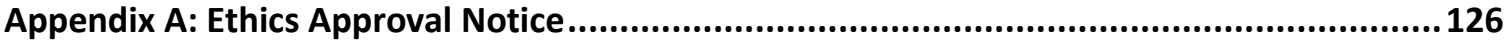

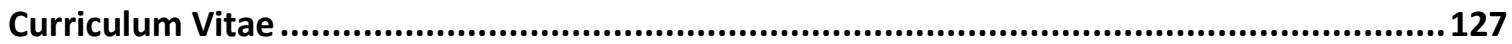




\section{List of Figures}

Figure 1.1 A longitudinal section of the human ear................................................... 3

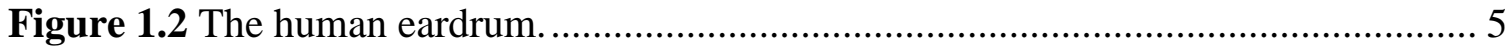

Figure 1.3 An illustration of myringotomy with tube insertion................................ 8

Figure 1.4 Surgical instruments used in Myringotomy............................................. 9

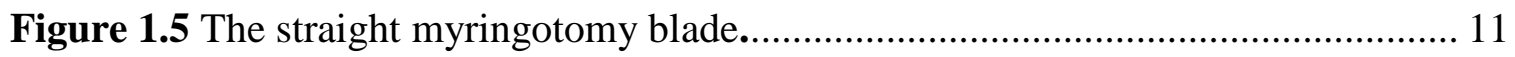

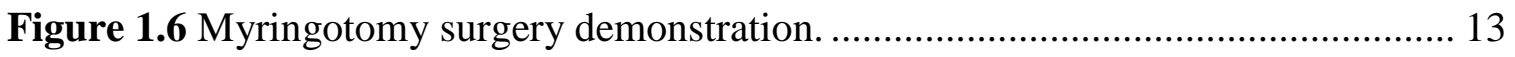

Figure 1.7 The myringotomy simulator designed by Wheeler et al............................. 20

Figure 1.8 The myringotomy simulator designed by Sowerby et al............................. 21

Figure 1.9 Three cutting algorithms implemented by Ho et al................................ 22

Figure 1.10 Examples of soft tissue surface representations using MS models. ............ 25

Figure 1.11 Sample cut with element removal algorithm........................................ 29

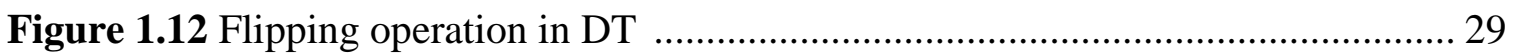

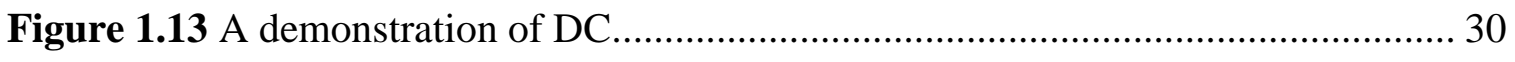

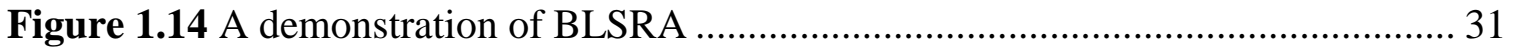

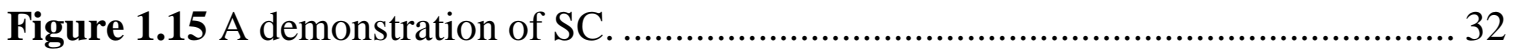

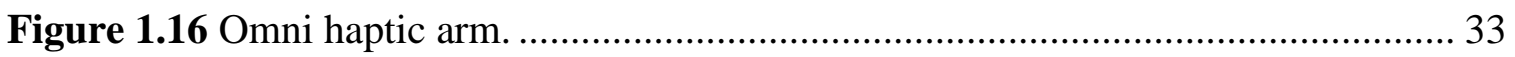

Figure 2.1 Hardware for myringotomy simulator. .................................................. 52

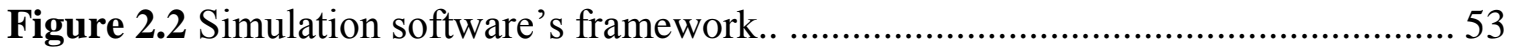

Figure 2.3 Geometrical model of the ear and speculum. ............................................ 55

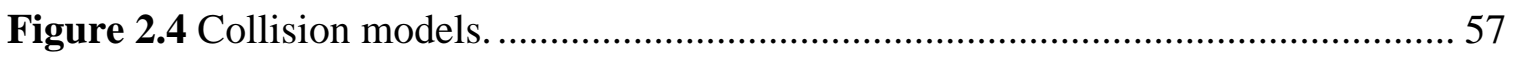

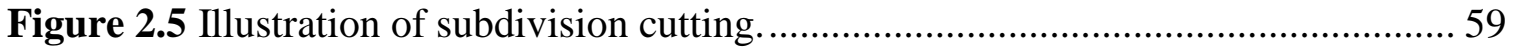

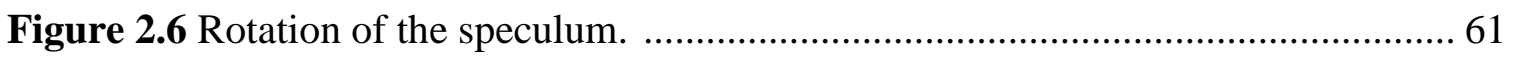

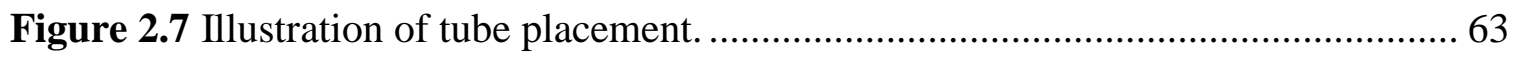

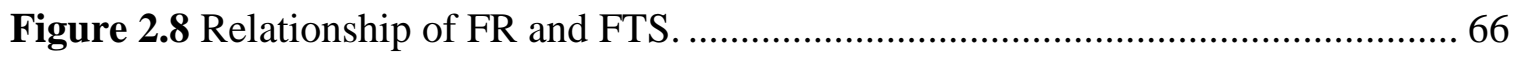

Figure 2.9 Effects of blade movement speed on cutting algorithm. ............................ 68

Figure 2.10 Simulated scene as viewed by user................................................... 70

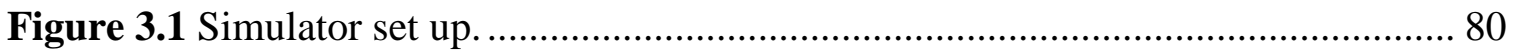

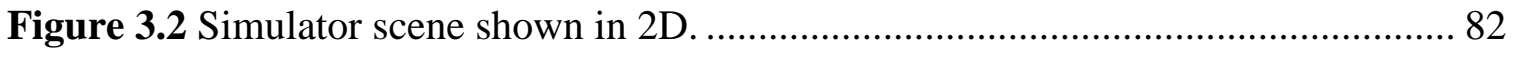

Figure 3.3 Box plot of the Likert item responses for the two groups of participants..... 87 
Figure 3.4 Total number of positive, neutral and negative responses to each question, pooling responses of junior residents and of senior Otolaryngologists.

Figure 3.5 Representation of the virtual tympanic membrane by a collection of discrete

points...... 91

Figure 4.1 Illustration of incision angle $\theta$

Figure 4.2 A two-dimensional view from the simulator is shown. .................101

Figure 4.3 Time to completion shown for each group over 10 trials..................104

Figure 4.4 Number of surgical errors shown for each group over 10 trials............105

Figure 4.5 Incision angle $\theta$ shown for each group over 10 trials................... 106

Figure 4.6 Incision length shown for each group over 10 trials....................107

Figure 4.7 Microscope magnification level shown for each group over 10 trials........108 


\section{List of Tables}

Table 1.1 Specifications of the PHANTOM Omni haptic arm .................................... 34

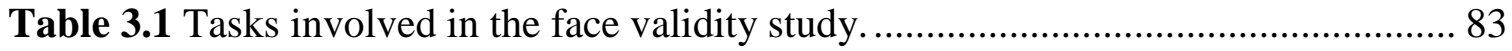

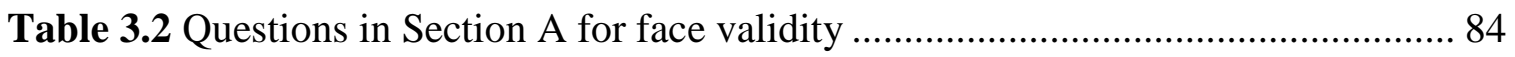

Table 3.3 Questions in Section B for training potential ........................................... 85

Table 4.1 Automated Performance Metrics Over 10 Trials..........................103 


\section{Chapter 1. \\ Introduction}

\subsection{Motivation}

In 2013, hearing loss was made a priority disease by the World Health Organization (WHO) [1]. They estimate that over 360 million people ( $>5 \%$ of the global population) have a disabling hearing loss, and that this number is expected to rise substantially in the future. The WHO states that "hearing loss is an important health concern with substantial economic costs and social consequences," and that a number of key measures are needed to help reduce the burden of the disease [1].

In children, infections of the middle ear, the portion of the ear medial to the eardrum, are common and can result in pain and a buildup of fluid within the middle ear chamber, obstructing the transmission of sound. Hearing loss can manifest without appropriate treatment of the infection. A surgical procedure (myringotomy) is usually recommended to treat middle ear infections by draining the fluid out of the middle ear and ventilating it.

The frequency of myringotomy in patients is high due to the substantial morbidity of middle ear infections in children, especially in children aged two to three years. During this age, more than $50 \%$ of Canadian children have at least one middle ear infection since birth, and the percentage with frequent middle ear infections is over 12.6\% [2]. One study in Canada indicated that $5.54 \%$ of children under 4 years old and $9.69 \%$ of children less than 11 years underwent myringotomy to treat middle ear infections [3]. According to statistics, it is the second most common surgical procedure next to circumcision for children under 15 years of age [4]. 
Myringotomy is the one of the earliest microscopic surgical procedures that residents in Otolaryngology (ear-nose-throat surgery) learn. This procedure requires high levels of manual dexterity and experience to operate surgical instruments within the human ear under a microscope. Many surgical residents have difficulties in mastering the myringotomy procedure. The clinical training of residents uses a traditional apprenticeship approach of "See one, Do one, Teach one" where patients are treated as training subjects; during this training period, there is a significant risk of damaging the delicate structures within the ear, resulting in hearing loss [5]. To help residents build their skills in myringotomy and avoid unnecessary risks to patients, a virtual reality simulator is being developed for training purposes and is described in this thesis. Following a brief review of ear anatomy and physiology, subsequent sections in this chapter review work related to simulation in order to justify the objectives of this work.

\subsection{Ear anatomy and physiology}

A brief introduction to the structure and function of the ear is provided here to aid in understanding of myringotomy. The human ear is schematically illustrated in Figure 1.1. The ear is conceptually partitioned into three anatomical regions: the outer ear, the middle ear, and the inner ear. 


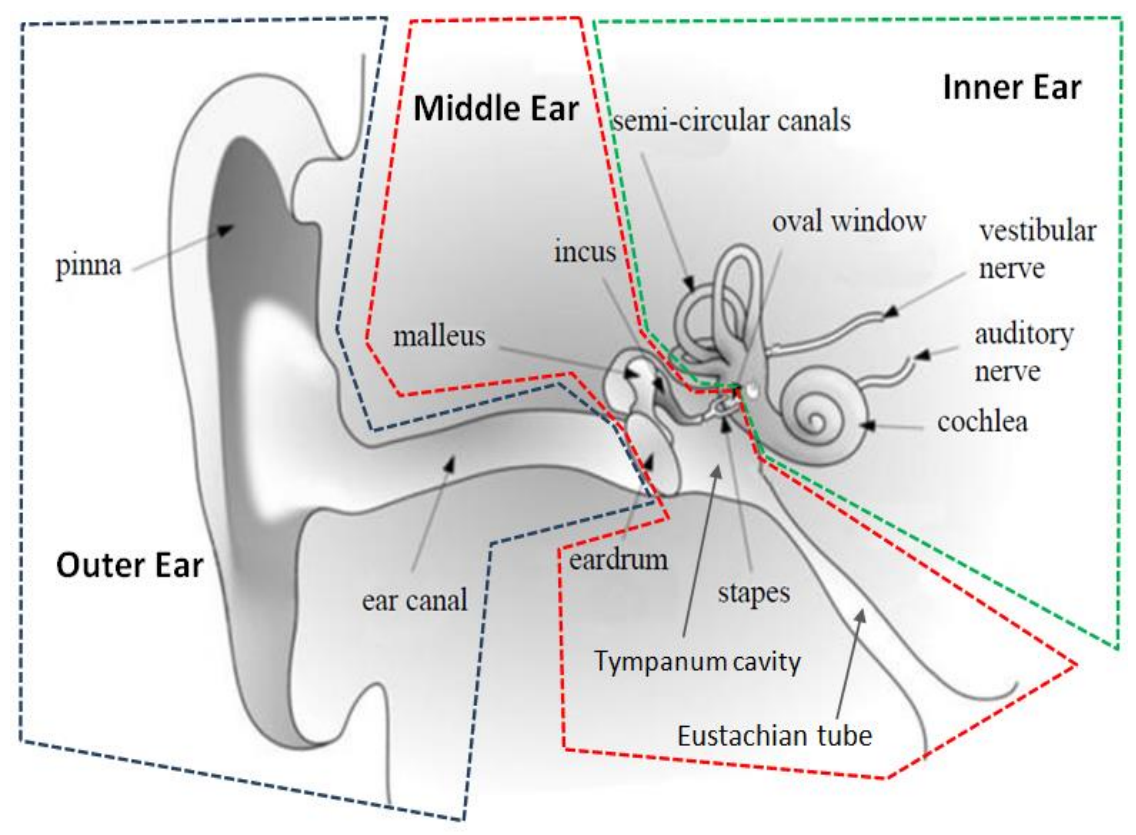

Figure 1.1 A longitudinal section of the human ear. This image was released into the public domain by its author, Dan Pickard [6].

\subsubsection{Outer ear}

The outer ear includes the pinna (auricle) and the ear canal. The pinna is the visible, projecting portion of the ear, which is composed of cartilage and skin. It is connected to the head by ligaments and muscles. Since the pinna is shaped by a cartilage frame, it can be easily deformed by surgical instruments. The form of the pinna is like a funnel with many folds that have evolved to collect sound waves, directing them into the ear canal. It preferentially amplifies sounds in the frequency range of human speech [7].

The ear canal (external auditory canal) is a curved, tube-like tunnel that runs from the end of the pinna to the eardrum. For an adult, the ear canal is about $25 \mathrm{~mm}$ in length and 7 $\mathrm{mm}$ in diameter on average [8-10]. It is made of bone and cartilage covered by skin. For an infant, the length of the ear canal is approximately $14 \mathrm{~mm}$, and its curvature is much 
smaller when compared to an adult [10]. The function of the ear canal is to amplify and transmit sounds to the eardrum [11].

In a myringotomy, a surgical speculum is placed into the ear canal and supported by the pinna. The pinna and part of the ear canal covered by the speculum can be deformed when a surgeon adjusts the speculum to check the eardrum. A surgical blade is navigated through the speculum and the remaining part of the ear canal to the eardrum.

\subsubsection{Middle ear}

The middle ear contains the eardrum (tympanic membrane) and the bones that link the eardrum to the inner ear. The eardrum is a thin, conical membrane bounding the medial end of the ear canal at an angle of approximately 43 degrees to the central canal axis [12]. It is about $0.1 \mathrm{~mm}$ thick and $90 \mathrm{~mm}$ in diameter $[12,13]$. The whole shape of the eardrum is similar to a shallow bowl that is concave slightly inwards from the head. Part of the membrane is tightly coupled with the manubrium of the malleus bone (see Figure 1.2). The eardrum vibrates in response to sound in the ear canal and sets the bones inside the middle ear in vibration. It also protects the delicate middle ear from the outside.

The eardrum can be divided into two sections: the pars flaccida and the pars tensa. The pars flaccida, as shown in Figure 1.2, is a triangular area lying above the malleolar folds. It is a relatively loose region of the membrane that is pearly white or grey color. The remaining part of the membrane is called the pars tensa. It is taut with a pinkish color. For clinical inspection, the human eardrum is usually divided into 4 quadrants: posterosuperior, postero-inferior, antero-inferior and antero-superior by drawing an imaginary 
line straight down the manubrium, then drawing a second line perpendicular to the first that runs right through the umbo - the inferior end of the manubrium.

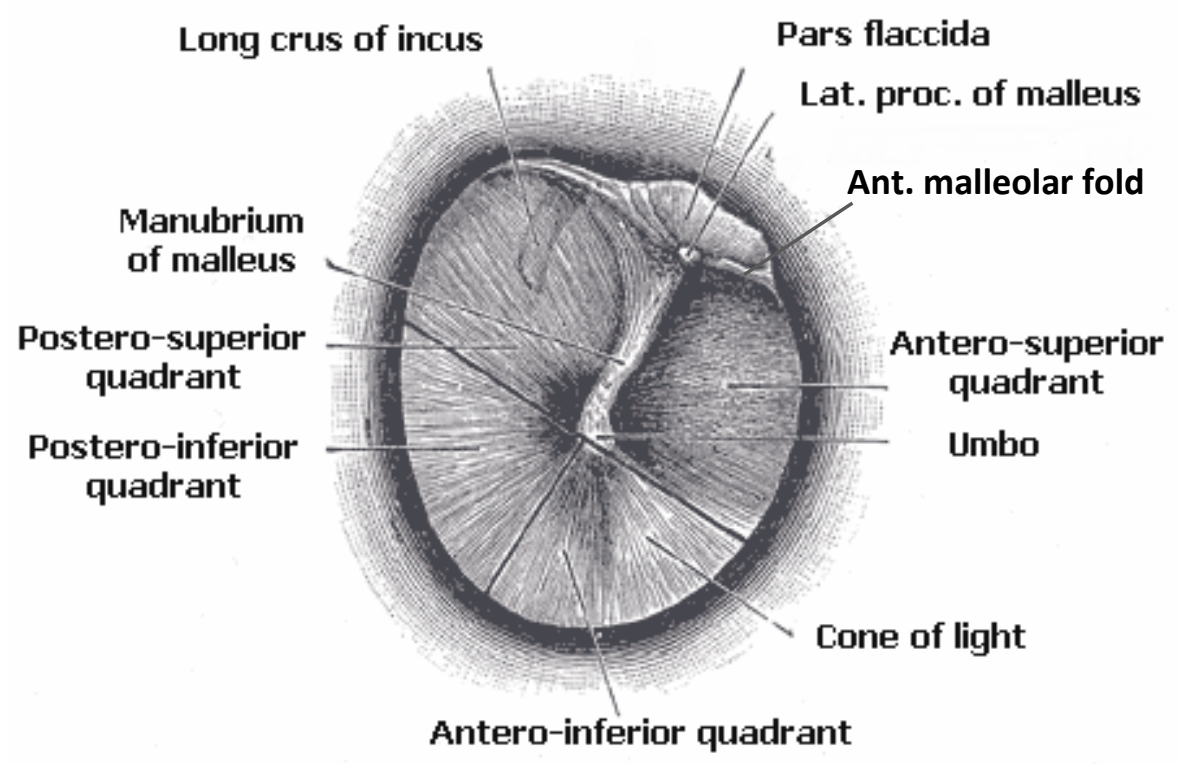

Figure 1.2 The human eardrum. This figure was reproduced from an image of Gray's Anatomy and released into the public domain [14].

The bones (ossicles) behind the eardrum include the malleus, the incus, and the stapes (see Figure 1.1). They are connected in series and cross the middle ear cavity (tympanum cavity). As previously noted, the manubrium of the malleus is attached to the eardrum. The stapes is connected to the cochlea (part of the inner ear) through the oval window. The incus is the middle bone which connects the malleus and stapes. This chain provides a leverage effect to transmit and amplify the mechanical vibrations of the eardrum on route to the cochlea. 
The middle ear bones are suspended in the middle ear cavity by ligaments and are acted upon by two muscles. This cavity is a narrow, air filled space. It is roughly $15 \mathrm{~mm}$ in width and height and $5 \mathrm{~mm}$ deep [15]. A small canal called the Eustachian tube connects the middle ear cavity to the back of the nose and upper throat. It is about $36 \mathrm{~mm}$ long [15] and allows the air into the middle ear through the upper airway from time to time by opening the mouth widely or yawning. This keeps the air pressures equal on either side of the eardrum.

Nerves (such as the chorda tympani) and arteries pass near the superior portion of the eardrum and the ossicles are also located behind this part of the eardrum; thus, surgeons prefer to make an incision in the inferior half (antero-inferior quadrant and adjacent postero-inferior quadrant) of the eardrum to avoid the vasculature, nerves, and bones associated with the eardrum during a myringotomy. When examining the eardrum with an otoscope, a landmark known as the "cone of light," will appear within the anteroinferior quadrant of the eardrum. This can help a surgeon to confirm a suitable incision site. The landmark is a reflection of otoscope light presenting at the five o'clock position in the right ear and at the seven o'clock position in the left ear.

\subsubsection{Inner ear}

The inner ear mainly includes two organs — the cochlea and the semicircular canals. The semicircular canals are part of our balance system that control one's sense of steadiness. The cochlea is directly connected to the stapes in the oval window and receives vibration signals. It is a bony structure shaped like a snail and filled with fluids. The incoming vibrations are transmitted to the membrane of the oval window and force the fluid to 
move within the cochlea. Sensory receptors in the cochlea convert the movement of the fluid to electrical impulses that are sent by the auditory nerve to the brain for processing.

In a myringotomy, the inner ear is not directly involved in the procedure. The possibility of trauma to the inner ear is low since the surgical operations focus on the eardrum and extend to the middle ear cavity.

\subsection{Myringotomy}

When a cold or middle ear infection is treated, it is common for children to have fluid remaining in the middle ear cavity since the Eustachian tube is prone to blockage by mucus during infection. Middle ear fluid often clears up without any treatment in three months. However, if the fluid remains trapped behind the eardrum for longer than three months, it may become thickened like glue and impossible to drain out naturally, even if the Eustachian tube does open up. This fluid could impede the movements of the ossicles and result in decreased transmission of sound waves from the ear canal to the cochlea. The direct consequences could be hearing loss, delay in speech development and further ear infections. In this situation, myringotomy with tube placement is recommended to prevent these negative effects.

Myringotomy with tube insertion is a surgical procedure in which a tiny incision is made in the eardrum (see Figure 1.3-a). The fluid accumulated in the middle ear cavity is suctioned out via the incision, followed by insertion of a ventilation tube into the incision, opening an airway from the outside to the middle ear similar to the Eustachian tube; this allows the newly-generated fluid to drain out continuously through the tube during the infection (see Figure 1.3-b). 

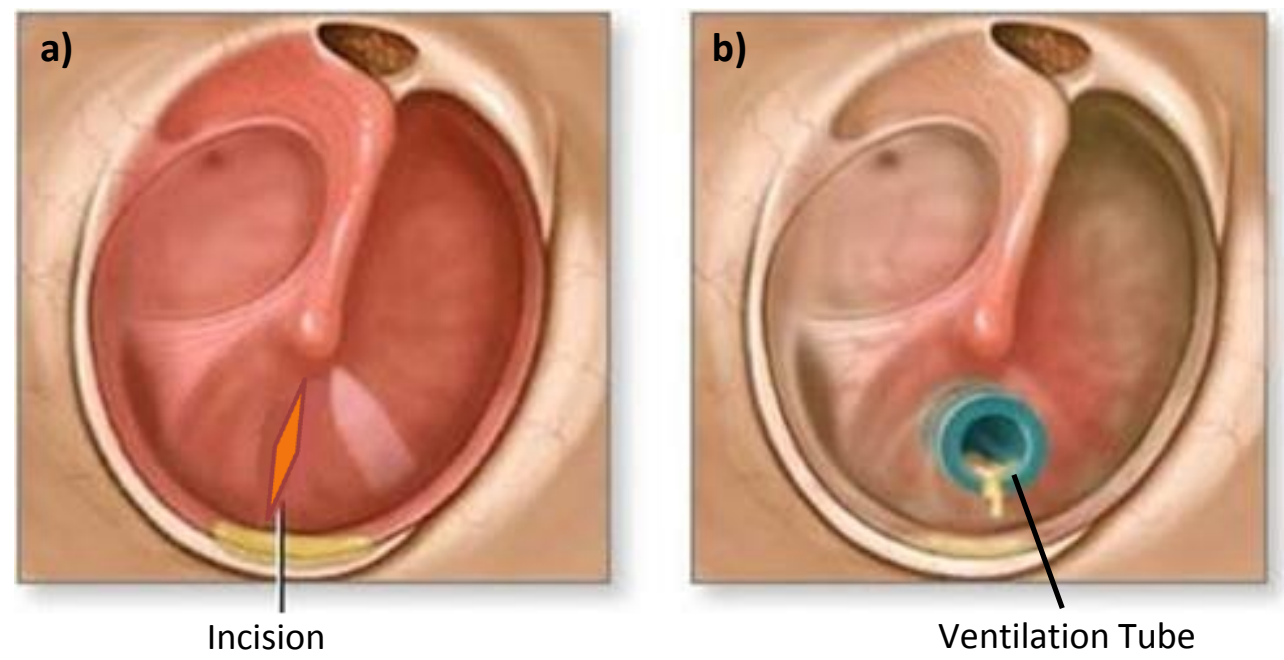

Figure 1.3 An illustration of myringotomy with tube insertion. The picture is an updated image from [16]. a) An incision is made in the eardrum. b) A tube is inserted into the incision.

\subsubsection{Surgical instruments}

The surgical instruments used in myringotomy mainly include an ear speculum, an operating microscope, a curette, a myringotomy blade, a suction tube, crocodile forceps, and a ventilation tube. The following is a brief introduction describing them. 


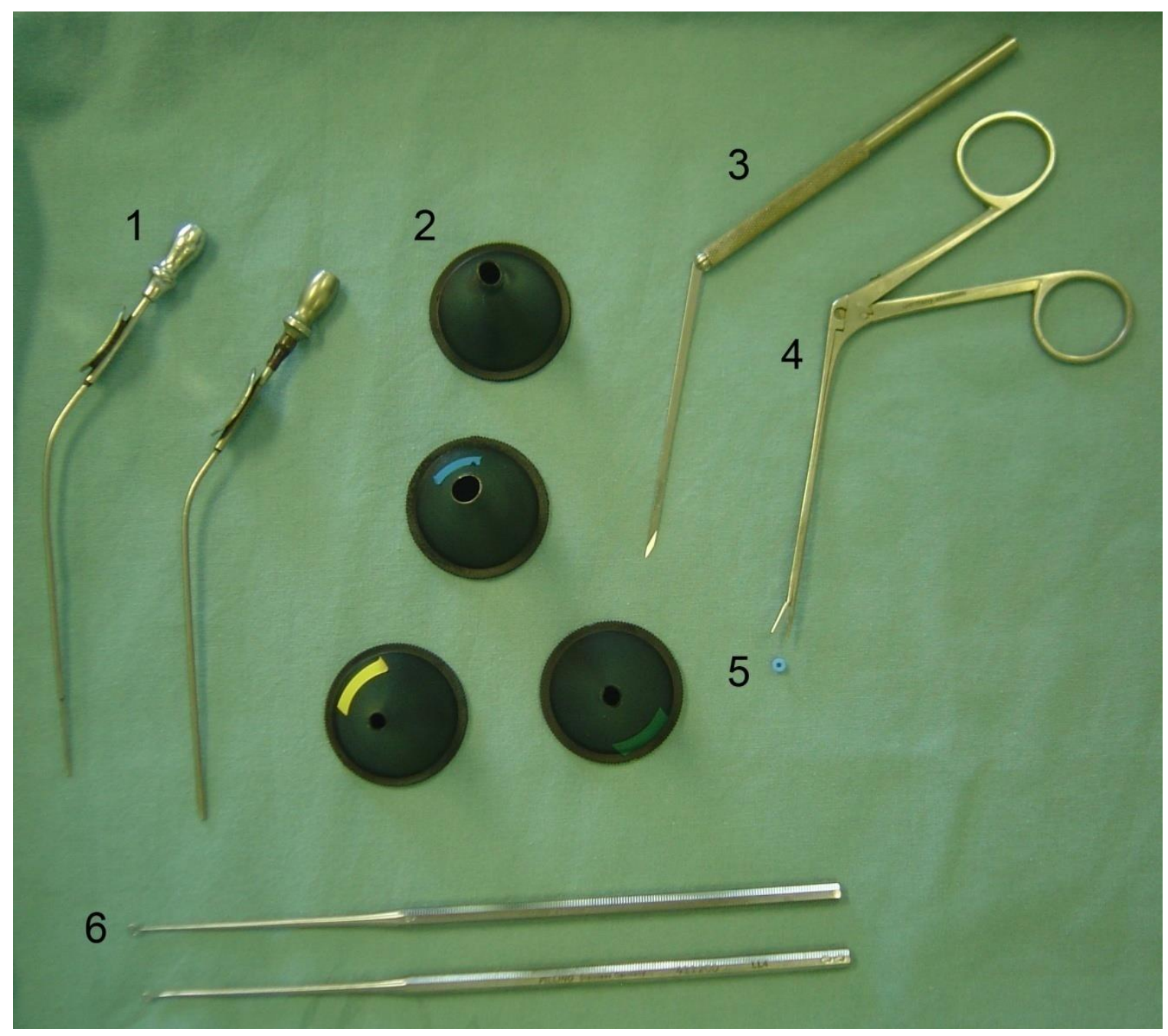

Figure 1.4 Surgical instruments used in Myringotomy. 1 suction tips, 2 specula, 3 myringotomy blade in handle, 4 crocodile forceps, 5 ventilation tube, 6 curettes.

The tools used in a myringotomy for visually targeting the cutting area on the eardrum are the speculum and a surgical microscope. The ear speculum (See Figure 1.4, item 2) is typically a short, funnel-shaped tube that is wider on one end and tapers towards the opposite end. It is made of rigid material such as stainless steel, chrome or plastic, and comes in variety of sizes from around $3 \mathrm{~mm}$ to $7 \mathrm{~mm}$. Most of them have a blackened surface to reduce light reflection. The rim of the narrow end is either round or oval. The 
oval-shaped design increases the visual axis depending on orientation. Ear speculums are used to widen the ear canal and provide exposure to the eardrum.

The operating microscope used in the myringotomy, as shown in Figure 1.6. It provides two enlarged objective images for right and left eyes for stereoscopic vision. Stereo vision gives the surgeon depth perception that helps in determining the distance between objects in a three dimensional space. The pre-determined magnification powers of a surgical microscope used in myringotomy are minimally $10 \mathrm{x}$ or $12.5 \mathrm{x}$, and further manual fine focusing is also available. The working distance from the microscope objective lens to the point of focus is about $200 \mathrm{~mm}$. The microscope's illuminator emits a coaxial light that penetrates even the narrowest cavities, illuminating the field of view brightly and uniformly. During the procedure, the surgeon can adjust the position, focus, zoom and illumination of the microscope.

Before cutting the eardrum, the surgeon often uses a curette to clean the ear canal. The ear curette is a long, straight or curved tool designed for removing wax and debris from the ear canal. The tip of the curette is a small scoop or a circular ring as shown in Figure 1.4 (item 6).

A myringotomy blade is the tool used for cutting the eardrum. It is usually an angled blade made of stainless steel (see Figure 1.4, item 3). The narrow blade shaft is mounted at 45 degrees to the handle. This permits the surgeon to hold the blade's handle without blocking the line of sight through the microscope. The tip of the blade can be double edged to perform bidirectional cutting, but typically a single edged blade is used for 
unidirectional cutting. The straight blade (see Figure 0.5) is another commonly used cutting tool for myringotomy.

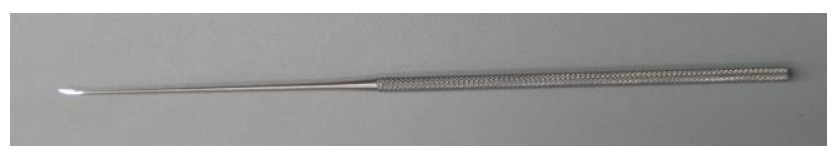

Figure 1.5 The straight myringotomy blade.

A suction device is used to remove the fluid from behind the eardrum. It is composed of a slender suction tube (see Figure 1.4, item 1) and a vacuum aspirator. The suction tube is about $3 \mathrm{~mm}$ in diameter, $200 \mathrm{~mm}$ in length, and is bent at an angle. The handle is connected to the vacuum aspirator.

When inserting a ventilation tube, a pair of crocodile forceps (see Figure 1.4, item 4) is used to pick up the tube and insert it into the incision. The forceps have small jaws at the tip, connected to handles by a long, sliding linkage that allows the jaws to be passed through a narrow canal and still operate without impediment. By squeezing the handles, the distal jaws close together to grasp a small object between them and reversing the action of the handles releases the jaws. The handles have an offset angle to the linkage neck to avoid obscuring the visual field during the operation.

The ventilation tube comes in a variety of designs. The most commonly used type is shaped like a grommet, made of plastics such as silicone and Teflon. There are two flanges at both ends of the tube. They are connected to a cylindrical shank at an angle of $90^{\circ}$ to the axis of the shank. The diameters of the flanges can range from 1.5 to $3.0 \mathrm{~mm}$. The inner diameter of the cylindrical shank is usually 0.76 to $1.27 \mathrm{~mm}$. The length between two flanges of the tube is about 1 to $2 \mathrm{~mm}$ (see Figure 1.4, item 5). 


\subsubsection{Tasks in the myringotomy procedure}

Before a myringotomy, general anesthesia is administered by a mask. The patient is placed in a supine position on an operating table. The patient's head is supported on a doughnut headrest and turned slightly so that the affected ear is up and leans towards the surgeon who will perform the surgery. The surgeon sits while operating as shown in Figure 1.6. An operative microscope is adjusted by the surgeon to obtain a clear view of the infected ear. A speculum is placed into the ear canal and the surgeon uses one hand to hold the wider end of the speculum while keeping the narrower portion in the ear canal. A view of the eardrum is obtained and seen through the narrow end of the speculum and magnified by the microscope. The surgical view can be adjusted by rotating the speculum and tilting the microscope accordingly. If there is wax accumulation, the surgeon will remove it using a curette until the entire eardrum can be visualized. Then the blade is navigated through the speculum and ear canal, and a tiny incision is made in the eardrum. After suction of the middle ear fluid via the incision, a ventilation tube is directed towards the incision using crocodile forceps. One flange of the grommet tube is placed in the incision, and then the tube is pushed and rotated slightly into the incision. If the incision length is not adequate, a second cut can be performed to enlarge the incision. Finally, a needle is used to adjust the tube to ensure it is sitting in the proper position in the eardrum. The whole procedure can be completed within three minutes. 


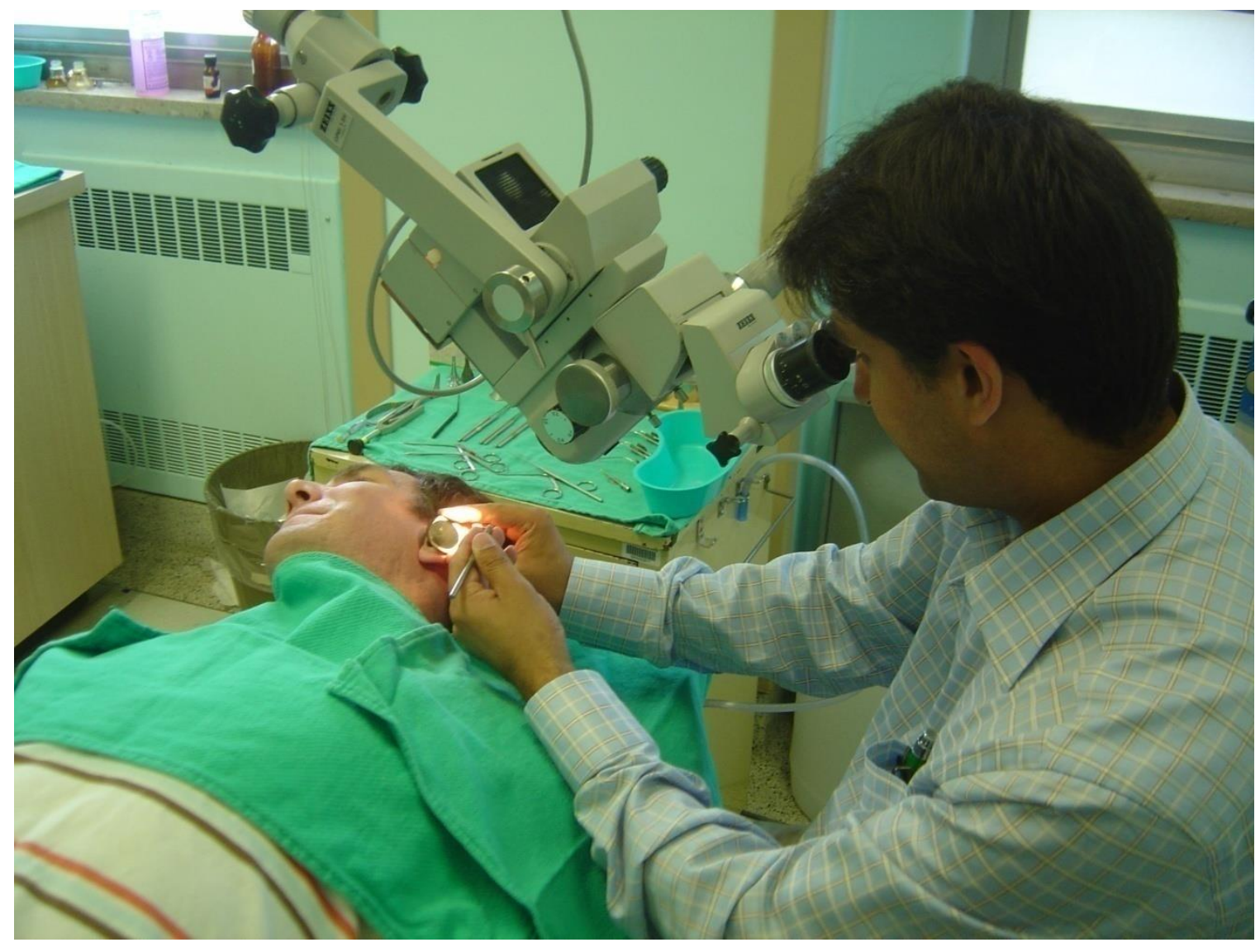

Figure 1.6 Myringotomy surgery demonstration. A surgeon places a speculum into the left ear of the patient using the right hand while the left hand holds a myringotomy blade. A microscope is used to observe the inside of the ear exposed by the speculum.

\subsubsection{Technical requirements and errors}

The whole process of a myringotomy procedure is performed under a microscope. During the procedure, high levels of dexterity and experience are needed to complete all operations in a very short time period. The critical skills include tilting the microscope and zooming to obtain an appropriate view, selection of an appropriately sized speculum adapted to the depth and diameter of the patient's ear, navigating the blade down to the eardrum without cutting the ear canal, cutting at the recommended site - the inferior half of the eardrum to avoid the vasculature, nerves, and bones associated with the 
eardrum, cutting in the recommended direction — radially from the start point moving outward along the radius centered at the umbo, and cutting to an appropriate length (approximately 2 to $3 \mathrm{~mm}$ [17]) adequate for the size of the ventilation tube allowing it to be inserted and seated in the incision. Finally, the cutting blade should not go too deep into the middle ear cavity to prevent damaging the ossicles behind the eardrum.

Twelve potential error types made by surgical residents were tabulated by Montague et al. [18]. The four most frequent errors identified were (1) failure to perform a unidirectional myringotomy incision (43\%); (2) multiple attempts to place ventilation tube instead of one attempt (16\%); (3) multiple attempts to complete the myringotomy as opposed to one attempt (13\%); and (4) using a magnification setting that was too high (13\%). Others were using a speculum that was too large or too small, traumatic clearance of wax, the wrong myringotomy site, a circumferential incision, making a myringotomy incision too large or too small, and failing to aspirate the middle ear fluid. Furthermore, experience at Western University suggests that the number of accidental contacts of the blade with the ear canal and with the middle ear bones can also be considered errors in blade navigation and incision creation.

\subsection{Training in myringotomy}

Currently, surgical residents are taught and trained in the procedure using either actual patients in the clinic or operating room or using cadavers in the laboratory.

The standard apprenticeship model (Halstedian approach), as used in other surgical specialties [19], is also employed for training in myringotomy. Residents learn about the surgery, watch experienced surgeons performing the surgery and perform the surgery 
themselves. Through a monitor connected to the microscope, views of surgical scenes are displayed on a screen in real time. The residents can observe all the steps of an entire surgery demonstrated by an experienced surgeon. After assisting in a sufficient number of surgeries, the residents are allowed to perform the surgery on a real patient under the supervision and assistance of an expert surgeon. The major drawback of this approach is the potential risk of trauma to the patient. Moreover, the opportunities to practice inside of the operating room are limited by the number of available patients who present during a trainee's residency.

Training on cadavers is another way to improve the skills of trainees. However, because cadaver donations are becoming increasingly scarce and cadaver preservation costs are high, their use for training a large number of residents is unsuitable. Furthermore, an expert is still required in supervising the trainee's progress during this training.

Physical models such as the Wigan Grommet Trainer [20], the Bradford Grommet Trainer [21], and artificial ears [22-23] are designed to provide practice in myringotomy without the risk of harm to patients. In these simple models, a rigid tube is typically used to represent the ear canal, and a flat synthetic membrane is affixed to one end of the tube to represent the eardrum. A further development of such a model was reported in [24]. The component of the ear canal was made of acrylonitrile butadiene styrene (ABS) plastic and was bent $10 \%$ in the medial third to simulate the anterior bony overhang. These replicas are placed under a surgical microscope and operated upon by residents. Physical replicas do not accurately represent the geometry and mechanical properties of the ear canal and eardrum, nor can they easily simulate inter-patient variability. In 
addition, there is no method for providing automatic feedback to the trainee. Thus, physical replicas are not commonly used in training.

Surgical simulators based on virtual-reality (VR) technologies have been introduced as training tools to overcome the shortcomings of the apprenticeship approach and of physical models. A VR system is a computer-based application that provides real-time interaction with digital models of deformable tissues by pushing, cutting, suturing, and even drilling. VR-based simulators can provide realistic visual scenery and force feedback through special hardware interfaces. The geometries and mechanical properties of virtual objects such as tissue models can be changed easily. The user's actions can be recorded and analyzed to provide quantitative feedback on surgical technique and skill progression. Ideally, it can be a self-contained teaching tool.

The first VR-based myringotomy simulator was created by the Auditory Biophysics Laboratory (ABL) at Western University [25-27] (see section 1.5.2). Various aspects of the myringotomy procedure are implemented as separate systems. One is a training system for surgical tool navigation $[25,26]$, in which a trainee can see a 3D model of the ear through a stereoscopic visor, and navigate the surgical blade through the ear canal to make an incision on the eardrum by drawing a line on it. Another one is a cutting simulation system [27], in which a user can perform an incision on a deformable eardrum while force feedback can be applied to the user's hand via the haptic device to give the sense of cutting through tissue.

The current simulator cannot be used to perform ventilation tube insertion into the virtual incision. From discussions with surgeons at Western University and elsewhere [18], it is 
apparent that tube insertion is challenging for most residents to master. In addition, the simulator does not provide quantitative feedback on the skill of the trainees.

\subsection{Literature review}

\subsubsection{Simulators in Otolaryngology}

In Otolaryngology, VR surgical simulators have been developed as training tools [28-29], especially for endoscopic sinus surgery [30-37] and temporal bone drilling [38-42].

In sinus surgery simulation, the Lockheed Martin endoscope sinus surgery simulator (ES3) [30] was the first and leading simulator used for training in the past ten years. It ran on a Silicon Graphics workstation. The surgical view could be adjusted by manipulating an endoscope replica. Force feedback was transmitted through a surgical handle replica. Both the endoscope and surgical handle replicas were set in a rubber head model when performing the simulation. Four validation studies covering face, content and construct validity, were completed between 1998 and 2010 [29]. The validity studies demonstrated that the ES3 was a very good simulator, but its antiquated computer platform cannot compare with current high-end computer systems. The ES3 simulator is no longer being made. Another two notable simulators were VOXEL-MAN SinuSurg [33] from the University of Hamburg - Eppendorf group in Germany and the McGill simulator for endoscopic surgery (MSESS) [34]. The SinuSurg ran on a standard personal computer. A Phantom Omni haptic arm (Geomagic, Inc., Morrisville, NC) was employed for the positioning of surgical tools and adjusting of microscope and endoscope and providing force feedback. The user could also see a stereoscopic surgical view through shutter glasses. However, feedback from users has yet to be collected. The MSESS was 
developed on the NeuroTouch platform $[35,36]$, which was a neurosurgery simulator made by the National Research Council of Canada. It was equipped with two haptic arms for positioning and providing tactile feedback. A 0-degree endoscope and a microdebrider were connected to the two haptic arms respectively permitting the user to practice holding an endoscope with the non-dominant hand and removing tissue using a microdebrider with the dominant hand. Two pedals were designed for the control of washing the endoscope and activating the microdebrider. The surgical scene was displayed on a flat panel monitor. A validity study demonstrated its realism in terms of appearance and its capacity for differentiating different levels of expertise [37].

In temporal bone surgery simulation, the simulators at Stanford University [38-39], the Ohio State University [40-41] and VOXEL-MAN TempoSurg [42-43] are well known VR-based simulators. TempoSurg was the most studied and first commercialized temporal bone simulator. It ran on a high-performance personal computer with a Windows platform. Two PHANTOM Omni haptic arms were employed to enable twohanded operations including drilling, suction, and bleeding. A foot pedal was utilized to control the drilling speeds of burs of different shapes. Drill and suction sounds were rendered depending on drill pressure and speed. The unique volume sculpting algorithm contributed to a highly detailed visual and haptic rendering when drilling and milling of hard tissue such as bones and teeth. A virtual stereoscopic scene could also be experienced through a pair of 3D goggles. Automatic scoring of the trainee was provided during the operation [42]. Face, content and construct validity were thoroughly tested by 6 studies from 2007 to 2012 [43]. The Ohio State University's simulator had a similar interface to the VOXEL-MAN TempoSurg including a 3D stereoscopic visual device, a 
haptic component (e.g., Omni haptic arm), a pedal and an aural element. An intelligent tutor was developed to highlight relevant anatomic structures within the temporal bone upon the user's prompt, and automatic feedback was provided to assess the trainee's performance through validated metrics [40]. A multi-institutional study indicated that there was no difference in training performance between two groups of learners, one group using cadaveric temporal bones and the other using the virtual simulator [41]. The simulator at Stanford University was the first temporal bone simulator to be developed. The system used a volumetric voxel model for rapid collision detection and bone removal, while a surface-based model was used for visual rendering. A haptic tutor was designed to allow the trainee to feel forces experienced by a remote expert operating on the same bone model [38]. The instant automatic feedback was designed to promote trainee selflearning on the simulator. A construct validity study was completed by performing a mastoidectomy twice by two groups: eight experts and seven novices [39].

\subsubsection{Simulators in myringotomy}

For myringotomy, several aspects of VR-based myringotomy simulation were implemented by the Auditory Biophysics Laboratory at Western University. First, Wheeler et al. [25] created a training module for blade navigation as shown in Figure 1.7a). An idealized 3D graphical model of an ear was created. The ear canal was modeled as a straight and hollow cylinder. The eardrum was represented as a flat circular plate attached to the inner end of the ear canal. A virtual speculum was embedded in the open end of the ear canal. A mock surgical microscope consisting of a visor for stereoscopic display and a customized stand was used to visualize the model. A real blade was tracked in 3D space by a Claron Micron Tracker (Claron Technology Inc., Toronto, Canada), and 
collisions between the blade's virtual counterpart and the virtual ear canal were detected. The incision in the virtual eardrum was represented by a straight line drawn from the point where the virtual blade first touched the eardrum to the last touching point (Figure 1.7-b). The Object-Oriented Graphics Rendering Engine (OGRE) [44] and the Open Dynamics Engine (ODE) [45] were employed as software tools for graphic rendering and collision detection. A face validity study was performed by four residents and two practicing surgeons. The results suggested that the simulator may be useful for improving hand-eye coordination and motor skills related to the myringotomy procedure. Since no haptic device was included in the hardware setup, this simulation was not able to provide tactile feedback. Also, due to limited tracking accuracy, the tracking system created too much tool vibration displayed in the virtual scene whenever the tracked tool (blade) was in motion or static.
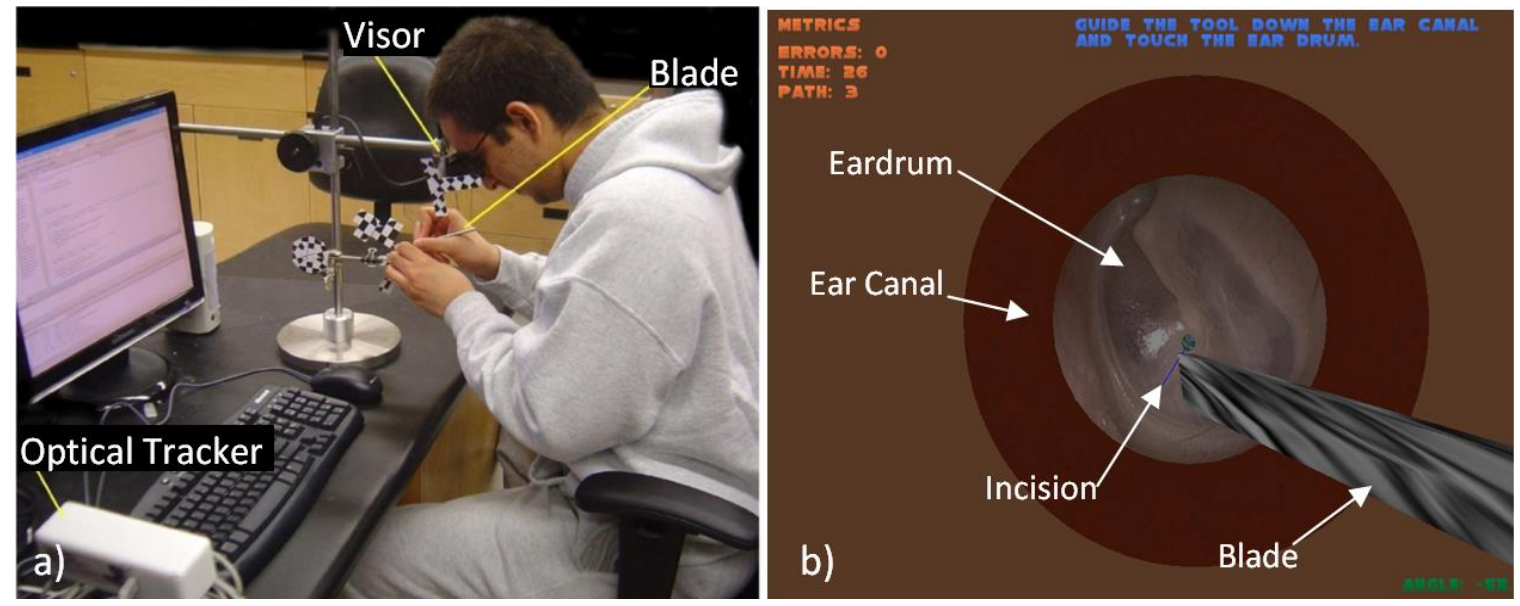

Figure 1.7 The myringotomy simulator designed by Wheeler et al. [25]. a) A mounted three-dimensional visor was used to simulate binocular vision of the microscope. The blade was tracked by an optical tracker. b) Snapshot of scene through the visor when performing the myringotomy incision. The incision was simulated by drawing a straight line on the eardrum. 
Second, Sowerby et al. [26] replaced the optical tracker with a PHANTOM Omni haptic arm. The simulator setup is shown in Figure 1.8-a). OpenHaptics [46] and OGRE were used as the software development platforms. The incision was simulated by simply drawing a curve on the surface of the eardrum using the virtual blade (Figure 1.8-b). Force feedback was programmed for the situation when the virtual blade touched the ear canal and eardrum and the level of force feedback was adjusted empirically by two experienced surgeons. It was demonstrated that the minimal force that can be rendered by the employed Omni haptic arm was larger than the force perceived by a surgeon when cutting the eardrum. Even so, receiving force feedback from Omni when the virtual blade was touching a bony structure such as the ear canal was valuable. Seven residents and four Otolaryngologists demonstrated face validity of the simulation [26].
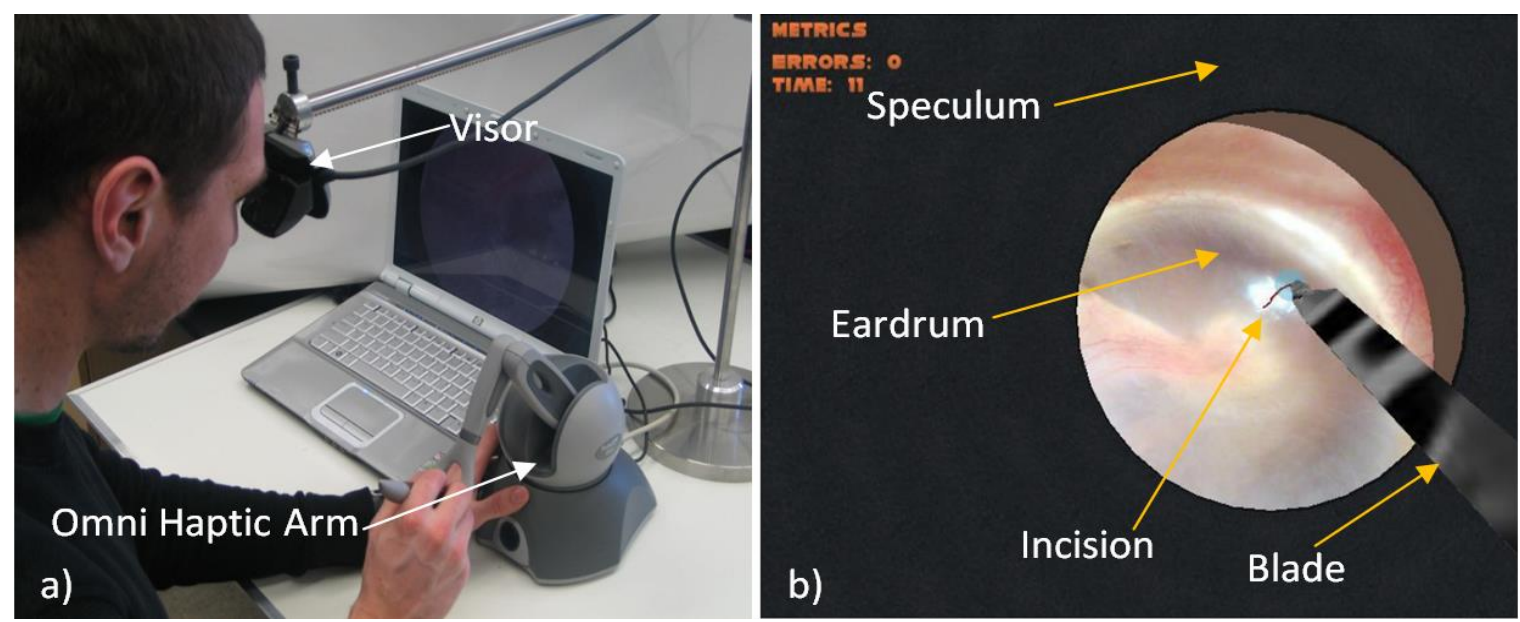

Figure 1.8 The myringotomy simulator designed by Sowerby et al. [26]. a) An Omni haptic arm was employed for the surgical tools' positioning and providing force feedback. b) A screenshot when performing a myringotomy incision. The incision was represented by drawing a curve on the eardrum surface by the blade.

Third, since there is no support for deformation modeling in OGRE and ODE, Ho et al. [27] implemented a deformable eardrum with cutting capabilities using the CHAI3D [47] 
platform. A topologically correct eardrum was modeled using the mass-spring method, and cutting was implemented using the element removal, direction prediction and Delaunay cutting algorithms, as shown in Figure 1.9. The performance of the three cutting algorithms running on the deformable eardrum was tested by eight surgeons and four surgical residents. The results were summarized by a face validity study. Overall, the Delaunay cutting algorithm was perceived to be more realistic than both element removal and direction prediction methods. However, in this simulation, the speculum, microscope and ear canal were not implemented in the myringotomy simulation.
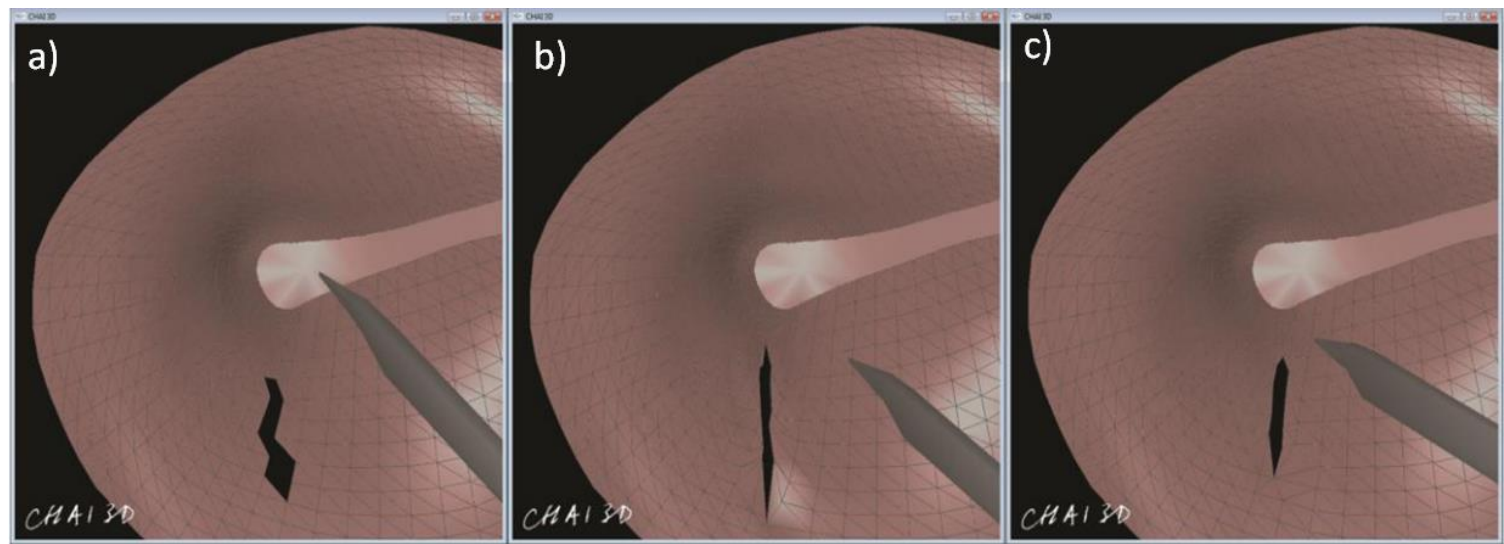

Figure 1.9 Three cutting algorithms implemented by Ho et al. [27]. a) element removal, b) direction prediction, c) Delaunay cutting.

Since the simulator was fragmented into different functional modules running on three incompatible software platforms, and the operations of the speculum and microscope as well as tube insertion were not simulated by these modules, trainees were unable to practice the myringotomy procedure with tube insertion as a whole. An integration of these processes into one single system is essential to produce an end-to-end simulator for surgical residents. 


\subsubsection{VR algorithms and hardware}

VR-based surgical simulation pursues immersive interactions between users and virtual tissues, which could be interpreted by real-time visual rendering, realistic deformation of the tissues and tactile feedback. A simulation system is, in essence, a harmonious unification of these effects. The underlying approaches to achieve these effects, such as mesh rendering, tissue deformation modeling, cutting simulation, collision detection, and force feedback calculation can dramatically impact the performance of the system due to computational loading, and therefore impact the overall effect of the simulation. Each of these effects may have a variety of algorithmic solutions with different computational complexities. The selections of these algorithmic solutions are subject to the level of rendering speed and precision required in a specific simulation. In order to achieve a good simulation effect, choosing a suitable combination of these algorithmic solutions is critical.

Moreover, the software platform and the hardware used for the implementation also should be well selected according to the functions to be realized in the simulation. The selection of a software platform pertains to the required algorithmic solutions. As hardware, for example, a haptic device should be selected for rendering the force feedback in the simulation of cutting tissue. A computer is an essential part of a VR simulation system for all the calculation tasks and control. In practice, the calculation capacity of a computer's processor is limited; there is often a trade-off between the accuracy, which can be set in the software solution, and the computational loading, which is limited by the hardware, for each of the tasks. So, it is necessary to customize both the hardware and software platforms to fit the implementation of the specific simulation tasks. 
Furthermore, as part of the simulator design, the validation of the implemented simulator is essential. The design of the validation methods need not only stem from the nature of scientific validation, but also should apply to the specific research domain — virtual reality surgical simulation.

Therefore, prior to designing a simulator, it is important to investigate the possible algorithmic solutions, hardware and software platforms and validation method. The following sections review these topics as they relate to Otolaryngology, particularly to myringotomy with tube insertion.

\subsubsection{Deformation modeling}

Deformation of soft tissues needs to be visually realistic and needs to be performed in real time for interactive performance. For training applications, simulated deformations do not need to be as accurate as in predictive models used for surgical planning, and the acceptability of deformation simulation can be judged through a face validity study, as for instance in [27]. Most deformation modeling uses physics-based methods, which calculate deformation according to structural mechanics. The mass spring method and finite element method are two numerical approaches that are commonly used to simulate tissue deformation.

\section{Mass spring method}

The mass spring (MS) method models the object as a spatial distribution of point masses that are connected to each other with springs and dampers (Figure 1.10). Each point is described by its position, mass, velocity and acceleration. 


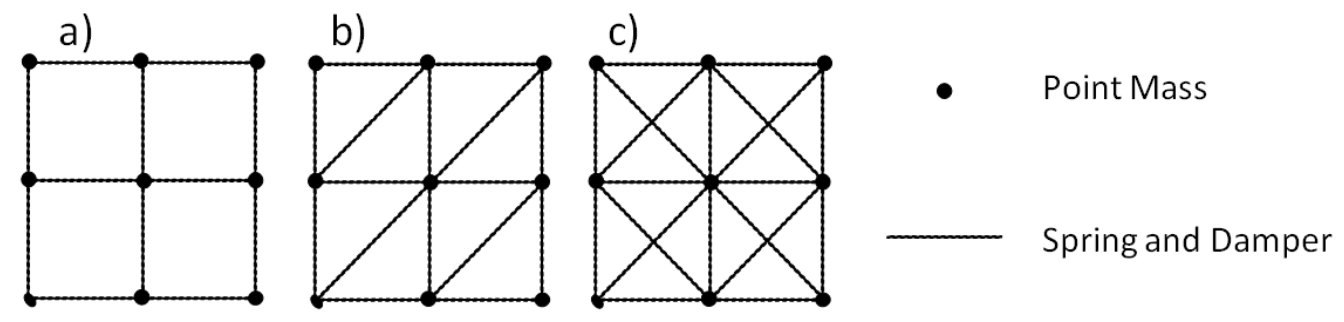

Figure 1.10 Examples of soft tissue surface representations using MS models. Several topologies are used with MS models including a) quadrilaterals, b) triangles, and c) duplicated triangular shapes.

As early as 1992, the MS method was used to model nonuniform 3D surfaces such as the human head and foot [48]. In 1999, the MS method was extended to simulate collisions, carving, cutting, tearing, and joining for use in surgical training [49]. Brown et al. introduced a quasi-static algorithm as opposed to a fully dynamic algorithm to speed up computation time. Their application was used in microsurgery simulation [50]. The algorithm was based on the assumption that the tissue's deformation occurs at a very slow speed and is well damped, thus allowing inertial effects to be ignored. Other researchers have improved the MS method including optimization of the distribution of points to better model the geometry and response [51-54], optimal selection of parameters [55] and combining the method with a gas filled model simulating a tissue's internal pressure [56], and limiting computations to small regions to speed up computation [57].

The MS method has obvious advantages, such as less expensive computation than the finite element method and ease of handling changes in mesh topology because of surgical operations such as cutting. The main drawbacks of the MS method are potential for oscillatory or unstable behavior and limited accuracy. 
Since the eardrum is a fibrous surface, the MS method is highly suitable for simulation of eardrum deformation and cutting.

\section{Finite element method}

The finite element (FE) method handles complex geometries by dividing the geometry into simple surface (e.g., triangles) or volumetric elements (e.g., tetrahedra). The simpler elements are easy to analyze and analysis can be done automatically by computers, resulting in an element stiffness matrix relating nodal displacements and rotations to

applied nodal forces. The solutions to each individual element are then combined into a structural stiffness matrix that can be inverted to compute the organ's deformation. Since the FE method is based on continuum solid mechanics, it can potentially provide accurate deformation results if the modeling parameters are set appropriately. Accurate deformation leads to visual realism.

The main disadvantage of the FE method is that it is computationally expensive to use in real-time simulation. Also, when simulating a procedure such as cutting, it is hard to change the FE mesh structure in real time because element stiffness matrices will need to be recalculated and reassembled [58]. Many researchers have tried to overcome these drawbacks, and adjust the FE method to meet the requirements of real-time surgical simulation [59-62].

\section{Other deformation models}

Other modeling methods include the Tensor-Mass Model (TMM), Finite-Spheres Model (FSM), Long-Element Model (LEM), ChainMail, and Neural Network Model which are described briefly below. 
- TMM discretizes the object into tetrahedra. The edges of the tetrahedra are the tensors. The nodes of each tetrahedron store the lumped mass of the object. The calculation method of TMM is derived from the FE method and the computations are independent of mesh topology [63]. Because of the simplified structure of TMMs, the calculation time of a TMM is less than the FE method and maintains the accuracy of the FE method. TMMs can be applied to simulate tissue cutting and tearing. Delingette et al. developed the TMM as a continuum model based on linear elasticity $[63,64]$.

- FSM is a meshless approach. It represents objects as a collection of nodes like MSM, but there is no direct link between the nodes. The nodes possess a finite region of influence which determines their effects on neighbouring nodes and coordinates their motions during simulation. Since no mesh is used, a multi-resolution strategy can be applied to zoom into regions of interest with no expensive computations. Also, since there is no mesh to constrain the movement of the nodes, they can slide past each other. This makes FSM suitable for simulating phenomena such as water, fog, as well surgical cutting [65].

- LEM supports static or dynamic solutions for elastic and plastic deformations of biological tissues, which are treated approximately as objects filled with incompressible fluid. It discretizes the volume of an object into a set of long elements. Each element starts at a point of the surface bounding the object and crosses the volume until it reaches the end of the material. The deformation is calculated based on Hooke's law and Pascal's principle [66]. It is suitable for simulation of small topological changes such as cutting $[67,68]$. 
- ChainMail is a non-physics based method. The elements of an object are simulated by a linked chain. The movements of the elements are calculated by arbitrarily displacing an element contacted by a tool and then propagating the displacement under certain constraints as in a real chainmail. Fast computational speed is the main advantage of the ChainMail method. However, it is difficult to adjust the constraints of the model to represent the physical characteristics of a given material. An extension of ChainMail has been developed to support web-based surgical training [69].

- Neural networks are used by researchers to learn the relationship between the applied force and the resulting displacement depending on the test data used to create the models. As an example, this method has been applied to a cataract eye surgery simulator to model the deformation of eye tissues [70]. Another example is using a fuzzy-neural network combined with a physics model to create a simulation system [71].

\subsubsection{Cutting methods}

As noted earlier, the eardrum is a membrane; therefore, surface cutting methods as opposed to volumetric cutting methods are suitable for simulation of eardrum cutting. Here, an "actual" cutting curve is defined as the path traced by the user, whereas the "sampled" cutting curve is a polyline representation of the actual cutting curve. A very simple virtual surface-cutting operation can be implemented by directly removing the mesh elements that are intersected by the actual cutting curve (see Figure 1.11). More realistic methods involve re-meshing of the intersected meshes. The re-meshing method reconstructs the boundaries along the cutting curve to get a smooth visual representation. 
The reconstructed boundaries form the sampled cutting curve. Three major kinds of implementations of re-meshing based cutting can be found in the literature and are briefly described below.
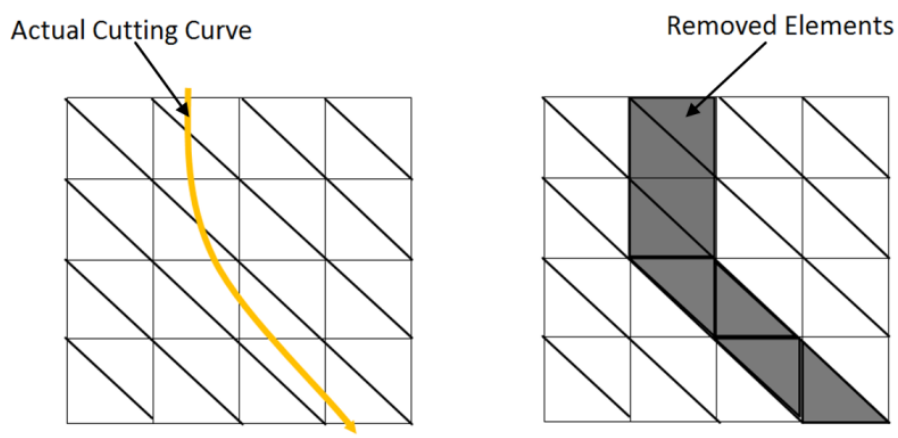

Figure 1.11 Sample cut with element removal algorithm.

\section{Delaunay cutting (DC)}

Delaunay Triangulation (DT) is a popular criterion for generating a well-shaped triangulation of a given set of points and is applied in DC [72] to reshape the mesh elements along the sampled cutting path. In DT an illegal edge belongs to a triangle whose circumcircle contains the opposite vertex of a neighboring triangle (Figure 1.12-a). By changing the edge to connect the other diagonal (an operation called 'flipping'), the new edge will satisfy the DT criterion (Figure 1.12-b).
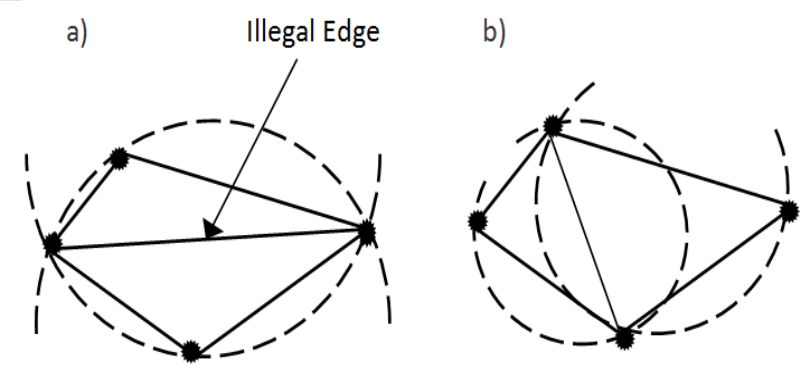

Figure 1.12 Flipping operation in DT [72]. a) Illegal edge in DT. b) After flipping. 
Figure 1.13-b shows an example of flipping when applying Delaunay cutting in Figure 1.13-a. This method keeps the mesh density low and element quality high, but can change the surface shape when performing flipping in 3D [72]. DC delays the displaying of the cut, and this affects real-time display of the cutting path. In particular, at the beginning of the cutting operation, users lose extremely important visual feedback indicating when the blade has punctured the eardrum [27].
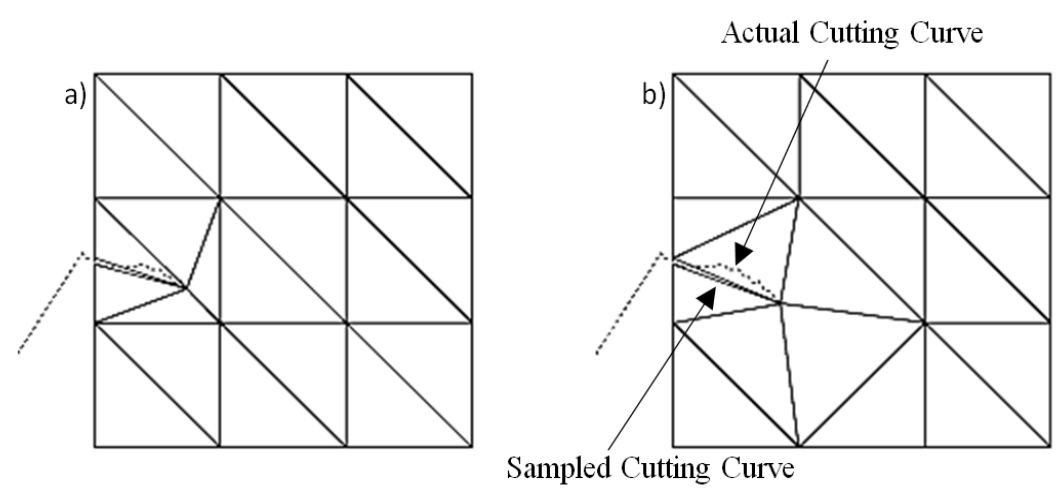

Figure 1.13 A demonstration of DC [72]. a) The start of cutting using DC. b) After flipping in $D C$.

\section{Backward longest-size refinement based cutting (BLSRBC)}

The BLSRBC method uses the Backward Longest-Side Refinement Algorithm (BLSRA) to split triangles around the reference node on an existing cutting path [73, 74]. As an illustration, Figure 1.14-a shows a mesh and an actual cutting curve; the cutting curve is acquired by the input device (e.g., haptic arm). Figure 1.14 illustrates one step of BLSRBC with only the reference node shown being considered. The edges of all triangles that share the reference node are split if their lengths are above a user-specified range. For example, the edge shared by triangles $\mathrm{t} 2$ and $\mathrm{t} 3$ is split as shown in (b). The 
splitting of triangles continues as shown in Figure 1.14-c, d. After this, node 3 in Figure 1.14- $\mathrm{d}$ is considered as the next reference node.

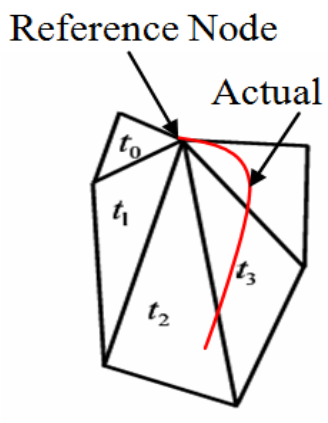

a)

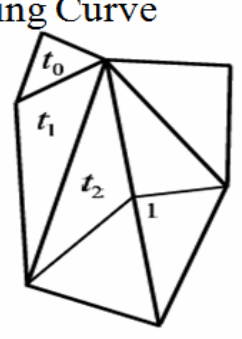

b)

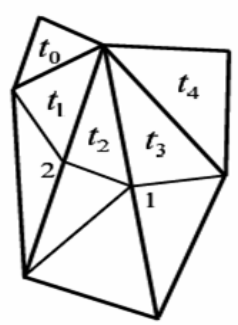

c)

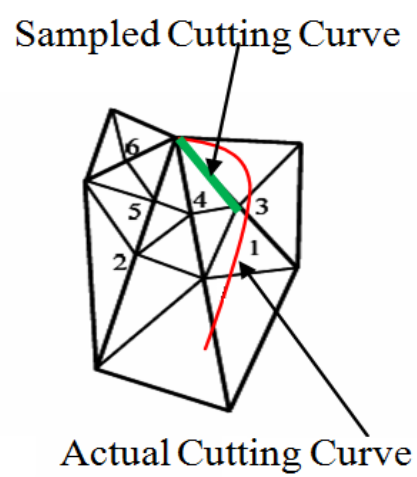

d)

Figure 1.14 A demonstration of BLSRA [74]. The red curve is not displayed in the simulation but is shown here for reference. a) The original mesh with actual cutting curve. b) - c) Splitting of triangles. d) Refined triangles and sampled cutting path.

BLSRBC does not change the shape of the surface being cut. The density of the triangles can increase dramatically along the cutting path which can slow down performance, but the spatial extent around which triangle density is increased can be controlled by a parameter. Simulated cuts made using this algorithm can be smooth because of the increase in the number of triangles. However, a zig-zag sampled cutting curve will emerge inevitably when the next selected node is not close enough to the actual cutting curve. Although this can be remedied by drastically increasing triangle density, this solution can slow down performance to the point where the algorithm is no longer interactive.

\section{Subdivision cutting (SC)}

The basic SC approach splits the triangles along the sampled cutting curve [75, 76], rather than using a refinement strategy like DC and BLSRBC. The sampled cutting curve 
is formed by line segments whose pairs of endpoints are the approximate intersections of the curve and the edges of mesh triangles. For example, in Figure 1.15, ABCEDFG is the sampled cutting curve. The triangle LMN is split by segment $\mathrm{AB}$ and re-meshed by shaded triangles 1,2 , and 3 . The algorithm attempts to keep the sampled cutting curve smooth.

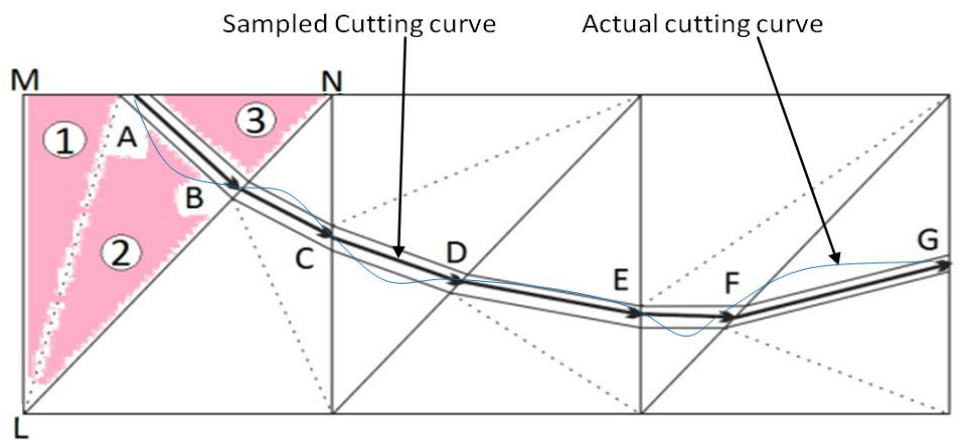

Figure 1.15 A demonstration of SC. The dotted lines are new edges for the re-meshed triangles.

However, if the distance between a pair of vertices, e.g., $\mathrm{E}$ and F, becomes small and is below the limit of the spatial resolution of the haptic arm, the segment EF will be missed, resulting in part of the cutting path being lost. A series of segments can be lost in a fast cutting operation. In [75], the lost segments are made up by recursively calling the basic SC algorithm to split the missed triangles.

\subsubsection{Hardware and software}

In this section, the hardware and software used in existing simulators in Otolaryngology are described as a starting point for the development of the proposed myringotomy simulator. 


\section{Hardware}

VR-based simulators are supported by high performance graphic workstations or a personal computer (PC). With regards to simulators for Otolaryngology, ES3 [77] and the Ohio University simulator [41] use Silicon Graphics workstations. Voxel-Man [78] and the Stanford simulator [79] use high performance PCs. CSIRO (virtual reality ear surgery training system) has a networked structure to support interactive processes among different users in the same simulation [80].

Haptic arms are usually used for tracking the movement of the surgical instruments and rendering force feedback originating from cutting, drilling, and other surgical operations. Ohio [41], Voxel-Man [78] and Stanford [79], simulators are equipped with two PHANTOM Omni haptic arms (Geomagic, Inc., Wilmington, MA). For rendering the surgical view, binocular views of the synthesized surgical field are used in the Ohio University simulator, whereas shuttered glasses are used by Voxel-Man to render 3D graphics.

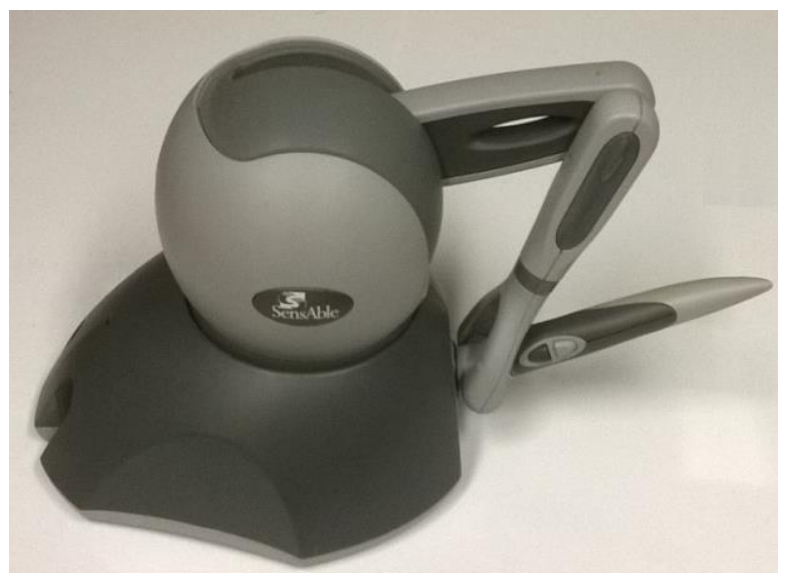

Figure 1.16 Omni haptic arm. 
As mentioned in section 1.5.2, at Western University an Omni haptic arm (see Figure 1.16) was used in myringotomy simulations for $3 \mathrm{D}$ positioning and to provide force feedback. The relevant specifications are documented in Table 1.1. The positioning accuracy and resolution and the size of the workspace are well suited for myringotomy simulation. The contact forces involved when contacting the eardrum with a myringotomy blade are miniscule, and cannot be rendered using this haptic arm or any other existing device. However, surgeons do not perceive any force feedback from the eardrum when cutting. Other sources of tactile feedback in the procedure occur when touching the ear canal wall and when inserting the tube into the incision; these can be simulated using the Omni.

Table 1.1 Specifications of the PHANTOM Omni haptic arm [81].

\begin{tabular}{|l|l|}
\hline Force Feedback Workspace & $>160 \mathrm{~W} \times 120 \mathrm{H} \times 70 \mathrm{~d} \mathrm{~mm}$ \\
\hline Nominal Position Resolution & $\sim 0.055 \mathrm{~mm}$ \\
\hline Backdrive Friction & $<0.26 \mathrm{~N}$ \\
\hline Maximum Exertable Force & $3.3 \mathrm{~N}$ \\
\hline Continuous Exertable Force & $>0.88 \mathrm{~N}$ \\
\hline Force Feed Back & $\mathrm{X}, \mathrm{Y}, \mathrm{Z}$ \\
\hline Stiffness & X axis $>1.26 \mathrm{~N} / \mathrm{mm}$ \\
& Y axis $>2.31 \mathrm{~N} / \mathrm{mm}$ \\
& Z axis $>1.02 \mathrm{~N} / \mathrm{mm}$ \\
\hline
\end{tabular}

\section{Software platforms}

CHAI3D [82] is an open source C++ platform developed by Stanford University and is used in their simulators. It supports algorithms for surgical simulation, e.g., deformation modeling and force feedback. The CSIRO simulator is built on the Reachin API [83]. The Reachin API provides an extensive library of pre-written code to support haptics-enabled applications. SOFA [84] is another open source framework primarily targeted at real-time simulation, with an emphasis on medical simulation. It is mostly intended for the research 
community to help develop newer algorithms, but can also be used as an efficient prototyping tool.

Game engines have garnered significant attention for medical simulation because of their excellent support for high-quality real-time rendering of interactive virtual environments [85]. In addition to rendering, it is now possible to integrate a high quality real-time haptics system into a digital gaming engine, e.g. haptic interfaces have been integrated into the Unity3D gaming engine (Unity Technologies, San Francisco, CA) [86, 87]. Even though complicated physics-based models for the simulation of tissue deformation and cutting have not been directly implemented in gaming engines, the basic interaction with soft tissues can be simulated by using mass spring models [85], which are available in many gaming engines. Also most game engines have capabilities for collision detection.

Moreover, commercial game engines are cost effective, well documented and have a large community of users who can provide support. The availability of these features indicates that gaming engines may soon be used to create serious surgical simulators.

\subsubsection{Validation methods}

Scientific validation is a common concern in area such as psychology. "The problem of validity is that of whether a test really measures what it purports to measure" [88]. It relates to the property of "being true, correct, and in conformity with reality." Extending to the field of surgical simulation, it is widely used in evaluating simulators before they can be integrated into training programs effectively. 


\section{Face and content validity study}

As summarized by Shout et al. [89], the methods of validity studies can be categorized into two kinds - subjective and objective approaches. Face and content validity belong to subjective approach of validity, which examine the opinions of the selected participants (subjects) with regard to the simulator. Face validity refers to the degree to which a simulation appears like the real situation [90]. Content validity indicates the level to which a simulator covers all aspects of the subject matter being taught $[89,91]$. Participants in face and content validity studies can be both experts (surgeons) and juniors (residents) since subjective impressions of both groups are important. The test can be achieved by permitting two groups of participants (expert surgeons and junior residents) to perform a procedure using the simulator and then asking them to complete a questionnaire about their experience with the simulator. See e.g., Bajka et al. [92].

In Otolaryngology, nine face and content validity studies were reported for four simulators - Voxel-Man, Mediseus, ES3 and Western University's myringotomy simulator [29]. Seven out of nine studies used questionnaires with Likert scale responses as the measurement method. The remaining two studies used objective assessment tools, for example, OSATS (Objective Structured Assessment of Technical Skill), and metrics to reflect users' evaluation. In all the studies, the number of the participants recruited was less than 20 except in one study which had 85 participants [29]. In the past three face validity studies for Western's myringotomy simulator, the participants recruited were fewer than twelve in each study. The simulator was evaluated by using questionnaires, and each question was answered using a 7-point Likert scale ranging from "strongly 
disagree" to "strongly agree". Descriptive statistics were used to summarize the data and identify strengths, weaknesses and future directions.

\section{Construct validity study}

Construct validity is an objective assessment approach which generally involves experiments for "evaluating a testing instrument based on the degree to which test items identify the quality, ability, or trait it was designed to measure" [91]. This form of validity consists of convergent and discriminant validity. Convergent validity is defined as the degree to which measures that are supposedly related agree with each other. On the other hand, discriminant validity refers to the degree of agreement between measures that should not be related [93]. In discriminant validity study of a surgical simulator, the experiments are designed to ascertain whether a simulator can differentiate different levels of expertise. Usually, participants are grouped depending on their experience. Each participant is asked to perform a surgery using the simulator being validated and repeat the simulated surgery a certain number of times. Often kinematic operation data of users are collected by the simulator. Then metrics are applied to quantitatively measure the user's performance based on the collected data. Statistical analysis is applied to evaluate the level of the variance between groups.

In the area of Otolaryngology, automatic metrics have been designed for simulators such as the Stanford Surgical Simulator [39], the Voxel-Man Tempsurg [94] and the Mesiseus Surgical Drilling Simulator [95] for temporal bone surgery, and MSESS [96] and ES3 [97] for sinus surgery. The time to completion was reported in [94-97] as an important metric for measuring users' proficiency. Efficiency measurements such as the magnitude of the drilling force $[39,94-96]$, the length of the motion trajectory [96], the drilling velocity 
[39], the amount of tissue removed $[39,94,96]$ and the amount (or frequency) of the operations [96] such as number of washes and number of drill activations were calculated. The level of injury or dangerous operations was also evaluated using metrics [39, 94-96]. For each metric, mean scores with $95 \%$ confidence intervals were determined for user trials to see if training can improve the skills of the participants [96, 97]. Dynamic differences between expert and novice groups were compared, e.g., using analysis of variance [95-97] and the Mann-Whitney U test [96] to see if the simulator can distinguish the different levels of experience.

\section{VR-to-OR transference}

The study of skill transfer from the VR training environment to the operating room (OR) is the most rigorous form of validation, and involves investigating if the skills acquired from VR training environment can positively transfer to the OR [98]. Such a study can be conducted by comparing the outcomes (e.g., measures of surgical task performance or morbidity and discomfort of the patients [98]) between two groups of trainees (e.g., a group trained using the traditional approach and another group whose training is augmented by simulation [98]). The results are used to establish if surgical practice can benefit from VR-based training without being negatively affected by the simulated virtual environment. A VR-to-OR transference study is usually the final stage of validating the training capabilities of a simulator.

Based on attempts to establish skills transference, claims have been made that some simulators provide positive impact on resident performance in the OR, e.g., ES3 in sinus surgery [99], and CSIRO [100] and Voxel Man [101] for the temporal bone. However, a critical examination of the results [98] indicates that in these studies the VR-to-OR skill 
transference was not fully validated because of challenges in conducting randomised controlled trials and the limited number of participants involved.

\subsection{Objectives}

The long-term objective of the ABL is to develop and validate a full myringotomy simulator and deploy it in surgical residency programs in Otolaryngology departments. As noted in Section 1.4, some aspects of this long-term objective have been reached. The specific focus of this proposed doctoral work is to simulate myringotomy with ventilation tube placement and to validate some aspects of the simulator. The specific objectives are:

I. Design and implement a VR-based simulator for training residents in myringotomy and ventilation tube placement. A major focus of this effort is the implementation of the system using existing simulation software libraries that are readily available, well supported and extensible.

II. Perform face and content validity studies for the simulator. The aim is to determine whether the simulator's appearance and the training potential are acceptable, especially from the perspectives of junior residents and expert surgeons.

III. Identify and implement metrics to assess trainee performance in myringotomy surgical simulation. Then validate these metrics by performing a construct validity study for the simulator. 


\subsection{Thesis outline and contributions}

As noted, the long-term objective of the ABL is to develop a surgical simulator for myringotomy with tube placement and completely validate the simulator and transfer it to residency programs. This work focuses on further development of the simulator and performing initial validity testing, including face and content validity and discriminant validity. Transference testing is beyond the scope of this work but is required for acceptance into training curricula.

The contributions of this work are three-fold: (1) the development of a simulator on a common digital gaming platform that goes beyond previous simulator implementations by including microscope manipulation, speculum positioning, tube placement and computation of performance metrics; (2) face and content validity testing; and (3) identification, implementation and evaluation of quantitative performance metrics. 


\subsection{References}

1 W. Kaplan, V. J. Wirtz, A. Mantel-Teeuwisse, P. Stolk, B. Duthey, R. Laing, "Priority Diseases and Reasons for Inclusion," 2013. [Accessed: July 30, 2015]. [Online]. Available: http://www.who.int/medicines/areas/priority_medicines /MasterDocJune28_FINAL_Web.pdf?ua=1.

2 E. M. Thomas, "Recent trends in respiratory infections, ear infections and asthma among young Canadian children," Component of Statistics Canada Catalogue no. 82-003-XPE.Health Reports, vol. 21, no. 4, 2010.

3 G. Petit, P. D. Wals, B. Law, T. Tam, L. J. Erickson, M. Guay, A. Framarin, "Epidemiological and economic burden of pneumococcal diseases in Canadian children," The Canadian Journal of Infectious Diseases \& Medical Microbiology, vol. 14, no. 4, pp. 215-220, 2003.

4 R. Pokras, L. J. Kozak, E. McCarthy. Vital and Health Statistics, vol. 13, no. 132, 1997.

5 S. Baer, H. Williams, A. McCOMBE, "A model for instruction in myringotomy and grommet insertion," Clinical Otolaryngology Allied Sciences, vol. 15, no. 4, pp. 383384, 1990.

6 File:HumanEar.jpg, The human ear, Wikipedia Commons, [Accessed: July 30, 2014]. [Online]. Available: http://commons.wikimedia.org/wiki/File:HumanEar.jpg.

7 "Auricle (anatomy)", Wikipedia, [Accessed: July 30, 2015]. [Website]. Available: https://en.wikipedia.org/wiki/ Auricle_(anatomy).

8 W. J. Staab, "Ear Canal Dimensions", 2014. [Accessed: July 30, 2015]. [Website]. Available: http://hearinghealthmatters.org/waynesworld/2014/ear-canal-dimensions/.

9 W. J. Staab, "The Human Ear Canal - V", 2014. [Accessed: July 30, 2015]. [Website]. Available: http://hearinghealthmatters.org/waynesworld/2014/human-earcanal-v/.

10 W. J. Staab, "The Human Ear Canal - III", 2014. [Accessed: July 30, 2015].

[Website]. Available: http://hearinghealthmatters.org/waynesworld/2014/human-earcanal-iii/.

11 P. W. Alberti, "The anatomy and physiology of the ear and hearing", 1918.

[Accessed: July 30, 2015]. [Online]. Available:

http://www.who.int/occupational_health/publications/noise2.pdf,

12 F. Salvinelli, M. Maurizi, S. Calamita, L. D'Alatri, A. Capelliet, A. Carbone, "The external ear and the tympanic membrane. A three-dimensional study", Scandinavian Audiology, vol. 20, no. 14, pp. 253-256, 1991.

13 S. Van der Jeught, J. J. Dirckx, J. R. Aerts, A. Bradu, A.G Podoleanu, J. A. Buytaert, "Full-field thickness distribution of human tympanic membrane obtained with optical coherence tomography", Journal of the Association for Research in Otolaryngology, vol. 14, no. 4, pp. 483-494, 2013.

14 File:Gray909.png, The human eardrum, Wikipedia, [Accessed: July 30, 2014]. [Website]. Available: http://en.wikipedia.org/wiki/File:Gray909.png.

15 H. Gray, "Anatomy of the Human Body", 1918. [Book Chapter online: 1d. 2. The Middle Ear or Tympanic Cavity]. [Accessed: July 30, 2015]. [Website]. Available: http://www.bartleby. com/107/230.html. 
16 Paste17.jpg, The illustration of myringotomy, kveinhomd.com, [Accessed: July 30, 2014]. [Online]. Available: http://www.kevinhomd.com/images/paste17.jpg.

17 J. K. Fuller. Surgical Technology: Principles and Practice (6th edition), pp. 690. St. Louis, Missouri: Elsevier/Saunders, 2013.

18 M. L. Montague, M. S. W. Lee, S. S. M. Hussain, "Human error identification: an analysis of myringotomy and ventilation tube insertion," Archives of Otolaryngology-Head and Neck Surgery, vol. 130, no. 10, pp. 1153-1157, 2004.

19 T. E. Norris, S. W. Cullison, S. D. Fihn, "Teaching procedural skills," Journal of General Internal Medicine, vol. 12, pp. 64-70, 1997.

20 T. Walker, S. Duvvi, and B. N. Kumar, "The Wigan Grommet Trainer," Clinical Otolaryngology, vol. 31, no. 4, pp. 349-350, 2006.

21 M. Duijvestein, and J. Borgstein, "The Bradford Grommet Trainer," Clinical Otolaryngology, vol. 31, no. 2, pp. 163-163, 2006.

22 A. Leong, S. Kundu, P. Martinez-Devesa, C. Aldren, "Artificial ear: a training tool for grommet insertion and manual dexterity," ORL: Journal for Oto - Rhino Laryngology and Its Related Specialties, vol. 68, no. 2, pp. 115-117, 2006.

23 P. Hong, A. N. Webb, G. Corsten, J. Balderston, R. Haworth, K. Ritchie, E. Massoud. "An anatomically sound surgical simulation model for myringotomy and tympanostomy tube insertion," International Journal of Pediatric Otorhinolaryngology, vol. 78, pp. 522-529, 2008.

24 P. G. Volsky, B. B. Hughley, S. M. Peirce, B. W. Kesser, "Construct validity of a simulator for myringotomy with ventilation tube insertion, " Otolaryngology-Head and Neck Surgery, vol. 141, pp. 603-608, 2009.

25 B. Wheeler, P. C. Doyle, S. Chandarana, S. Agrawal, M. Husein, H. M. Ladak, "Interactive computer-based simulator for training in blade navigation and targeting in myringotomy," Computer Methods Programs in Biomedicine, vol. 98, no. 2, pp. 130-139, 2010.

26 L. J. Sowerby, G. Rehal, M. Husein, P. C. Doyle, S. Agrawal, H. M. Ladak, "Development and face validity testing of a three-dimensional myringotomy simulator with haptic feedback," Journal of Otolaryngology: Head and Neck Surgery, vol. 39, no. 2, pp. 122-129, 2010.

27 A. K. Ho, H. Alsaffar, P. C. Doyle, H. M. Ladak, S. K. Agrawal, "Virtual reality myringotomy simulation with real-time deformation: development and validity testing," The Laryngoscope, vol. 122, no. 8, pp. 1844-1851, 2012.

28 M. P. Fried, J. I. Uribe, b. Sadoughi, "The role of virtual reality in surgical training in otorhinolaryngology," Current Opinion in Otolaryngology \& Head and Neck Surgery, vol. 15, no. 3, pp. 163-169, 2007.

29 A. Arora, L.Y. Lau, Z. Awad, A. Darzi, A. Singh, N. Tolley, "Virtual reality simulation training in Otolaryngology," International Journal of Surgery, vol. 12, no. 2, pp. 87-94, 2014.

30 S. Weghorst, C. Airola, P. Oppenheimer, C. V. Edmond, T. Patience, D. Heskamp, J. Miller, "Validation of the Madigan ESS Simulator," Studies in Health Technology and Informatics, vol. 50, pp. 399-405, 1998.

31 S. M. Anil, Y. Kato, M. Hayakawa, K. Yoshida, S. Nagahisha, T. Kanno, "Virtual 3dimensional preoperative planning with the Dextroscope for excision of a 4 th 
ventricular ependymoma," Minimally Invasive Neurosurgery, vol. 50, pp. 65-70, 2007.

32 M. Audette, H. Delingette, A. Fuchs, O. Astley, K. Chinzei, "A topologically faithful, tissue-guided, spatially varying meshing strategy for computing patient-specific head models for endoscopic pituitary surgery simulation," Studies in Health Technology and Informatics, vol. 119, pp. 22-27, 2006.

33 B. Tolsdorff, A. Pommert, K. H. Höhne, A. Petersik, B. Pflesser, U. Tiede, R. Leuwer, "Virtual reality: a new paranasal sinus surgery simulator," Laryngoscope, vol. 120, pp. 420-426, 2010.

34 R. Varshney, S. Frenkiel, L. H. Nguyen, M. Young, D. R. Maestro, A. Zeitouni, M. A. Tewfik, "Development of the McGill simulator for endoscopic sinus surgery: a new high-fidelity virtual reality simulator for endoscopic sinus surgery," The American Journal of Rhinology \& Allergy, vol. 28, no. 4, pp. 330-334, 2014.

35 S. Delorme, D. Laroche, R. DiRaddo, R. Del Maestro, "NeuroTouch: a physicsbased virtual simulator for cranial microneurosurgery training," Neurosurgery, vol. 71, pp. 32-42, 2012.

36 G. Rosseau, J. Bailes, R. Del Maestro, A. Cabral , N. Choudhury, O. Comas, P. Debergue, G. De Luca, J. Hovdebo, D. Jiang, D. Laroche, A. Neubauer, V. Pazos, F. Thibault, R. Diraddo, "The development of a virtual simulator for training neurosurgeons to perform and perfect endoscopic endonasal transsphenoidal surgery," Neurosurgery, vol. 73(Suppl 1), pp. 85-93, 2013.

37 R. Varshney, S. Frenkiel, L. H. P. Nguyen, M. Young, R. Del Maestro, A. Zeitouni, E. Saad, W. R. J. Funnell, M. A. Tewfik, National Research Council Canada, "The McGill simulator for endoscopic sinus surgery (MSESS): a validation study," Journal of Otolaryngology-Head and Neck Surgery, vol. 43, pp. 40, 2014.

38 D. Morris, C. Sewell, F. Barbagli, K. Salisbury, N. H. Blevins, S. Girod, "Visuohaptic simulation of bone surgery for training and evaluation," IEEE Computer Graphics and Applications, vol. 26, pp. 48-57, 2006.

39 C. Sewell, D. Morris, N. H. Blevins, S. Dutta, S. Agrawal, F. Barbagli, K. Salisbury, "Providing metrics and performance feedback in a surgical simulator," Computer Aided Surgery, vol. 13, pp. 63-81, 2008.

40 G. J. Wiet, D. Stredney, D. Sessanna, J. A. Bryan, D. B. Welling, P. Schmalbrock, "Virtual temporal bone dissection: an interactive surgical simulator," Otolaryngology-Head and Neck Surgery, vol. 127, pp. 79-83, 2002.

41 G. J. Wiet, D. Stredney, T. Kerwin, B. Hittle, S. A. Fernandez, M. Abdel-Rasoul, D. B. Welling, "Virtual temporal bone dissection system: development and testing," The Laryngoscope, vol. 122(Suppl. 1), pp. S1-S12, 2012.

42 VOXEL-MAN TempoSurg, The Voxel-Man Group, [Accessed: July 30, 2014]. [Website]. Available: http://www.voxel-man.com/simulator/tempo/.

43 A. Arora, S. Khemani, N. Tolley, A. Singh, J. Budge, D. A. Varela, H. W. Francis, A. Darzi, N. I. Bhatti, "Face and content validation of a virtual reality temporal bone simulator," Otolaryngology-Head and Neck Surgery, vol. 146, pp. 497-503, 2012.

44 "OGRE 3D: Open source graphics engine," [Accessed: July 30, 2014]. [Website]. Available: http://www.ogre3d.org. 
45 "ODE: Open Dynamics Engine," [Accessed: July 30, 2014]. [Website]. Available: http:// www.ode.org.

46 "OpenHaptics Toolkit," [Accessed: January 30, 2014]. [Website]. Available: http://www.sensable.com/products-openhaptics-toolkit.htm.

47 "CHAI3D," [Accessed: January 30, 2014]. [Website]. Available: http://www.chai3d.org/.

48 M. Vasilescu, D. Terzopoulos, "Adaptive meshes and shells: irregular triangulation, discontinuities, and hierarchical subdivision," Proceedings of the IEEE Computer Society Conference on Computer Vision and Pattern Recognition, pp. 829-832, 1992.

49 S. F. Frisken-Gibson, "Using linked volumes to model object collisions, deformation, cutting, carving, and joining," Visualization and Computer Graphics, IEEE Transactions on, vol. 5, no. 4, pp. 333-348, 1999.

50 J. Brown, S. Sorkin, J. Latombe, K. Montgomery, M. Stephanides, "Algorithmic tools for real-time microsurgery simulation," Medical Image Analysis, vol. 6, no. 3, pp. 289-300, 2002.

51 B. Qiao, G. Chen, X. Ye, "The Research of soft tissue deformation based on massspring model," International Conference on Mechatronics and Automation, pp. 4655-4660, 2009.

52 S. Zhang, L. Gu, W. Liang, P. Huang, J. Boehm, and J. Xu, "The framework for realtime simulation of deformable soft-tissue using a hybrid elastic model," Biomedical Simulation. vol. 4072, pp. 75-83, 2006.

53 J. Zhang, L. Gu, P. Huang, J. Dworzak, F. Chen, and X. Kong, "Real-time cutting and suture simulation using hybrid elastic model," 29th Annual International Conference of the IEEE on Engineering in Medicine and Biology Society, pp. 36463649, 2007.

54 F. Chen, L. Gu, P. Huang, J. Zhang, and J. Xu, "Soft tissue modeling using nonlinear mass spring and simplified medial representation," 29th Annual International Conference of the IEEE on Engineering in Medicine and Biology Society, pp. 5083-5086, 2007.

$55 \mathrm{~S}$. Xu, X. Liu, and H. Zhang, "Simulation of soft tissue using mass-spring model with simulated annealing optimization," IEEE International Conference on Automation and Logistics, pp. 1543-1547, 2009.

56 L. Zhu, X. Ye, X. Ji'er, Y. Gu, and S. Guo, "A Real-time deformation modeling scheme of soft tissue for virtual surgical," IEEE International Conference on Information and Automation (ICIA), pp. 771-775, 2010.

57 Y. Yang, R. Xiao, and Z. He, "Real-time deformations simulation of soft tissue by combining mass-spring model with pressure based method," 3rd International Conference on Advanced Computer Control (ICACC), pp. 506-510, 2011.

58 C. Basdogan, M. Sedef, M. Harders, and S. Wesarg, "VR-based simulators for training in minimally invasive surgery," Computer Graphics and Applications, IEEE, vol. 27, no. 2, pp. 54-66, 2007.

59 S. Cotin, and H. Delingette, "Real-time surgery simulation with haptic feedback using finite elements," Proceedings of the IEEE International Conference on Robotics and Automation, vol. 4, pp. 3739-3744, 1998. 
60 G. Picinbono, H. Delingette, and N. Ayache, "Nonlinear and anisotropic elastic soft tissue models for medical simulation," Proceedings of the IEEE International Conference on Robotics and Automation, vol. 2, pp. 1370-1375, 2001.

61 X. Wu, M. S. Downes, T. Goktekin, and F. Tendick, "adaptive nonlinear finite elements for deformable body simulation using dynamic progressive meshes," Computer Graphics Forum, vol. 20, no. 3, pp. 349-358, 2001.

62 I. Khalaji, K. Rahemifar, A. Samani, "Statistical finite element analysis", Proceedings of 30th Annual International IEEE Conference on Engineering in Medicine and Biology Society, pp. 5577-5580, 2008.

63 H. Delingette, S. Cotin, and N. Ayache, "A hybrid elastic model allowing real-time cutting, deformations and force-feedback for surgery training and simulation," Proceedings on Computer Animation, pp. 70-81, 1999.

64 S. Cotin, H. Delingette, and N. Ayache, "Real-time elastic deformations of soft tissues for surgery simulation," Visualization and Computer Graphics, IEEE Transactions on, vol. 5, no. 1, pp. 62-73, 1999.

65 S. De, Y. Lim. "Physically realistic virtual surgery using the point-associated finite field (paff) approach," Presence: Teleoperators and Virtual Environments, vol. 15, no. 3, pp. 294-368, 2006.

66 I. F. Costa, and R. Balaniuk, "LEM - an approach for real time physically based soft tissue simulation," Proceedings of the IEEE International Conference on Robotics and Automation, vol. 3, pp. 2337-2343 vol.3, 2001.

67 K. Sundaraj, and C. Laugier, "Physically realistic simulation of large deformations using lem for interactive applications," IEEE/RSJ International Conference on Intelligent Robots and Systems, vol. 3, pp. 3054-3059, 2002.

68 R. Balaniuk, and K. Salisbury, "Dynamic simulation of deformable objects using the long elements method," Proceedings of the 10th Symposium on Haptic Interfaces for Virtual Environment and Teleoperator Systems, pp. 58-65, 2002.

69 Y. Li, and K. Brodlie, "Soft object modelling with generalised chainmail -extending the boundaries of web-based graphics," Computer Graphics Forum, vol. 22, no. 4, pp. 717-727, 2003.

70 S. Nefti, and E. M. Abulgasem, "Neural networks approach for soft tissue modelling," International Conference on Emerging Technologies, pp. 200-205, 2009.

71 A. Radetzky, A. Nurnberger, M. Teistler, and D. P. Pretschner, "Elastodynamic shape modeling in virtual medicine," Proceedings of the International Conference on Shape Modeling and Applications, pp. 172-178, 276, 1999.

72 H. W. Nienhuys, and A. F. van der Stappen, "A delaunay approach to interactive cutting in triangulated surfaces," Springer Tracts Adv Robot, vol. 7, pp. 113-130, 2004.

73 H. T. Tanaka, Y. Tsujino, T. Kamada, and Huynh Quang Huy Viet, "Bisection refinement-based real-time adaptive mesh model for deformation and cutting of soft objects," 9th International Conference on Control, Automation, Robotics and Vision, pp. 1-8, 2006.

74 H. Q. Huy Viet, T. Kamada, and H. T. Tanaka, "An algorithm for cutting 3d surface meshes," 18th International Conference on Pattern Recognition, vol. 4, pp. 762-765, 2006. 
75 C. D. Bruyns, and S. Senger, "Interactive cutting of 3D surface meshes," Computers Graphics, vol. 25, no. 4, pp. 635-642, 2001.

76 Y. Zhou, D. Zheng, and D. Hu, "Research of virtual cutting technology based on haptic feedback," IEEE 10th International Conference on Computer-Aided Industrial Design Conceptual Design, pp. 2197-2201, 2009.

77 "ES3," [Accessed: January 30, 2014]. [Website]. Available:

http://www.virtualsurgery.vision.ee.ethz.ch//LockheedMartin/MadiganEndoscopicSi nusSurgery

78 "VoxeL-Man", The Voxel-Man Group, [Accessed: January 30, 2014]. [Website]. Available: http://www.voxel-man.com/simulator/tempo/.

79 "Stanford simulator," [Accessed: January 30, 2014]. [Website]. Available: http://techhouse.brown.edu/ dmorris/projects/bonesim/.

80 S. J. O'Leary, M. A. Hutchins, D. R. Stevenson, C. Gunn, A. Krumpholz, G. Kennedy, M. Tykocinski, M. Dahm, B. Pyman, "Validation of a networked virtual reality simulation of temporal bone surgery," The Laryngoscope, vol. 118, no. 6, pp. 1040-1046, 2008.

81 "Sensable Phantom Omni Specifications - Geomagic," [Accessed: March 22, 2014]. [Website]. Available: http://www.geomagic.com/archives/phantomomni/specifications/.

82 "CHAI3D," [Accessed: May 30, 2013]. [Website]. Available: http://www.chai3d.org/.

83 "Reachin API," [Accessed: January 30, 2013]. [Website]. Available: http://www.reachin.se/products/reachinapi/.

84 "SOFA," [Accessed: January 30, 2013]. [Website]. Available: http://www.sofaframework.org/home.

85 S. Marks, J. Windsor, and B. Wünsche, "Evaluation of game engines for simulated surgical training," Proceedings of the 5th international conference on Computer graphics and interactive techniques in Australia and Southeast Asia, pp. 273-280, 2007.

86 "PRWeb: CyberTouch haptic glove used to show feasibility of 3D maintenance training and display system," [Accessed: January 30, 2014]. [Website]. Available: http://www.prweb.com /releases /2012/11/ prweb10178291.htm.

87 "RNI: RNI demonstrates HitBox haptics systems. Research network incorporated," [Accessed: January 30, 2014]. [Website]. Available: http://www.resrchnet.com/category/rni-news/.

88 T. L. Kelley, Interpretation of educational measurements. New York: Macmillan. pp. 14, 1927.

89 B. M. A. Schout, A. J. M. Hendrikx, F. Scheele, B. L. H. Bemelmans, A. J. J. A. Scherpbier, "Validation and implementation of surgical simulators: a critical review of present, past, and future," Surgical endoscopy, vol. 24, no. 3, pp. 536-546, 2010.

90 F. J. Carter, M. P. Schijven, R. Aggarwal, T. Grantcharov, N. K. Francis, G. B. Hanna, J. J. Jakimowicz, "Consensus guidelines for validation of virtual reality surgical simulators," Surgical Endoscopy, vol. 19, no. 12, pp. 1523-1532, 2005. 
91 A. G. Gallagher, E. M. Ritter, R. M. Satava, "Fundamental principles of validation, and reliability rigorous science for the assessment of surgical education and training," Surgical Endoscopy, vol. 17, no. 10, pp. 1525-1529, 2003.

92 M Bajka, S Tuchschmid, M.Streich, D. Fink, G. Székely, M. Harders, "Evaluation of a new virtual-reality training simulator for hysteroscopy," Surgical endoscopy, vol. 23, no. 9, pp. 2026-2033, 2009.

93 R. Chin, B. Y. Lee. Principles and Practice of Clinical Trial Medicine, pp. 78. St. Louis, Missouri : Elsevier, 2008.

94 S. Khemani, A. Arora, A, Singh, N, Tolley, A. Darzi, "Objective skills assessment and construct validation of a virtual reality temporal bone simulator," Otology \& Neurotology, vol. 37, no.7, pp. 1225-1231, 2012.

95 Y. C. Zhao, G. Kennedy, R. Hall, S. O'Leary, "Differentiating levels of surgical experience on a virtual reality temporal bone simulator," Otolaryngology-Head and Neck Surgery, vol. 143, no. 5 (Supplement), pp. S30-S35, 2010.

96 R. Varshney, S. Frenkiel, L. H. P. Nguyen, M. Young, R. Del Maestro, A. Zeitouni, E. Saad, W. R. J. Funnell, M. A. Tewfik, National Research Council Canada, "The McGill simulator for endoscopic sinus surgery (MSESS): a validation study, " Journal of Otolaryngology-Head and Neck Surgery, vol. 43, pp. 40, 2014.

97 M. P Fried, B. Sadoughi, S.J. Weghorst, M. Zeltsan, H. Cuellar, J. I. Uribe, C. T. Sasaki, D. A. Ross, J. B. Jacobs, R. A. Lebowitz, R. M. Satava, "Construct validity of the endoscopic sinus surgery simulator simulator II. Assessment of discriminant validity and expert benchmarking," Archives of Otolaryngology - Head \& Neck Surgery, vol. 133, no.4, pp. 350-357, 2007.

98 Australian Safety \& Efficacy Register of New Interventional Procedures - Surgical (ASERNIP-S), "The Systematic Review of Surgical Simulation for Training: Skills Transfer to the Operating Room," ASERNIP-S Report No. 61, 2007. [Accessed: September 28, 2015]. [Online]. Available: www.surgeons.org/media/300327/Surgicalsimulation_systematicreview.pdf.

99 M. P Fried, B. Sadoughi, M. J. Gibber, J. B. Jacobs, R. A. Lebowitz, D. A. Ross, J. P. Bent, S. R. Parikh, C. T. Sasaki, S. D.Schaefer, "From virtual reality to the operating room: the endoscopic sinus surgery simulator experiment," Otolaryngology-Head and Neck Surgery, no. 142, pp. 202-207, 2010.

100 Y. C. Zhao, G. Kennedy, K. Yukawa, B. Pyman, S. O’Leary, "Improving temporal bone dissection using self-directed virtual reality simulation: results of a randomized controlled trial," Otolaryngology-Head and Neck Surgery, no. 144, pp. 357, 2011.

101 H. W. Francis, M. U. Malik, D. A. Diaz Voss Varela, M. A. Barffour, W. W. Chien, J. P. Carey , J. K. Niparko, N. I. Bhatti, "Technical skills improve after practice on virtual-reality temporal bone simulator," The Laryngoscope, vol. 122, no. 6, pp. 1385 $-1391,2012$. 


\section{Chapter 2. Virtual-Reality Simulator for Training in Myringotomy with Tube Placement}

This chapter is a post-print of a research paper that was in press at the time of submission

of this thesis: C. Huang, S. K. Agrawal, H. M. Ladak, A virtual-reality simulator for training in myringotomy with tube placement," Journal of Medical and Biological Engineering, in press. The final publication is available at Springer via http://dx.doi.org/10.1007/s40846-016-0124-1.

\subsection{Introduction}

Myringotomy with tube insertion is a very common surgical procedure in which a tiny incision is made in the eardrum and a ventilation tube is inserted to treat middle-ear infections. As this is the first procedure that surgical residents in Otolaryngology learn, there is great potential for damaging the delicate structures of the ear, potentially resulting in poor hearing outcomes [1].

In this surgery, a surgeon uses one hand to hold a speculum and uses the other hand to perform procedures under a surgical microscope such as removing wax, inserting a blade through the narrow ear canal, creating the incision and placing the tube. High levels of dexterity and experience are needed to operate in the small confines of the ear canal.

Surgical simulators based on virtual-reality (VR) technologies have been developed as potential training tools in Otolaryngology, especially for endoscopic sinus surgery [2-5] 
and for temporal bone drilling [6-9]. In contrast to training on an actual patient, VR-based simulators allow surgical residents to practice without any risk to a patient. Moreover, the flexibility of VR systems allows a variety of anatomies and mechanical properties to be simulated, in contrast to several physical simulators that exist for myringotomy [10-13]. VR simulators can also be more readily available and cost-effective in comparison to cadaveric specimens. Finally, unlike physical simulators, VR-simulators can track quantitative performance metrics and provide real-time feedback and tutoring for trainees $[6,8]$.

The Auditory Biophysics Laboratory at Western University has reported on several aspects of VR-based myringotomy simulation. A blade navigation system [14,15] and a system for modeling deformation and cutting of the eardrum [16] were implemented on different software platforms as separate training modules. As the speculum, microscope, and tube insertion were not simulated, trainees were unable to practice the myringotomy procedure. Furthermore, since the simulator was fragmented by the use of various software platforms, integration into one single system was essential to allow an end-toend simulator for surgical residents.

VR-based surgical simulation pursues immersive interactions between users and virtual tissues, providing real-time visual rendering, realistic deformation of the tissues and tactile feedback. Digital gaming engines have excellent support for high quality real-time rendering of interactive virtual environments; therefore, they have garnered significant attention for medical simulation [17]. In addition to rendering, it is now possible to integrate a high quality real-time haptics system into a digital gaming engine [e.g. haptic interfaces have been integrated into the Unity3D gaming engine (Unity Technologies, 
San Francisco, CA)] [18,19]. Even though complicated physics-based models for the simulation of tissue deformation and cutting have not been directly implemented in gaming engines, the basic interaction with soft tissues can be simulated by using massspring models [17], which are available in many gaming engines. The availability of these features indicates that gaming engines may soon be used to create serious surgical simulators.

Myringotomy with tube insertion involves a sequence of discrete steps as described in the literature [1]. In this paper, a new integrated VR-based myringotomy simulator is described that supports surgical tasks such as adjustment of the surgical view by positioning and orienting the speculum and microscope, blade navigation through the speculum and ear canal, cutting of a deformable eardrum model, and inserting a ventilation tube into the incision. The cutting model was redesigned to allow implementation in an existing gaming engine, and new tube insertion algorithms were developed based on the new model for deformation and cutting. The simulator was implemented using the Unity3D gaming engine, and a haptic interface was added to provide force feedback when adjusting the microscope, positioning the speculum, and inserting the tube. Finally, the simulated graphical scenes are displayed in stereoscopic 3D to mimic the actual view through a surgical microscope. This paper is unique in that it integrates both our previous work and the newly developed algorithms into a single unified gaming engine (Unity 3D). 


\subsection{Methods}

\subsubsection{System overview}

The simulator runs on a Z420 HP workstation with a DevinSense Display 300 system (Devinsense Display Solutions, Sundbyberg, Sweden) [20] and the Windows 7 operating system. A Phantom Omni haptic arm (Geomagic, Inc., Morrisville, NC) is employed for 3D positioning of surgical instruments such as the virtual microscope, speculum, blade, forceps, and ventilation tube. The arm also provides force feedback during the various surgical maneuvers to simulate the actual procedure. The Phantom Omni provides 6 degrees of freedom (DOF) in positioning and 3DOF in force feedback. The simulated scenes are displayed on a silver screen mirror. The user can experience an immersive stereoscopic 3D view through a pair of Nvidia active 3D glasses (Nvidia Corp., Santa Clara, CA) as shown in Figure 2.1. 


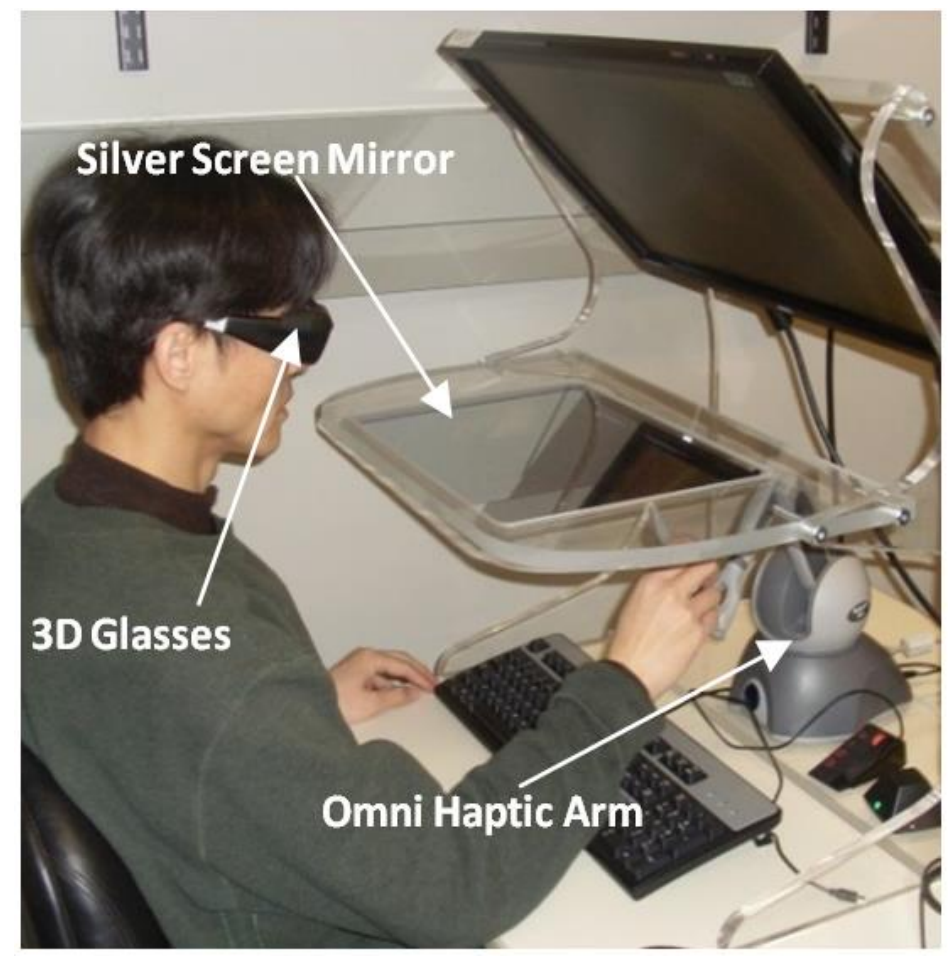

Figure 2.1 Hardware for myringotomy simulator. In the DevinSense Display 300 system (Devinsense Display Solutions, Sundbyberg, Sweden), scenes are viewed using 3D glasses. To the user, the 3D scene appears in the space below the silver screen mirror. The scene in this region is correctly collocated with the haptic arm so movements of the haptic arm appear to occur in the same space as the 3D scene.

Unity3D, a leading commercial digital gaming engine, was used as the framework for the new simulator, however custom code had to be written using the C\# programming language to appropriately model and manipulate the eardrum and instruments. A functional block diagram of the simulation software is shown in Figure 2.2. Unity has built-in support for Nvidia's PhysX physics calculation engine and for the Direct3D (Microsoft Corp, Redmond, WA) and OpenGL (Khronos Group, Beaverton, OR) graphics rendering engines in Windows. Digital models of the auricle, ear canal, eardrum, middle-ear space, and surgical tools were imported into Unity3D. Most of the program 
code was added into two built-in functions, Update and FixedUpdate, to control the behaviors of each imported digital model and to manipulate the rendering and physics engines.

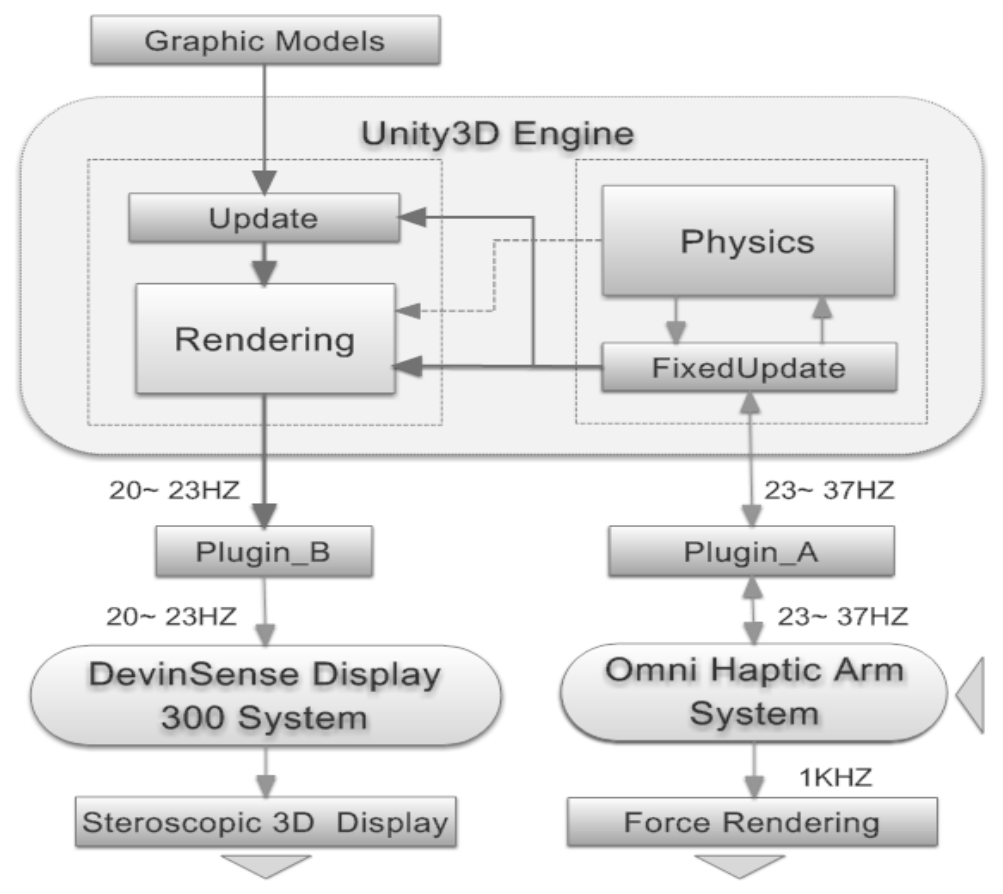

Figure 2.2 Simulation software's framework. See text for description. The box labelled "Physics" represents the physics calculation engine in Unity3D. The box labelled "Rendering" represents graphic rendering engines in Unity3D. The boxes labelled "Update" and "FixedUpdate" represent built-in functions in Unity3D. The box labelled "Graphic models" stands for the digital models imported into Unity3D for the simulation. The box labelled "Plugin_A" is the plugin for integration of the Omni haptic arm with Unity3D, whereas the box labelled "Plugin_B" is the plugin for 3D stereo display in Unity3D.

Unity3D does not have native support for haptic devices (e.g., Phantom Omni) or for stereoscopic 3D displays. Therefore, the basic Omni application programming interface (API) for force rendering and positioning provided by the manufacturer forms the basis for "Plugin_A" shown in Figure 2.2 [21]. The plugin is embedded into the myringotomy 
simulator to integrate the Omni to Unity3D for positioning and force rendering via the box labelled "Plugin_A" shown in Figure 2.2. In order to allow a stereo 3D display, "Plugin B" was imported into the simulator as seen in the same Figure 2.2 [22].

\subsubsection{Geometrical model}

A high-resolution 3D clinical computed tomography (CT) image was used to reconstruct the geometric model of the ear canal. The auricle, ear canal, eardrum, ossicles and middle-ear walls were all modeled, as these structures are relevant to myringotomy. Although surgery is only performed on the eardrum, the blade must be navigated down a narrow ear canal without creating any trauma or bleeding. Inclusion of the ossicles and middle-ear wall behind the eardrum was necessary as these structures can accidentally be damaged during myringotomy. 


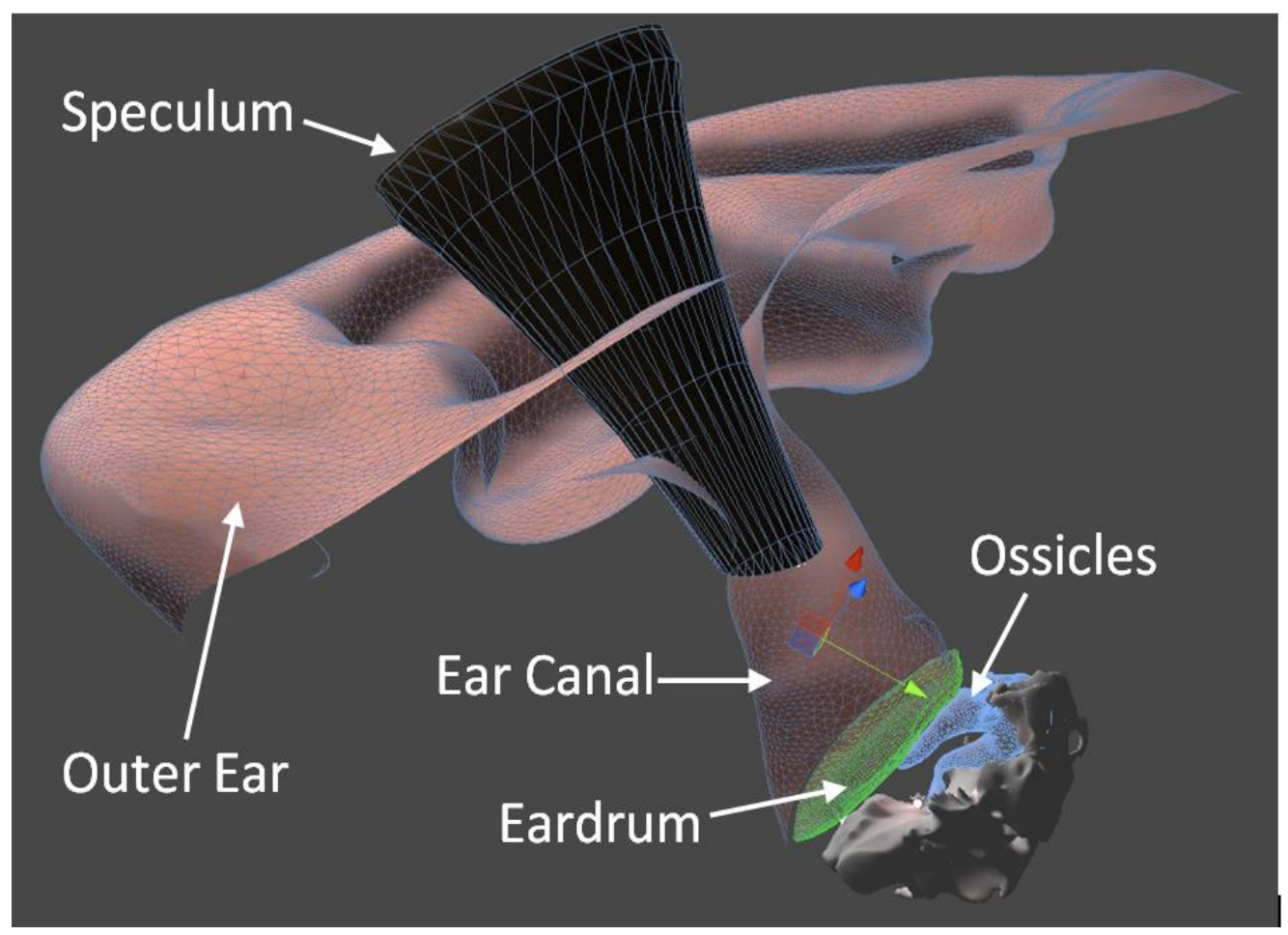

Figure 2.3 Geometrical model of the ear and speculum. For ease of viewing, the middleear wall is not shown. Users of the system would not see the underlying meshes. Figure 2.10 shows what the user would see with the exception of stereo viewing.

All structures were manually segmented by a fellowship-trained otologist (SKA) using the MIPAV (Medical Image Processing, Analysis, and Visualization) software that is freely available from the National Institutes for Health (Bethesda, MD). By applying the Marching Cubes algorithm implemented in MIPAV, the contours for each structure were converted into a triangulated surface mesh. The meshes were imported into Geomagic Studio (Geomagic, Inc., Morrisville, NC) to smoothen the surfaces and create the final model of the ear to be used with Unity3D (Figure 2.3). The virtual surgical tools such as blade, forceps, tube, and speculum were created using the Blender software (Blender.org, Amsterdam, The Netherlands) and imported into Unity3D. 


\subsubsection{Deformation and collision models for the eardrum}

To simulate the deformation of the eardrum, a mass-spring system was created (Figure 2.4) using the eardrum model developed in Section 2.2.2. Prior to incorporation into the simulation software, the eardrum mesh in Section 2.2.2 was decimated so that it consisted of 700 nodes and 1250 triangles. The size of the mesh was a compromise between accuracy and real-time performance for collision detection, deformation modeling, cutting simulation and interactive visualization. Each node in the mesh is regarded as a point mass. To model deformations in Unity3D, an invisible rigid ball was placed at each node. The radius of the ball was slightly smaller than half of the distance between adjacent nodes, and the balls were connected to each other using a "Spring Joint", a Unity3D component that groups together two rigid bodies and constrains them to move as if they are connected by a spring. The mass of each ball was set to a very small value because inertial effects can be ignored for the slow movements made during surgical procedures. The "Spring Joint" also has a damping coefficient and a spring stiffness associated with it. The mechanical behavior of the virtual eardrum is mainly governed by the spring constants used in modeling. Initially, the spring constants were determined using van Gelder's formulation [23] which requires membrane thickness and Young's modulus. The eardrum was taken to have a uniform thickness of $75 \mu \mathrm{m}$ and a Young's modulus of $40 \mathrm{MPa}$ as in previous modeling studies [24]. The spring constants were subsequently fine-tuned by surgical collaborators to match their subjective impressions in the operating room.

The balls along the boundary of the spring network were fixed to simulate the physiological condition of the actual eardrum being attached to solid bone along its edges. 
The attachment of the eardrum to the malleus (the most lateral ossicle) was simulated by constraining the motion of the balls along the central portion of the eardrum to simulate the loading of the eardrum at its center.

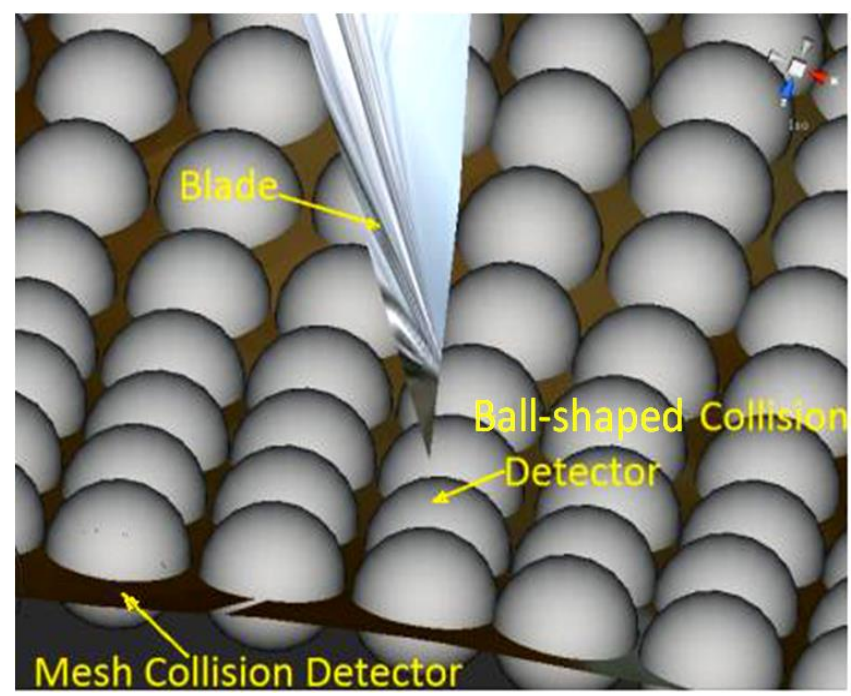

Figure 2.4 Collision models. Ball-shaped collision detectors are associated with each node of the eardrum model and control eardrum deformation. The mesh collision detector activates cutting when contacted by the virtual blade.

To implement collision detection in Unity3D, two types of collision detectors were designed for the eardrum: a ball-shaped collision detector and a mesh collision detector (Figure 2.4). The ball-shaped detector is associated with every ball of the mass-spring network. When the blade touches a ball, the ball will move and lead to a deformation of the entire eardrum mesh. The mesh collision detector is placed just underneath the surface of the eardrum mesh, as shown in Figure 2.4, and it is used to activate the cutting algorithm. While the ball-shaped detectors are responding dynamically and causing deformations, the mesh collision detector is immediately being updated and transformed to ensure that it stays aligned with the deformed eardrum mesh. 
During the simulated operation, if the blade touches a ball before penetrating the eardrum mesh, it causes a local deformation providing an important visual cue that the eardrum has been touched. When the blade moves further, the balls are pushed away, and the blade touches the eardrum mesh collision detector and activates the cutting algorithm. During the cutting, the blade interacts with the ball-shaped collision detector and the eardrum mesh collision detector at the same time. The balls along the cutting path successively collide with the blade causing progressive deformation of the eardrum mesh; meanwhile, the incision path is calculated when the blade touches the eardrum mesh collision detector.

\subsubsection{Cutting algorithm}

The subdivision cutting (SC) algorithm [25] was implemented for cutting because it accurately represents the actual cutting curve. Briefly, the basic SC approach splits the triangles along the sampled cutting path. Note the distinction between "actual cutting curve" and "sampled cutting curve": the "actual cutting curve" reflects the physical motion of the virtual blade, whereas the "sampled cutting curve" is a digitized representation of the actual cutting curve. The sampled curve is formed by computing the intersection points between the virtual blade and the triangulated surface representing the eardrum. For instance, in Figure 2.5-a, the blue curve is the actual cutting curve, whereas polyline ABCDEFG is the sampled cutting curve formed by all collision points between the virtual blade and the edges of the triangles representing the surface. After computing intersections, the algorithm splits each triangle in the original triangular mesh so that edges along the sampled cutting curve form sides of new triangles. For example, triangle $\mathrm{LMN}$ in the original mesh (see Figure 2.5-a) is split by segment $\mathrm{AB}$ and re- 
meshed by shaded triangles 1 (formed by vertices LMA), 2 (vertices LAB), and 3 (vertices BAN) as shown in Figure 2.5-b.
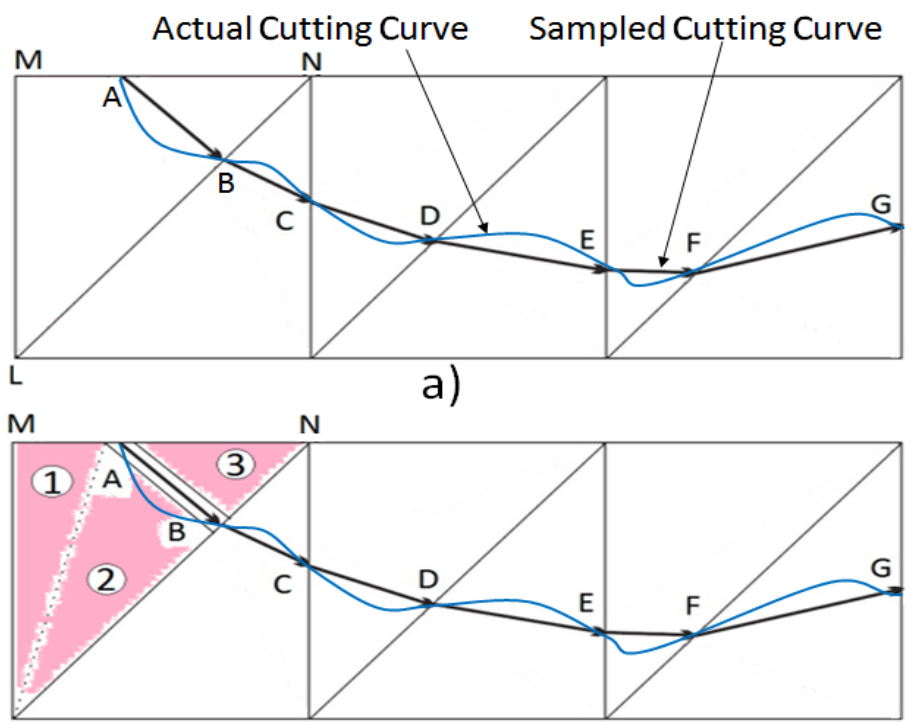

b)

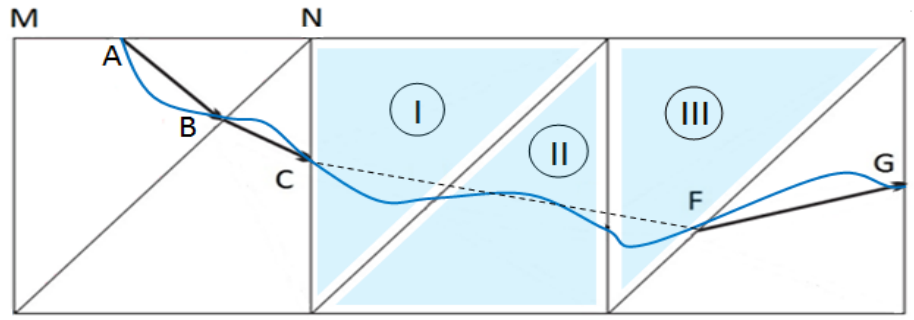

c)

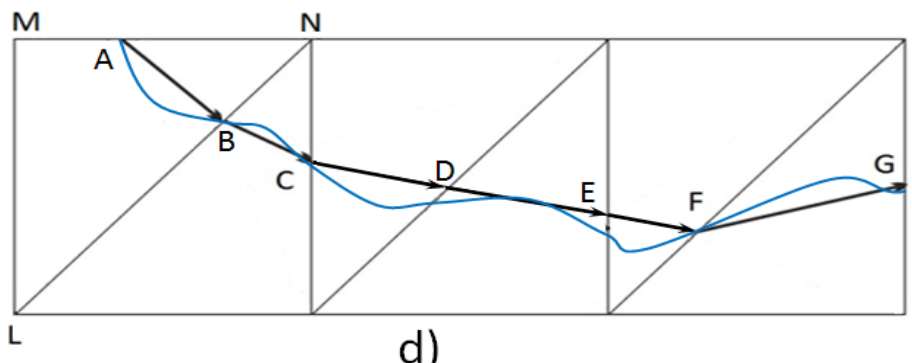

d)

Figure 2.5 Illustration of subdivision cutting. a) An illustration of the actual cutting curve (blue) and sampled cutting curve (black). b) The triangle $L M N$ is split into two parts and re-meshed by triangles 1 (nodes $L M A$ ), 2 (nodes $L A B$ ) and 3 (BAN). c) Three shaded triangles are missed in the sampled cutting curve due to an excessively fast cutting speed. Dashed line CF indicates the vector that the cutting plane goes through. d) Polyline $A B C D E F$ is the "corrected" sampled cutting curve. 
The number of collision points calculated along the actual cutting curve is related to the cutting speed. If the mesh triangles are small, even at normal cutting speed, it always occurs that more than one triangle is contained between a pair of successive points along the sampled cutting curve. In the example shown in Figure 2.5-c, assume the sampled cutting curve is polyline ABCFG. $\mathrm{C}$ and $\mathrm{F}$ are a pair of successive points along the polyline. The points D and E that are present in Figure 2.5-a are not included since there were no collision points calculated between $\mathrm{C}$ and $\mathrm{F}$ (due to the excessively fast motion of the virtual blade). The shaded triangles I, II, and III are nested between C and F, but they need to be split. To solve this situation, a cutting plane is used to intersect these nested triangles in one shot rather than splitting every contained triangle by invoking the basic SC algorithm recursively as described in reference [25]. This cutting plane is determined by the coordinates of the point $\mathrm{C}$ and $\mathrm{F}$ and a normal vector $\mathrm{n}$, where $\mathrm{n}$ is defined as:

$$
\mathbf{n}=\mathbf{C F} \times(\mathbf{V C}+\mathrm{VF}) / 2
$$

where $\mathrm{VC}$ and $\mathrm{VF}$ are two unit vectors representing the axis of the blade at point $\mathrm{C}$ and at point $\mathrm{F}$, and $\mathbf{C F}$ is a unit vector pointing from $\mathrm{C}$ to $\mathrm{F}$. Figure 2.5 -d shows the "corrected" version of $2-5 \mathrm{c}$.

\subsubsection{Speculum and microscope}

Before the cutting is performed, the surgical view can be adjusted by rotating the speculum and tilting the microscope. In this simulator, the movements of the speculum and of the microscope are simulated by moving the handle of a haptic arm in 3D space. 
The simulated microscope was designed to move in a small bounded box. The user can feel friction effects when moving the microscope within the box, but the movement is completely limited by haptic feedback at the edge of the box to simulate a rigid boundary. Friction is modeled by Plugin_A (see Figure 2.2 and Section 2.2.1) by a parameter normalized between 0 to 1.0 . The value of this parameter was set empirically by an experienced ear surgeon. The zoom operation on the virtual microscope is available at any stage of the simulated surgery.

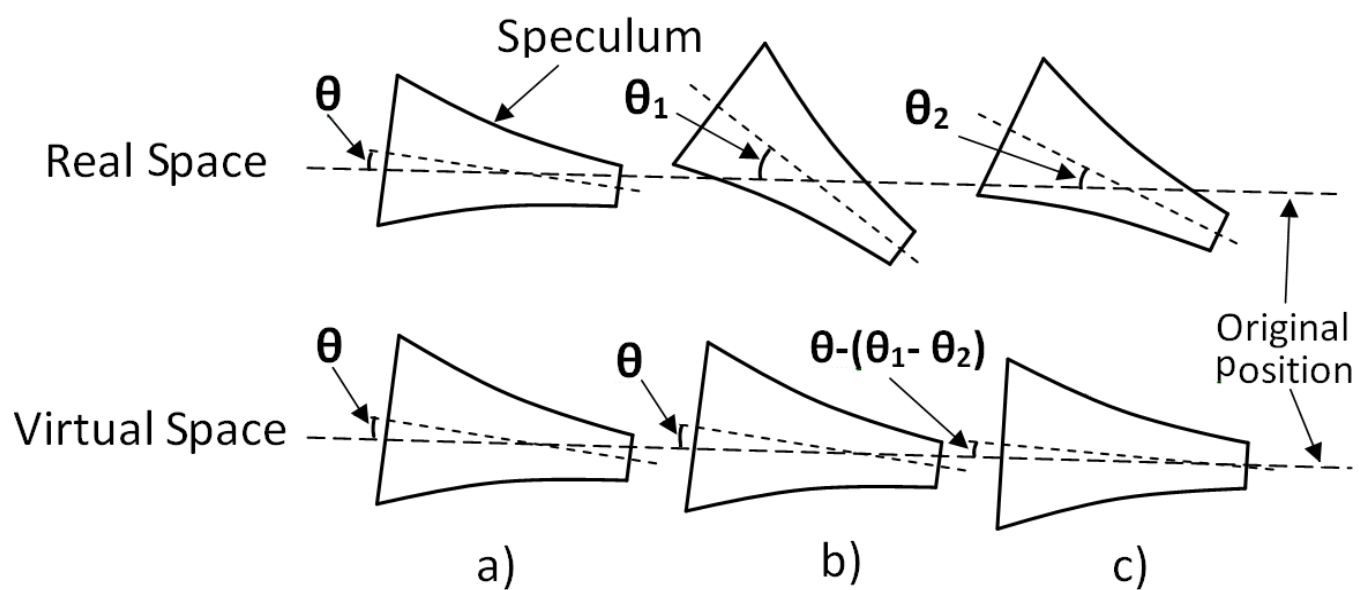

Figure 2.6 Rotation of the speculum. The top panel illustrates three rotation values that the speculum is taken through in real space. The bottom panel indicates three rotation values in the virtual scene that are actually rendered. $\theta$ is the rotational limit based on ear canal geometry. In the top panel (real space), the user can rotate the haptic arm to $\theta 1>\theta$, but in the corresponding virtual scene (bottom panel), the rotation is limited to $\theta$. When the user attempts to decrease the amount of rotation to $\theta 2(\theta<\theta 2<\theta 1)$ in real space, the rendered angle in the virtual scene is $\theta-(\theta 1-\theta 2)$.

Along with the microscope, the user can also rotate the virtual speculum around a selected pivot to see different parts of the eardrum. The position of the pivot is fixed by force feedback to simulate the speculum resting within the ear canal. Since the ear canal is essentially a narrow cylinder, this greatly limits the rotation of the speculum. In the 
corresponding virtual scene, the rotation of the speculum is limited within a very small range, for example, within $\theta$ as shown in Figure 2.6-a (top and bottom panels). The value $\theta=5$ degrees was used based on the geometry of the ear canal. In the simulation, the rotation of the speculum is controlled by the handle of the haptic arm, but the Omni haptic arm is not able to provide torque to the handle to restrain rotation beyond $\theta$. Therefore, the rotation of the haptic arm cannot be limited in real space. In the implementation, while the haptic arm rotates beyond the limit $(\theta)$ to $\theta 1(\theta 1>\theta)$ as shown in Figure 2.6-b (top panel), the rotation of the virtual speculum does not follow the change and is stopped automatically at $\theta$, as shown in Figure 2.6-b (bottom panel). When the rotation direction is changed and the rotation angle decreases from $\theta 1$ to $\theta 2$ with $\theta<$ $\theta 2<\theta 1$ as shown in Figure 2.6-c (top panel), the virtual speculum will start to rotate back, from $\theta$ to $\theta-(\theta 1-\theta 2)$ as shown in Figure 2.6-c (bottom panel). This algorithm lets the user easily recover control of the virtual speculum lost due to the excessive rotation of the haptic arm beyond the limit $\theta$ in real space, and thus can effectively maintain instant interaction between the user and the virtual speculum.

\subsubsection{Tube insertion}

After the incision is made (Figure 2.7-a), surgeons usually use a suction tip inserted into the incision to drain fluid from the middle ear. In the simulator, the suction tip is modeled as a rigid body. When the suction tip squeezes into the incision and touches the ballshaped colliders around the incision, these invisible rigid balls are pushed away and move in opposite directions as shown in Figure 2.7-b. This leads to an increase in the distance between mesh nodes that are attached to the centers of these balls and are situated 
opposite each other across the incision. The displacements of the nodes along the cutting path simulate splaying of the incision as shown in Figure 2.7-c.
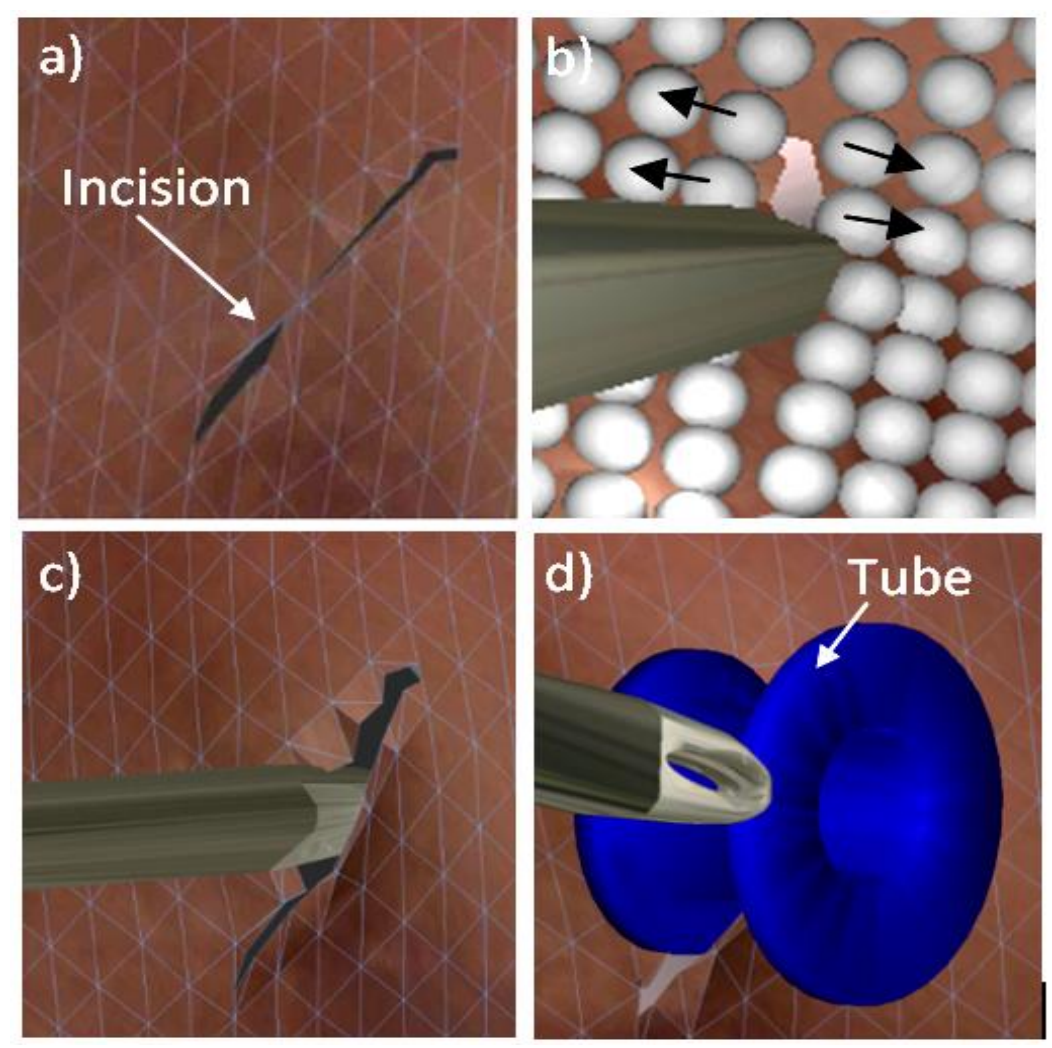

Figure 2.7 Illustration of tube placement. a) The incision shape after cutting. b) The invisible rigid balls are pushed away to widen the incision. c) The incision is deformed by a suction device. d) The incision is deformed by positioning of a tube.

Next, the surgeon will grasp a ventilation tube using virtual forceps and navigate the tube down the ear canal to the incision in the eardrum. The movement of the virtual forceps is controlled by moving the haptic arm in 3D space. When grasping a ventilation tube, the opening and closing actions of the forceps are controlled by pressing a button on the handle of the haptic arm. Both the forceps and the tube are modeled as rigid bodies, so the grasping action does not deform either item. The physical interaction between the 
forceps and the tube is ignored in the simulation for two reasons. First, the Omni does not have a grasping end effector. Secondly, the force needed to grasp the tube is very small; therefore, the tube is typically grasped and attached rigidly to the forceps without dropping. If the trainee prematurely lets go of the grasping button, an animation causes the tube to fall based on gravity.

After moving the tube down the ear canal, the user will attempt to insert the virtual tube into the incision. This will deform the incision and the eardrum. The same method discussed above is applied to simulate splay of the incision when the tube enters the incisions as shown in Figure 2.7-d, and the interactions between the tube and eardrum are based on the eardrum physics model that was discussed previously in Section 2.2.3. After insertion, the user can use a simulated needle to adjust the tube into the final position. When the tube is touched by the needle, it rotates and slides into the incision along a pre-calculated route.

\subsection{Results and discussion}

\subsubsection{System performance analysis}

In Unity3D, each object can be attached with more than one script. These scripts describe the behaviors of the graphical objects or respond to events from the scene or call an API (e.g., that associated with the haptic arm). Each script includes two built-in functions: "Update" and "FixedUpdate". Update is called when the game engine renders every graphical frame. The graphical frame rate (FR) is automatically changed by Unity3D at runtime depending on loading of the CPU. Physics calculations such as collision detection are performed in discrete fixed time steps that are not dependent on the 
graphical frame rate. The value of the Fixed Timestep (FTS) is a time interval set by the programmer in Unity3D and dictates when physics calculations are done. It is very important for stable physics simulation as discussed below. FixedUpdate is called when each physics calculation step is executed. The relationships of all above modules are illustrated in Figure 2.2.

At runtime, the kinematic time-series data of the haptic arm (positions and rotations as a function of time) are read within the FixedUpdate function by calling Plugin_A. These data are then smoothed by applying a moving average filter. A filter of length 4 for rotation was empirically determined as a reasonable compromise between real-time performance and vibration reduction from the haptic arm; no filtering was required for translations. After processing these input data in FixedUpdate, force rendering commands are sent to the Omni's device driver by invoking Plugin_A. Then the driver renders forces at a high frequency of $1 \mathrm{kHz}$. The data related to movements of the objects are used directly for graphical rendering at a rate of FR. The eardrum mesh is dynamically refreshed within the Update function for displaying, and the myringotomy cutting algorithm is applied on the eardrum mesh in the meantime.

Theoretically, the smaller the value of FTS, the higher the frequency with which FixedUpdate is called. This will contribute to more accurate physics simulation and more smooth and coherent force feedback. However, high frequency physics calculations usually dramatically increase CPU load and have negative impact on real-time performance. In this simulator, eardrum deformation and cutting modeling heavily depend on physics calculations. High frequency physics calculations inevitably result in low FR. 


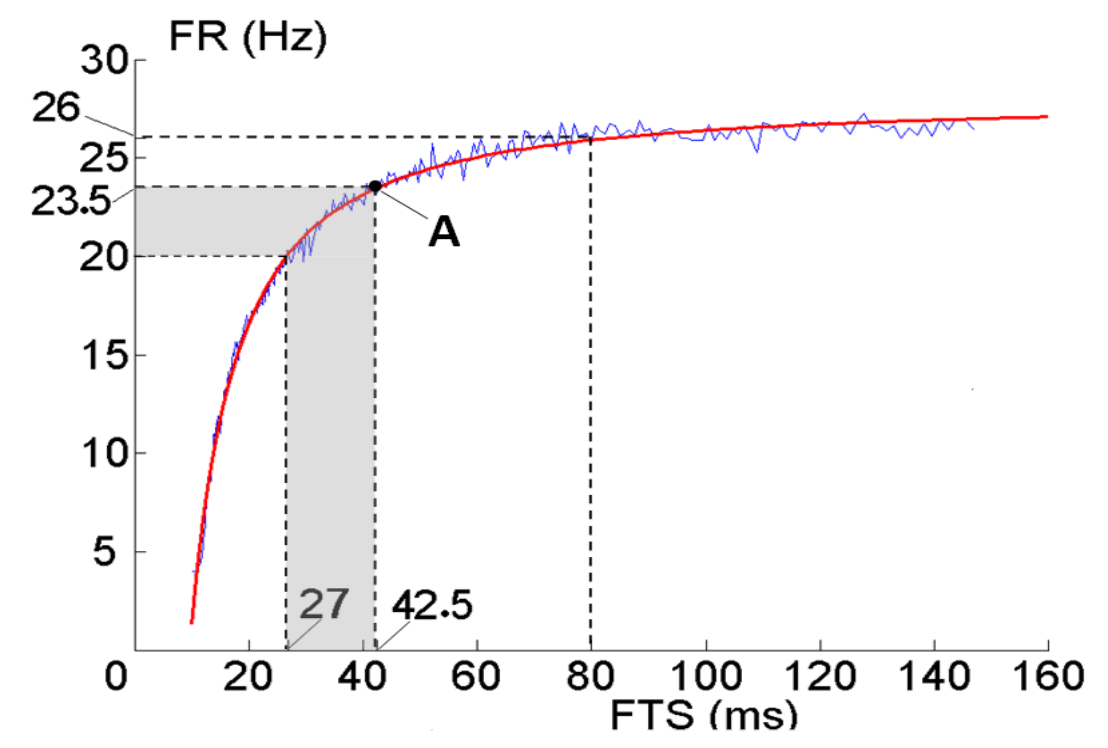

Figure 2.8 Relationship of FR and FTS. FR is graphic rendering frame rate. FTS is the "Fixed Timestep" for physics calculations defined by Unity3D. The blue curve represents the raw data, whereas the red curve is a least-squares fitted exponential to the data. FTS values between $27 \mathrm{~ms}$ and $42.5 \mathrm{~ms}$ result in accurate physics calculations and also result in $\mathrm{FR}$ values $(20 \mathrm{~Hz}$ to $23.5 \mathrm{~Hz}$ ) that lead to a smooth animation.

To maintain a balance between FR and the physics calculation frequency which is equal to the inverse of FTS, i.e., FTS-1, a test is performed on the simulator. The myringotomy simulator runs beginning at a small pre-set FTS value of $10 \mathrm{~ms}$ (i.e., physics calculation frequency of $100 \mathrm{~Hz}$ ). Then this value is gradually increased automatically, and the corresponding FR values are recorded. The results are shown in Figure 2.8. As FTS increases, FR increases rapidly at first then increases more gradually with increasing FTS until FR plateaus at about $26 \mathrm{~Hz}$ with further increases in FTS above $80 \mathrm{~ms}$. From the figure, it can be seen that a small FTS value of $10 \mathrm{~ms}$ results in a very small FR value of $5 \mathrm{~Hz}$. Although such a small value of FTS would result in accurate physics calculations, the resulting slow FR is not enough to support smooth graphical rendering of movements. However, increasing FTS beyond $80 \mathrm{~ms}$ which would result in inaccurate physics 
calculations, produces negligible benefits in FR. At the point A in Figure 2.8, FR (23.5 $\mathrm{Hz})$ is equal to the physics calculation frequency $[(0.0425 \mathrm{~s})-1=23.5 \mathrm{~Hz}]$. As FTS is increased beyond $42.5 \mathrm{~ms}$, the physics calculation frequency was empirically deemed to be too low in terms of accuracy of the physics calculations and force feedback. So FTS values above $42.5 \mathrm{~ms}$ are not suitable for myringotomy simulation in which the realism of tissue deformation heavily depends on physics calculations. Practically, FTS values between $27 \mathrm{~ms}(37 \mathrm{~Hz})$ and $42.5 \mathrm{~ms}(23.5 \mathrm{~Hz})$ are appropriate as empirically determined by surgeons and residents who tried the simulator; the corresponding FR values range from $20 \mathrm{~Hz}$ to $23.5 \mathrm{~Hz}$. In this range, which is shaded in Figure 2.8, a stable physics simulation is achieved with accurate force feedback and smooth display.

\subsubsection{Cutting performance}

As mentioned in Section 2.2.4, a cutting plane is used to split missed triangles along the cutting path when the cutting speed is too fast. The use of a cutting plane as opposed to recursive calls of the SC algorithm reduces the calculation time to deal with missed triangles with the intent of meeting the need for real-time performance; however, the smoothness of the rendered cutting path may be compromised. To test the algorithm, cutting is performed by a programmed virtual blade with different speeds along the same cutting curve, i.e., the blade was not user controlled but was programmed to follow a specific test trajectory at a variety of preset speeds. The object to be cut is the virtual 3D eardrum illustrated in Figure 2.9. 


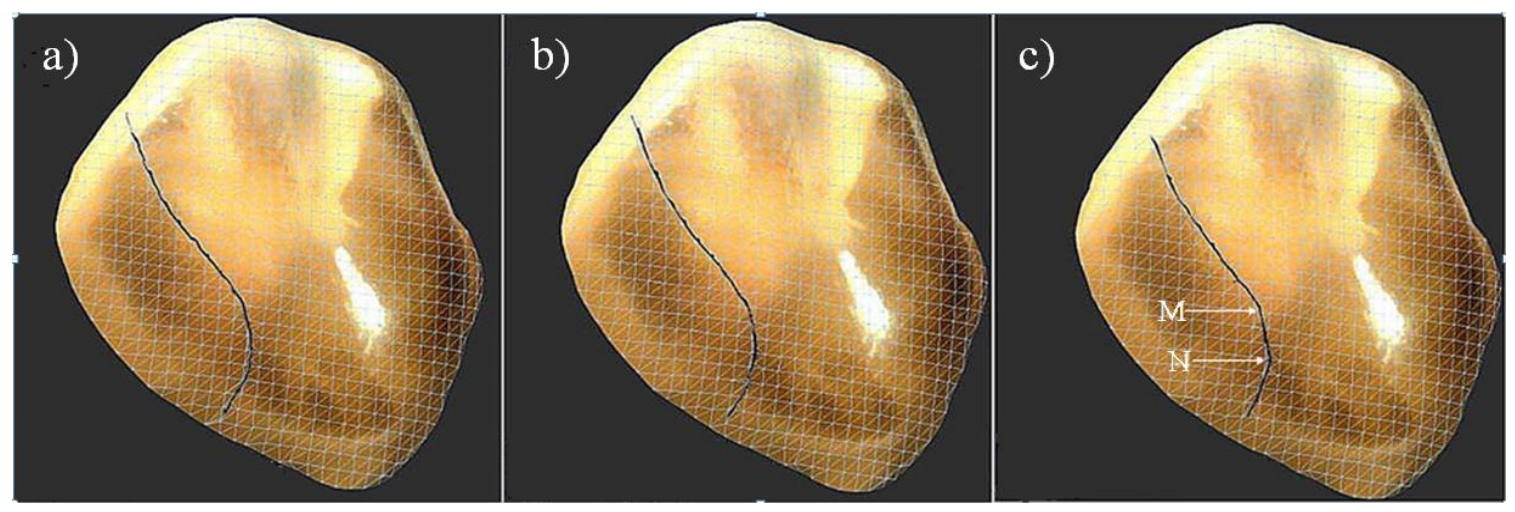

Figure 2.9 Effects of blade movement speed on cutting algorithm. (a) Cutting is performed with a slow cutting speed denoted as $V$. Total cutting time is 9.65 seconds. (b) Cutting is performed at speed of $9.75 \mathrm{~V}$ with total cutting time of 0.99 seconds (c) Cutting is performed at a fast speed of $14.8 \mathrm{~V}$ with a total cutting time of 0.65 seconds.

In Figure 2.9, panels (a)-(c) illustrate the performance of the algorithm with progressively increasing cutting speeds from left to right. Even with over a 10-fold increase in cutting speed the improved SC algorithm easily handles the increased speed and maintains a reasonably smooth cutting path. However, only one difference can be seen between panel (a) [slowest cutting speed] and panel (c) [fastest cutting speed]: The cutting path in (c) becomes a little bit sharper relative to the path in (a) where the blade turns, e.g., points labelled $\mathrm{M}$ and $\mathrm{N}$ in (c). This happens because as the cutting speed increases, the distance between two sampled points becomes longer, and the path will be arbitrarily interpolated using the cutting plane rather than following the actual cutting curve.

\subsubsection{Overall impression of simulator}

A complete simulator has been now implemented, with the major graphical components shown in Figure 2.10-a. The user experiences immersive interactions with virtual objects through stereo 3D vision. Different parts of the eardrum can be observed by rotating the 
virtual speculum and positioning and orienting the microscope as shown in Figure 2.10-b. After the view of the microscope is selected, cutting is performed on the deformable eardrum as shown in Figure 2.10-c. During the cutting, deformation of the eardrum is observed in real time. During tube insertion, the user sees the incision splaying and the tube entering as shown in Figure 2.10-d. Then the tube is pushed into the incision appropriately by a needle and repositioned to sit within the incision; the final position of the tube is shown in Figure 2.10-e. 

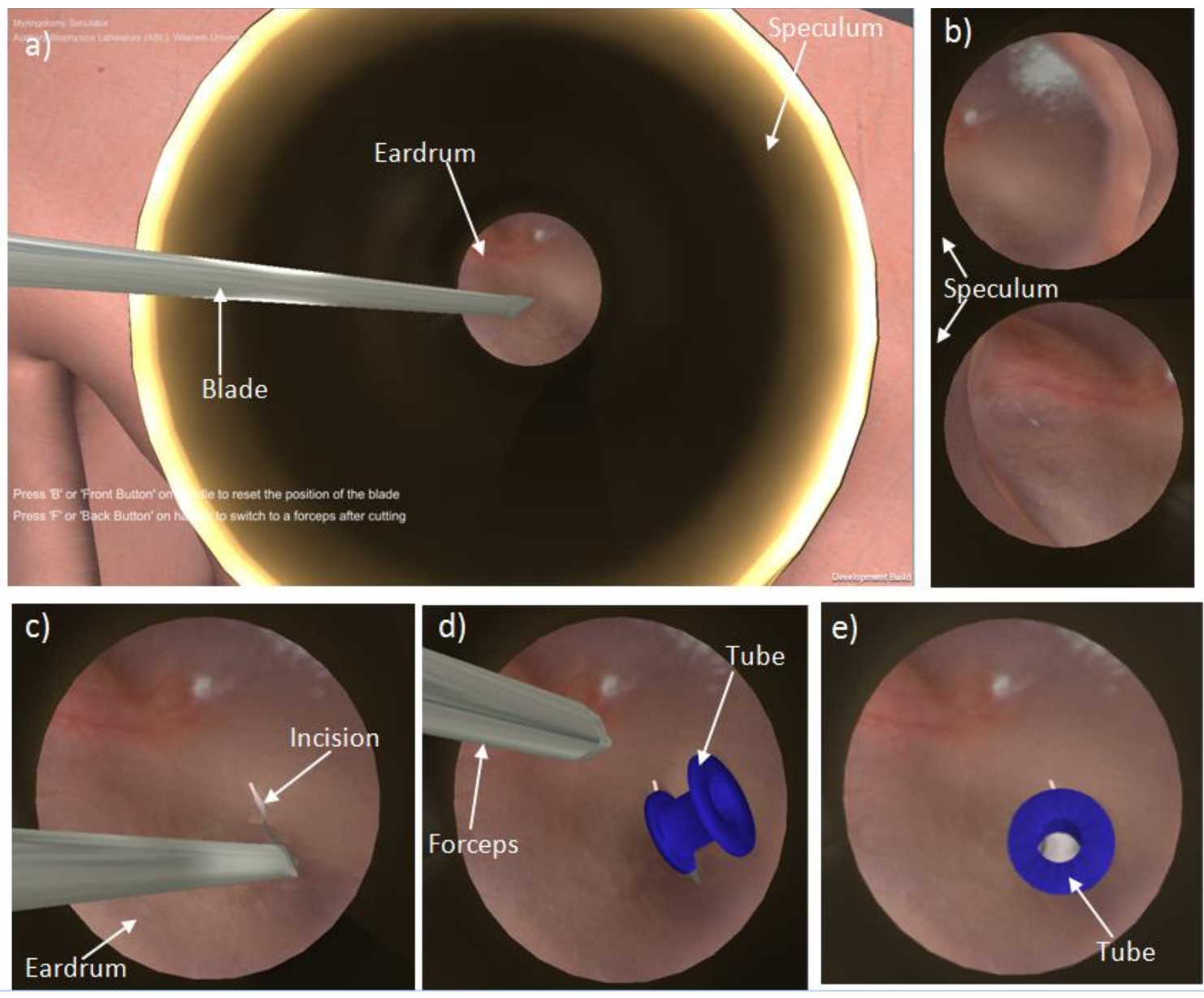

Figure 2.10 Simulated scene as viewed by user. Excludes stereo display. a) Zoomed out view. b) Zoomed in view demonstrating viewing of two different parts of the model eardrum by rotating the virtual speculum or tilting the simulated microscope. The black areas are portions of the speculum through which the eardrum is seen. c) Demonstration of cutting. d) Demonstration of tube insertion. e) The tube in its final position.

Quantitative performance tests in Sections 2.3.1 and 2.3.2 demonstrate that all operations can be performed in real time. Although the simulated microscope, speculum, and blade can all be manipulated individually, in an actual procedure, combinations of these instruments need to be manipulated simultaneously. In consultation with instructing surgeons at Western University, it was felt that in the first iteration of the design, separating the microscope movement, speculum movement, and blade navigation may 
suffice for skills transference for junior residents. Certainly, low fidelity simulators that train on specific simplified tasks can often be equally efficacious to high fidelity simulators [26]. A skills transference study is being planned to determine the adequacy of our design choices.

Separating surgical tasks as noted above greatly simplified programming and hardware requirements. As an example, one might consider the task of navigating a myringotomy blade through a speculum and down the ear canal towards the eardrum. During an actual procedure, the surgeon holds the speculum with one hand and navigates the blade using the other hand. To steady the blade, the surgeon rests it against a portion of the speculum. Learning to position and orient a speculum and to simultaneously insert a blade in the speculum while maintaining an unobstructed view of the operative site is challenging. The current simulator cannot be used to train on this aspect of the procedure. Creating a virtual speculum on which a virtual myringotomy blade can rest is a difficult engineering task. It would require real-time multi-point collision detection along the length of the blade, as well as a 6-DOF haptic arm in order to provide the necessary force feedback. Our future goal is to create a hybrid simulator where an actual myringotomy blade is attached to a haptic arm and navigated through a real speculum (that has been registered to the virtual scene). A second haptic arm would monitor the movement of the speculum that has been placed into a mannequin head. The trainee would then feel a real blade, navigate it through a real speculum, be able to use the speculum as a fulcrum, and finally be able to move the speculum and the blade simultaneously. In the present study, the primary objective was to integrate various components of the simulator using off-the- 
shelf interfaces and a digital gaming software engine, and simplifications were made to at least demonstrate feasibility.

Qualitative feedback from instructing ear surgeons indicates that the models are anatomically accurate and contain all landmarks necessary for surgical training. Moreover, surgeons agreed that speculum placement, microscope positioning, eardrum cutting and tube insertion as simulated could potentially aid in training on individual tasks. However, an extensive face and content validity study is planned to quantify these impressions. It is hoped that construct validity can then be established after incorporating performance metrics to differentiate novices from experts. A final goal is to perform a rigorous transference study to determine whether this VR training translates into improved OR (operating room) performance.

The current implementation of the simulator incorporates only one ear. Repeated training with the same model is problematic because users could adapt to the particular anatomy being simulated. In practice, surgeons must deal with large anatomical variations between patients. Incorporation of other ears in the simulator is simple in terms of programming, but the main challenge is constructing models from medical images. Specifically, manual delineation of anatomical structures from images as described in Section 2.2.2 is both tedious and time consuming, hence we are investigating semi-automated delineation approaches such as active contours, i.e., contours that change shape automatically to fit boundaries in images [27]. When using active contours, the user only needs to draw an anatomical boundary approximately in one image slice. After the contour has been automatically deformed by the active contour algorithm to fit anatomical boundaries in the image, it can be propagated to the next image slice to initiate automated boundary 
fitting until all image slices have been processed. Our group has previously demonstrated this approach for other anatomical structures [28].

Despite positive qualitative feedback from end users, several other areas of improvement were noted. Currently, grasping of the tube is not simulated but is rendered as a preset animation. This was done because the haptic arm utilized in this work does not have a grasping end effector. However, such end effectors have been implemented for more costly haptic devices [29]. Moreover, the inclusion of performance metrics and tutoring based on automated error detection are recommended. As noted, the inclusion of performance metrics would pave the road for a discriminant validity study to determine if the simulator and its metrics can distinguish novices from experts.

\subsection{Conclusions}

The VR-based myringotomy simulator implemented in this work can allow the user to perform individual tasks such as speculum and microscope adjustment, blade navigation, cutting of the eardrum, and tube placement. The cutting simulation can be performed over a wide range of speeds from slow to unrealistically fast while maintaining a visually smooth cutting path. System performance analysis confirms that simulations operate at interactive rates. A key contribution of this work is implementation using a widely available digital gaming software engine that is cost effective and that is well maintained. Initial opinions from surgeons involved in the design of this simulator are encouraging, and planning of rigorous studies to evaluate face, content and construct validity as well as skills transference is underway. 


\subsection{References}

1 M. Montague, M. S. W. Lee, S. S. M. Hussain, "Human error identification: an analysis of myringotomy and ventilation tube insertion," Archives of Otolaryngology -Head and Neck Surgery, vol. 130, no. 10, pp. 1153-1157, 2004.

2 S. Weghorst, C. Airola, P. Oppenheimer, C. V. Edmond, T. Patience, D. Heskamp, J. Miller, "Validation of the Madigan ESS simulator," Studies in Health Technology and Informatics, vol. 50, pp. 399-405, 1998.

3 S. M. Anil, Y. Kato, M. Hayakawa, K. Yoshida, S. Nagahisha, T. Kanno, "Virtual 3dimensional preoperative planning with the dextroscope for excision of a 4th ventricular ependymoma," Minimally Invasive Neurosurgery, vol. 50, pp. 65-70, 2007.

4 M. Audette, H. Delingette, A. Fuchs, O. Astley, K. Chinzei. "A topologically faithful, tissue-guided, spatially varying meshing strategy for computing patient-specific head models for endoscopic pituitary surgery simulation." Studies in Health Technology and Informatics, vol. 119, pp. 22-27, 2006.

5 B. Tolsdorff, A.Pommert, K.H. Höhne, A.Petersik, B. Pflesser, U. Tiede, R. Leuwer, "Virtual reality: a new paranasal sinus surgery simulator," Laryngoscope, vol. 120, pp. 420-426, 2010.

6 G. J. Wiet, D. Stredney, D. Sessanna, J. A. Bryan, D. B. Welling, P. Schmalbrock, "virtual temporal bone dissection: an interactive surgical simulator," Otolaryngology -Head and Neck Surgery, vol. 127, pp. 79-83, 2002.

7 D. Morris, C. Sewell, F. Barbagli, K. Salisbury, N. H. Blevins, S. Girod, "Visuohaptic simulation of bone surgery for training and evaluation," IEEE Computer Graphics and Applications, vol. 26, pp. 48-57, 2006.

8 C. Sewell, D. Morris, N.H. Blevins, S. Dutta, S. Agrawal, F. Barbagli, K. Salisbury, "Providing metrics and performance feedback in a surgical simulator," Computer Aided Surgery, vol. 13, pp. 63-81, 2008.

9 A. Arora, S. Khemani, N.Tolley, A. Singh, J. Budge, D.A. Varela, H.W. Francis, A. Darzi, N.I. Bhatti, "Face and content validation of a virtual reality temporal bone simulator," Otolaryngology-Head and Neck Surgery, vol. 146, pp. 497-503, 2012.

10 P. Hong, A. N. Webb, G. Corsten, J. Balderston, R. Haworth, K. Ritchie, E. Massoud. "An anatomically sound surgical simulation model for myringotomy and tympanostomy tube insertion," International Journal of Pediatric Otorhinolaryngology, vol.78, pp. 522-529, 2008.

11 T. Walker, S. Duvvi, B. N. Kumar, "The Wigan Grommet Trainer," Clinical Otolaryngology, vol. 31, no. 4, pp. 349-350, 2006.

12 M. Duijvestein, J. Borgstein, "The Bradford Grommet Trainer," Clinical Otolaryngology, vol. 31, no. 2, pp. 163-163, 2006.

13 A. Leong, S. Kundu, P. Martinez-Devesa, C. Aldren, "Artificial ear: a training tool for grommet insertion and manual dexterity," ORL : Journal for Oto - Rhino Laryngology and Its Related Specialties, vol. 68, no. 2, pp. 115-117, 2006.

14 L. J. Sowerby, G. Rehal, M. Husein, P. C. Doyle, S. Agrawal, H. M. Ladak, "Development and face validity testing of a three-dimensional myringotomy 
simulator with haptic feedback," Journal of Otolaryngology: Head and Neck Surgery, vol. 39, no. 2, pp. 122-129, 2010.

15 B. Wheeler, P. C. Doyle, S. Chandarana, S. Agrawal, M. Husein, H. M. Ladak, "Interactive computer-based simulator for training in blade navigation and targeting in myringotomy," Computer Methods Programs in Biomedicine, vol. 98, no. 2, pp. 130-139, 2010.

16 A. K. Ho, H. Alsaffar, P. C. Doyle, H. M. Ladak, S. K. Agrawal, "Virtual reality myringotomy simulation with real-time deformation: development and validity testing," The Laryngoscope, vol. 122, no. 8, pp. 1844-1851, 2012.

17 S. Marks, J. Windsor, B. Wünsche, "Evaluation of game engines for simulated surgical training," Proceedings of the 5th International Conference on Computer Graphics and Interactive Techniques in Australia and Southeast Asia, pp. 273-280, 2007.

18 "PRWeb: CyberTouch Haptic Glove Used to Show Feasibility of 3D Maintenance Training and Display System," 2012. [Accessed: July 30, 2014]. [Website]. Available: http://www.prweb.com /releases /2012/11/ prweb10178291.htm.

19 "RNI: RNI Demonstrates HitBox Haptics Systems," [Accessed: July 30, 2014]. [Website]. Available: http://www.resrchnet.com/category/rni-news/.

20 "DevinSense Display 300 system: DevinSense Display Solutions," [Accessed: July 30, 2014]. [Website]. Available: http://www.devinsense.com/products/devinsensedisplay-300-2/.

21 A.C. Fyans, G. McAllister, "Creating games with feeling," 2006. Available: http://www.cfyans.com/papers/Creating Games With Feeling.pdf

22 "Unity: Active Stereoscopic 3D for Unity," [Accessed: July 30, 2014]. [Website]. Available: http://u3d.as /content/dembeta-sl/active-stereoscopic-3d-for-unity/2Vt/.

23 A. Van Gelder, "Approximate simulation of elastic membranes by triangulated spring meshes," J. Graphics Tools, vol. 3, pp. 42, 1998.

24 C. S. Mikhael, W. Funnell, M. Bance, "Middle-ear fnite-element modelling with realistic geometry and a priori material-property estimates," Proc. of Canadian Medical and Biological Engineering Society, pp. 126-129, 2004.

25 C. D. Bruyns, S. Senger, "Interactive Cutting of 3D Surface Meshes," Computers \& Graphics, vol. 25, pp. 635-642, 2001.

26 S. J. Hamstra, R. Brydges, R. Hatala, B. Zendejas, D. A. Cook, "Reconsidering fidelity in simulation-based training," Academic Medicine, vol. 89, pp. 387-392, 2014.

27 T. McInerney, D. Terzopoulos, "Deformable models in medical image analysis: a survey," Medical Image Analysis, vol.1, pp. 91-108, 1996.

28 A. C. Hodge, A. Fenster, D. B. Downey, H. M. Ladak, "Prostate boundary segmentation from ultrasound images using $2 \mathrm{~d}$ active shape models: optimisation and extension to 3D," Computer methods and programs in biomedicine, vol. 84, pp. 99-113, 2006.

29 "MPB Technologies Inc.: 7 DOF Haptic Interface," [Accessed: July 30, 2014]. [Website]. Available:

http://mpbtechnologies.ca/mpbt/mpbt_web_2009/_en/7dof/index.html. 


\section{Chapter 3. \\ Face and Content Validity of a Virtual-Reality Simulator for Myringotomy with Tube Placement}

This chapter is a post-print of the following research paper which is available online through the journal's open access portal: C. Huang, H. Cheng, Y. Bureau, S. K. Agrawal, H. M. Ladak, "Face and content validity of a virtual-reality simulator for myringotomy with tube placement," Journal of Otolaryngology-Head and Neck Surgery, DOI: 10.1186/s40463-015-0094-2.

\subsection{Introduction}

Myringotomy with tube insertion is one of the most common procedures in Otolaryngology - Head \& Neck Surgery, and is encountered by residents throughout their training. Despite the fact that it is a ubiquitous procedure, the instruction of junior trainees, who often have little experience in microscopic procedures, is often challenging. Montague et al. [1] have analyzed surgical errors through video analysis of actual procedures and note that the 4 most frequently occurring errors in order from most to least occurring include (1) failure to perform a unidirectional myringotomy, (2) making multiple attempts to place the tube, (3) making multiple attempts to complete the myringotomy, and (4) setting the microscope magnification too high. More serious intraoperative complications can also occur including external auditory canal lacerations, medial displacement of tubes into the middle ear, and vascular injuries [2-4]. Although surgical residents can eventually perform standard cases well, they often struggle with 
narrow canals, retracted tympanic membranes, T-tubes, and procedures performed under local anaesthestic. The goal of simulation is to decrease the learning curve prior to entering the operating, minimize complications in patients, and provide the ability to practice difficult cases.

Several physical models have been described in the literature to provide practice without potential harm to patients [5-9]. Generally, these consist of a tube to mimic the ear canal with a synthetic membrane attached to one end to represent the eardrum. These models do not appear to have gained general acceptance in residency programs, presumably because they are not able to represent anatomical variability easily and the mechanical properties of the materials used do not mimic that of the actual tissues.

Compared with physical models, simulators based on virtual-reality (VR) technologies have the ability to simulate difficult anatomy, model various pathologies, provide automated feedback, and even allow trainees to practice on patient-specific models generated from CT/MRI scans. VR-based simulators have been applied in Otolaryngology, especially for endoscopic sinus surgery [10-14] and for temporal bone drilling [15-18].

In VR simulators, the trainee interacts with realistic 3D digital models of anatomical structures and views them using 3D displays. Simulated tissues can be operated upon using digital representations of actual surgical tools that can be moved in the workspace using devices such as a haptic arm. The sensation of contact force between a digital surgical tool and simulated tissue can be computed and applied to the trainee's hand via the haptic arm. 
The Auditory Biophysics Laboratory at Western University has developed and reported on several aspects of VR-based myringotomy simulation. A blade navigation software system $[19,20]$ and a system for real-time deformation and cutting of the tympanic membrane [21] were implemented on different software platforms as separate training modules. These versions of the simulator were not integrated and they did not include speculum placement, operating microscope controls for positioning/zooming, or tube insertion through the myringotomy.

As recently reported [22], the Western myringotomy simulator has integrated the previous modules into a common software platform. Moreover, new software modules have been added to allow the user to adjust their surgical view through positioning and tilting of the virtual speculum and operative microscope, and to allow insertion of a ventilation tube into the myringotomy created in a deformable tympanic membrane. The goal is to further expand this simulator in the future to allow trainees to raise tympanomeatal flaps and eventually perform tympanoplasty/ossiculoplasty on patientspecific anatomy.

In order for training simulators to be accepted into a residency curriculum, a variety of validation studies need to be conducted starting with face validity and culminating in the demonstration that skills acquired in the VR environment transfer to the OR (operating room) environment. Face validity refers to the degree to which a simulation appears like the real situation [23] and content validity measures whether the simulator would be appropriate or useful in training [24, 25]. Although face validity has previously been established for individual software modules [19-21], validation testing has not been 
performed on the current integrated system, which simulates the entire procedure from microscope positioning to ventilation tube insertion [22].

The objective of this paper is to determine the face and content validity of the new integrated Western myringotomy simulator.

\subsection{Methods}

\subsubsection{Simulator}

An overview of the major features of the simulator is given here; in-depth technical details on the system can be found in a previous publication [22]. The simulator consists of 3 major components: the simulation software, a display system, and a haptic arm as shown in Figure 3.1. The simulation software was developed in the Auditory Biophysics Laboratory at Western University [19-22]. The simulator runs on a Z420 Hewitt-Packard personal computer, equipped with an Intel(R) Xeon E5-1620 processor (Intel Corp., Sanata Clara, CA) and a NVIDIA Quadro 4000 graphics card (NVIDIA Corp., Santa Clara, CA). The system is capable of real-time rendering of the 3D digital models of the ear, surgical tools, and tympanic membrane as shown in Figure 3.2-a. The simulator can import various ear canal and tympanic membrane models, however for the purposes of this study, a normal pediatric ear canal and tympanic membrane was used. The system also incorporates multi-point collision detection to monitor for all interactions between the virtual tools and virtual ear and performs real-time deformation and tissue cutting as required. The software displays the models and all interactions on a silver screen mirror that is part of the DevinSense Display 300 system (DevinSense Display Solutions, Sundbyberg, Sweden). When the screen is viewed using active 3D glasses (Nvidia Corp., 
Santa Clara, CA) provided with the DevinSense system, the 3D digital scene consisting of the virtual ear and tools appears to exist in the space below the silver screen mirror. The display in this region is correctly co-located with the haptic arm (Omni haptic arm, Geomagic, Inc., Morrisville, NC) so movements of the haptic arm appear to occur in the same space as the 3D scene. Using the haptic arm, the user can move the virtual surgical tools. Currently, a single haptic arm is used to control the various instruments, however a second haptic arm could be added to simultaneously manipulate multiple instruments (e.g. speculum and myringotomy blade).

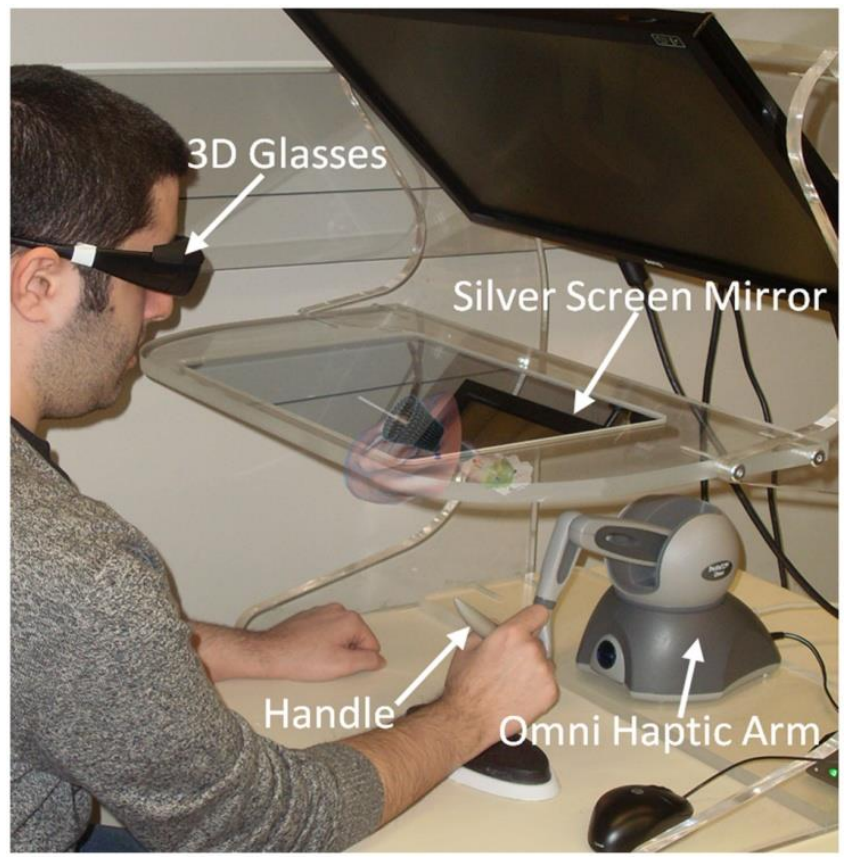

Figure 3.1 Simulator set up. A user is shown using the Western myringotomy simulator. By moving the handle of the haptic arm, the user controls the movement of a virtual myringotomy blade and forceps. The virtual ear and tools floating under the silver screen mirror are an artistic rendering of what the user would see through the 3D glasses.

The haptic arm can be used to position and rotate the virtual speculum, position and tilt the microscope, and adjust magnification to obtain different views of the operative site as 
shown in Figure 3.2-b. The user can then create a myringotomy as shown in Figure 3.2-c using a virtual myringotomy blade; the position and orientation of the blade are controlled by moving the handle of the haptic arm. A tube may be inserted using virtual forceps, which is also controlled by the user using the haptic device [Figure 3.2-d]. The opening and closing of the forceps can be toggled using a button on the haptic arm. During tube insertion, the eardrum deforms and the incision splays as the tube enters the myringotomy. The tube may also be repositioned with various instruments until it is in its final position [Figure 3.2-e]. 


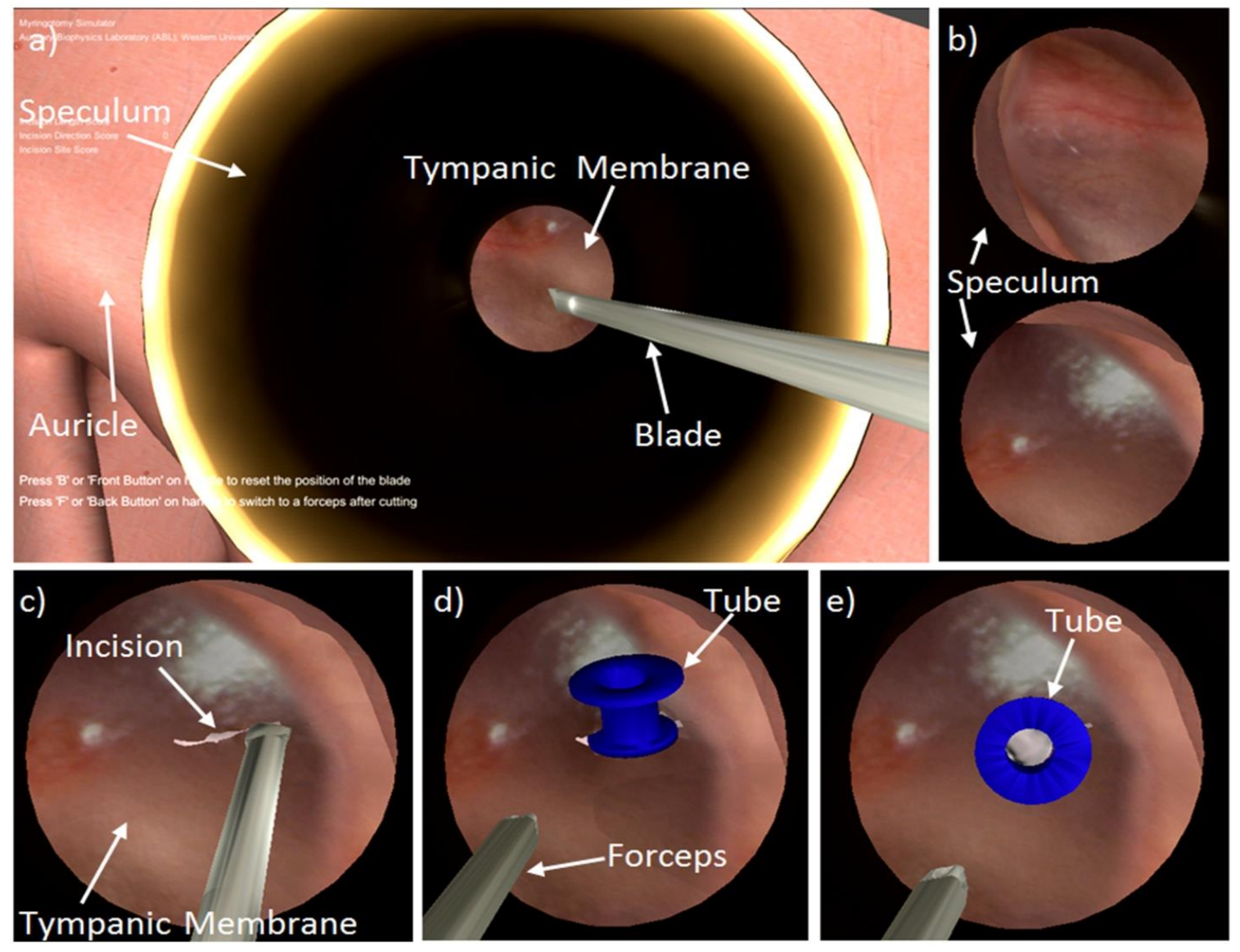

Figure 3.2 Simulator scene shown in 2D. The actual scene would be viewed by the user in stereoscopic 3D. a) View of the speculum and myringotomy blade. b) Magnified views of the tympanic membrane through the speculum (represented by the black circle). The view changes depending on the (i) magnification and (ii) position and tilt of the speculum and microscope. c) Myringotomy d) Tube insertion and splaying of the incision. e) Tube in final position with middle ear visible through the lumen of the tube.

\subsubsection{Participants}

Research ethics board approval was obtained from Western University (\#105239; Appendix A) and participants were contacted via telephone or electronic mail. All participants were recruited from the Department of Otolaryngology - Head \& Neck Surgery, Western University. A total of 12 subjects agreed to participate, which included seven junior Otolaryngology residents (postgraduate years 1 to 3 ) and five senior 
Otolaryngologists who routinely performed ventilation tube insertions in their practice. These groups were chosen to reflect the target group of the simulator (junior residents) as well as experts in the field (Otolaryngologists). The participants did not have any previous exposure to myringotomy simulation.

\subsubsection{Protocol}

All participants were initially given an orientation session which consisted of: 1) an information sheet outlining the software features of the simulator, 2) a demonstration video of how to perform a myringotomy and tube insertion using the simulator controls, and 3) a live demonstration of the simulator and haptic arm. The same graduate student and surgical resident performed the orientation session for each participant, and a standardized script was used to ensure consistency. The participants were specifically asked to perform the tasks listed in Table 3.1 so that they could comment on all the various aspects of the simulator. Finally, the participants were given an unlimited period of time to use the simulator until they felt comfortable completing the face and content validity questionnaires.

Table 3.1 Tasks involved in the face validity study.

\begin{tabular}{l|l}
\hline Tasks & Description \\
\hline Speculum & Rotate and tilt the speculum to obtain view of tympanic \\
Microscope & Translate and rotate the microscope to obtain a proper view \\
Blade navigation & Navigate surgical blade through the external auditory canal \\
Myringotomy & Make an incision in the tympanic membrane \\
Ventilation tube & Insert ventilation tube into the myringotomy using forceps \\
\hline
\end{tabular}




\subsubsection{Questionnaire}

Previously, we had tested individual software modules focusing on blade navigation [19], haptics [20] and tympanic membrane deformation and cutting [21]. Since this new simulator [22] refined each of these components, including the graphical representations of the ear and virtual tools, and included new features such as microscope handling, speculum positioning and tube insertion, the Myringotomy Surgery Simulation Scale (MS3) used in previous publications [20,21] was modified to include these features. The questionnaire was divided into three sections (A, B, and $\mathrm{C}$ ) with a total of 20 questions. Section A included 14 questions focusing on face validity as listed in Table 3.2. The appearance and realism of the surgical instruments; anatomy of the auricle, ear canal and eardrum; movement of surgical instruments; deformation and cutting of the eardrum; tube insertion and 3D microscopic view of the scene were assessed.

Table 3.2 Questions in Section A for face validity

\begin{tabular}{c|l}
\hline No. & $\begin{array}{l}\text { Question : Rate whether the following aspects of the simulator } \\
\text { are realistic }\end{array}$ \\
\hline 1 & $\begin{array}{l}\text { Visual appearance of the auricle and ear canal } \\
2\end{array}$ \\
3 & Visual appearance of the speculum \\
4 & Movement of the speculum \\
5 & Movement of the microscope/camera \\
6 & Zoom of the microscope/camera \\
7 & Visual appearance of the eardrum \\
8 & Movement of the eardrum when physically contacted \\
9 & Visual appearance of the myringotomy blade \\
10 & Visual appearance and splay of the myringotomy \\
11 & Movement and stability of the myringotomy blade and forceps \\
12 & Visual representation of the tube \\
13 & Movement of the tube within the myringotomy \\
14 & Three-dimensional microscopic view of the scene based on light rendering, \\
& shadows, and 3D goggles \\
\hline
\end{tabular}


Section B included six questions focusing on content validity as listed in Table 3.3. These questions were used to determine training potential on specific surgical tasks.

Table 3.3 Questions in Section B for training potential

\begin{tabular}{c|l}
\hline No. & $\begin{array}{l}\text { Question: Do you feel that the simulator would be useful in } \\
\text { teaching Otolaryngology trainees the following skills }\end{array}$ \\
\hline 15 & Speculum placement \\
16 & Microscope positioning \\
17 & Tool navigation \\
18 & Ear canal and eardrum anatomy \\
19 & Myringotomy creation \\
20 & Tube insertion \\
\hline
\end{tabular}

In Sections A and B, study participants were asked to answer each question using a 7point Likert scale, an equal appearing interval measurement. The scale had values of 1"Strongly Disagree", 2-“Mostly Disagree”, 3-“Disagree", 4-“Neither Agree/Disagree”, 5 -“Agree", 6-"Mostly Agree" and 7-“Strongly Agree".

In Section C, a free-form comment area was provided for each participant to provide feedback to elaborate on previous questions and to address issues not covered in Sections A and B.

\subsubsection{Statistical analysis}

The responses were initially divided by group (junior resident or practising Otolaryngologist), and the median, quartiles, minimum, and maximum response values were computed for each question. The sample size was maximized to include all eligible participants at a single academic institution. For each question, the Mann-Whitney U-test was used to test the significance of the differences in responses between the two groups. A frequency distribution histogram was plotted to investigate the number of favourable 
responses $($ score $\geq 5)$, neutral responses $($ score $=4)$, and negative responses $($ score $\leq 3)$ to each question. All data were computed and analysed using the SPSS statistical software (SPSS Inc, Chicago, IL). The significance was set at $\mathrm{p}<.05$ and the Holm-Bonferroni method was used to correct for multiple comparisons.

\subsection{Results}

\subsubsection{Demographics}

The first group was comprised of seven junior Otolaryngology residents in postgraduate years 1 to 3 . They were all familiar with the operating microscope and the procedure, however they were in the active phase of learning with each resident having performed fewer than 20 myringotomy and tube insertions in training. The second group had five fellowship trained Otolaryngologists who routinely performed myringotomy and tube insertions in their practice. Each member of this group had performed at least 200 procedures since completing their fellowship.

\subsubsection{Comparison of groups}

The mean response and confidence interval for each question in Section A (face validity) and Section B (content validity) are summarized in Figure 3.3. Application of the MannWhitney U-test indicates no statistically significant differences between residents and senior Otolaryngologists. However, the largest differences between the groups were seen in Question $13(\mathrm{U}=5.5, \mathrm{p}=0.043)$ and Question $20(\mathrm{U}=7, \mathrm{p}=0.097)$, which related to the movement of the tube within the myringotomy. 


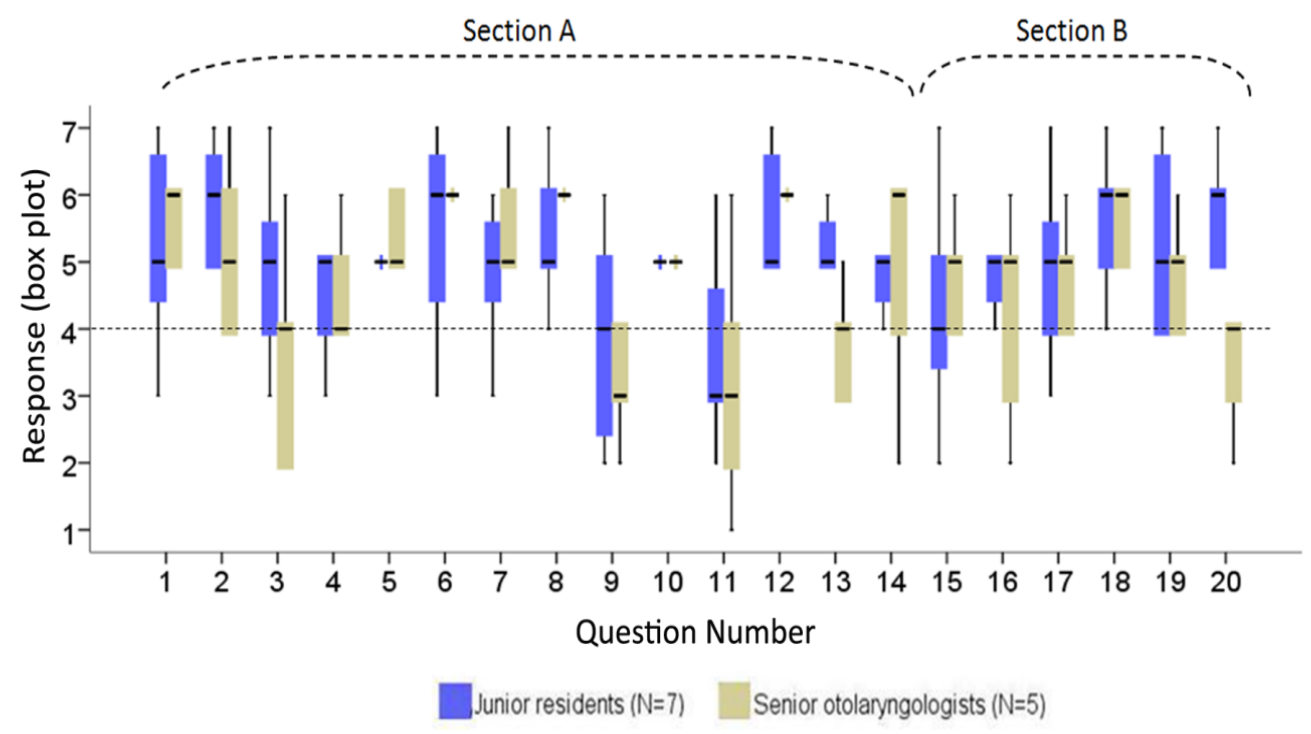

Figure 3.3 Box plot of the Likert item responses for the two groups of participants. Face validity was assessed in Questions 1-14, and content validity was assessed in Questions 15-20. A response of 4 is neutral, and higher values are more favourable than lower values.

\subsubsection{Face and Content Validity}

Given that mean responses were not different at the $\mathrm{p}=.05$ level, the results for the two groups were pooled when analyzing face and content validity. The responses to the questionnaires were categorized as positive (score $\geq 5$ ), neutral $($ score $=4)$ or negative (score $\leq 3)$.

\section{Face validity}

The realism of the simulator was investigated through the 14 questions in Section A of the questionnaire. As can be seen in Figure 3.4, the number of positive responses exceeds the number of neutral and negative responses except in the case of Questions 9 and 11. Question 9 focuses on the realism of the visual appearance and splay of the myringotomy, 
whereas Question 11 focuses on the realism of the movement and stability of the myringotomy blade and forceps. Overall, when the 14 questions over 12 participants (168 total responses) were considered, there were $116(69.0 \%)$ positive responses, $21(12.5 \%)$ neutral responses, and $31(18.5 \%)$ negative responses.

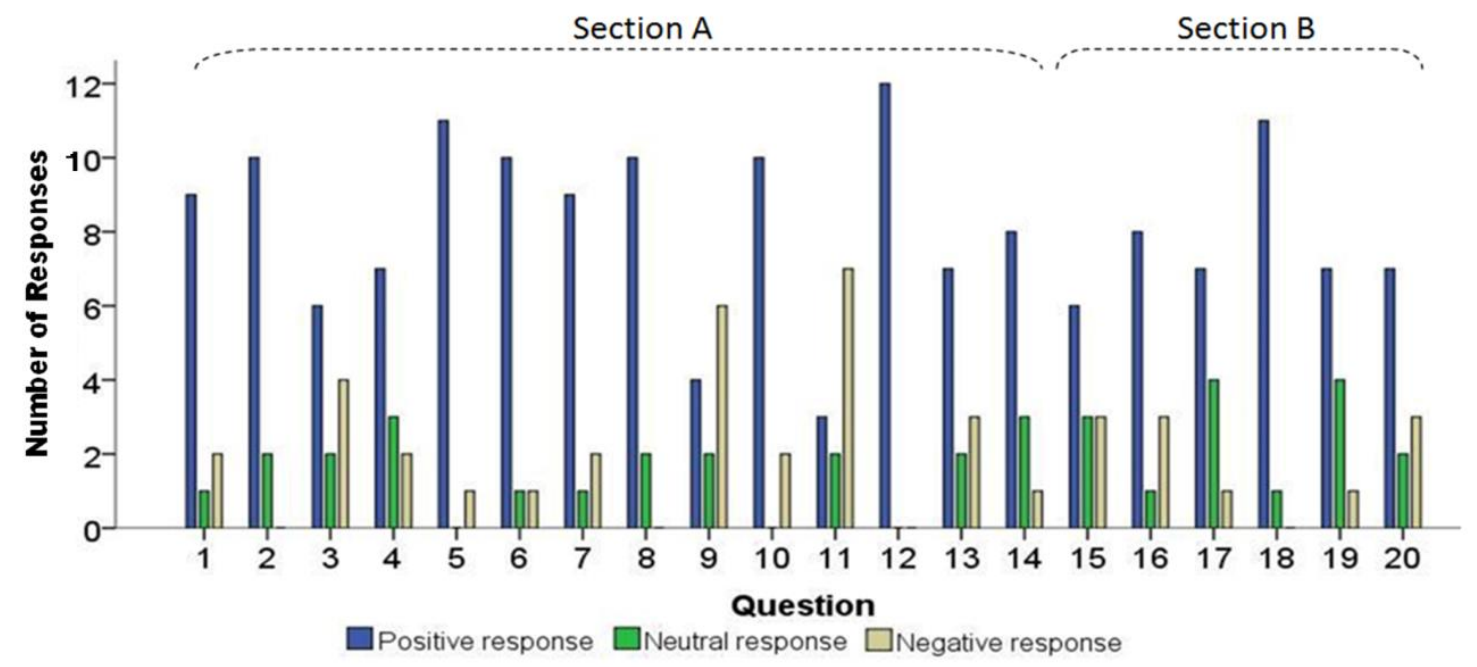

Figure 3.4 Total number of positive, neutral and negative responses to each question, pooling responses of junior residents and of senior Otolaryngologists. The blue bar indicates the number of positive responses (score $\geq 5$ ), the green bar is the number of neutral responses (score $=4$ ), and the beige bar indicates the number of negative responses (score $\leq 3$ ).

\section{Content validity}

The training potential of the simulator was tested through 6 questions in Section B of the questionnaire. As shown in Figure 3.4, the number of positive responses was greater than the number of negative responses for each question in this section. Among the total 72 
responses (6 questions x 12 participants), 46 (63.9\%) were positive, 15 (20.8\%) were neutral, and $11(15.3 \%)$ were negative.

\subsection{Discussion}

The MS3 scale used in this study had to be developed at our institution as no other validated measure was available to assess a virtual-reality myringotomy simulator. This questionnaire has not been externally validated by other centres, however content validity was assessed by a group of experts during the development of the questionnaire. In addition, previous publications [20,21] did demonstrate reliability of the MS3 with a strong correlation across raters. The MS3 was also correlated against a visual analogue scale measuring the same construct, thus providing us with a measure of concurrent validity [21].

The lack of statistically significant differences in mean responses between residents and senior Otolaryngologists to Questions 1 to 20 at the $\mathrm{p}=.05$ level suggests that even with limited exposure to the actual procedure of myringotomy with tube insertion, junior residents had similar assessments of the realism and utility of the simulator as those experienced in the OR.

The only differences between the groups approaching significance were in Questions 13 and 20, which pertained to the movement of the tube within the myringotomy. Senior Otolaryngologists perceived the simulated tube movement to be less realistic than did residents. Similarly, Question 9 in the pooled responses dealt with the splay of the myringotomy, and this had a higher number of negative responses overall. From the written comments in Section $\mathrm{C}$ of the questionnaire, it appears that splaying (i.e., 
spreading) of the virtual eardrum when it is contacted by the virtual blade is realistic, and this was also the case in our previous report [21]; however, splaying is less realistic during tube insertion when the virtual tube contacts the eardrum and causes it to spread.

This difference could be explained by a design decisions made during the development of the tube insertion module. First, although the tympanic membrane has real-time deformation, the physics of the interaction between the edges of the myringotomy and a ventilation tube is quite complex. In order to detect contact with the tube, the tympanic membrane is represented as a discrete collection of spatially distributed points as shown in Figure 3.5. Collision detection is performed at each of these discrete contact points. When the spatial density of points is high (i.e. the points are close together) the location of contact can be calculated with more precision than when the spatial density is lower. Unfortunately, multi-point collision detection is computationally intensive, therefore the rendering speed decreases rapidly as the spatial density and precision is increased. The particular choice of density in the simulator was chosen to permit animations to occur at a realistic pace on an inexpensive personal computer, however this negatively affected the precision of the tympanic membrane splay in response to the tube. 


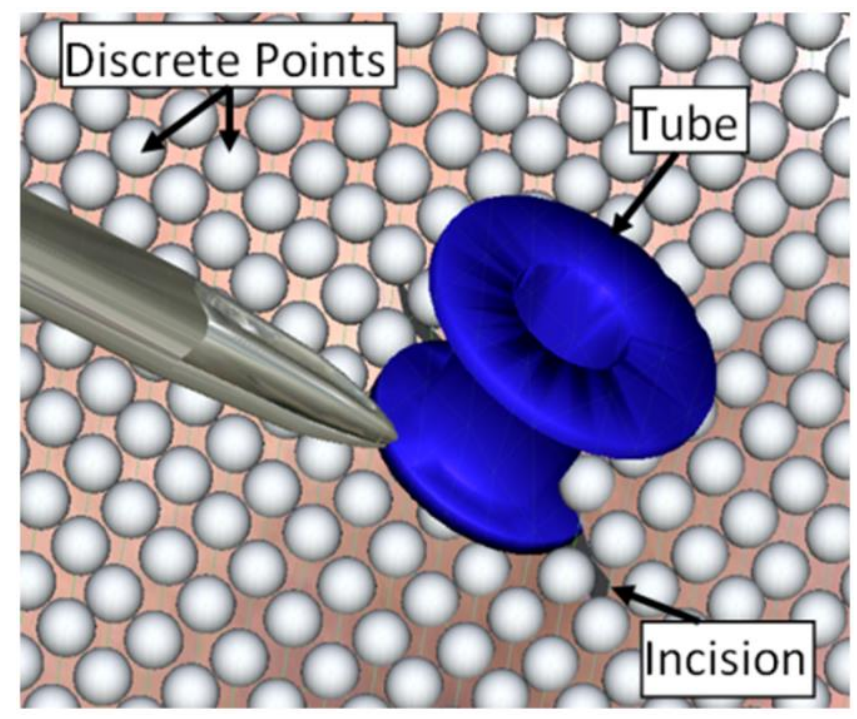

Figure 3.5 Representation of the virtual tympanic membrane by a collection of discrete points. The points define the geometry of the tympanic membrane and act as contact detectors with the virtual instruments (myringotomy blade, forceps, and ventilation tube).

Second, the physics of tympanic membrane 'tearing' with large forces and displacements during tube insertion are difficult to model in real-time. To overcome this, preprogrammed animations were used based on the length of incision, the trajectory of the tube, and the contact between the flange of the tube and the myringotomy. Although this significantly reduced computation time, Question 13 revealed that this lack of realism was noted by the experts and not the residents. This could be explained by the fact that senior surgeons would have had much more experience knowing how the ventilation tube should slide into the incision, therefore they were able to notice the subtle differences more than the junior trainees still learning the procedure. On average, Otolaryngologists' rankings fell between "Disagree" to "Neither Agree/Disagree", suggesting that slight improvements to the tube insertion simulation could make this aspect more acceptable. 
Question 11 was the only other question with a higher proportion of negative responses, and this pertained to the movement and stability of the blade and forceps. Section C clarified this finding as concerns were raised about the limited range of motion of the haptic device and that the friction of the device affected the movements of the virtual blade and forceps. The haptic arm used in this study is a low-cost device that is suitable for design of a prototypical simulator. The device can easily be swapped for a higher fidelity device with greater range of motion and substantially reduced friction (e.g., Geomagic Phantom Premium device from Geomagic, Inc., Morrisville, NC), albeit at greater financial cost. Utilizing the higher fidelity device may result in acceptable range of motion and unnoticeable friction. A second concern with the device was the feel of the handle of the haptic arm when it was used to control the blade and forceps (Figure 3.1). As the handle is thick, it feels unnatural compared to holding an actual surgical tool. We have implemented approaches described in the literature to replace the haptic arm handle with actual surgical tools to improve the feel and realism of the simulation [26]. The goal in this hybrid simulator would be have one haptic arm attached to a myringotomy blade or forceps, and have the second haptic arm attached to a real speculum to maximize realism.

Face and content validity are only initial steps in validation, and they do not ensure that a simulator will be useful in training residents [24, 25]. Future development on the Western myringotomy simulator will address concerns raised in this study. Refinement and optimization of the tube insertion and tympanic membrane splay may help to increase the realism of the simulator, but it is unclear if increased fidelity will actually result in additional skills transference [27]. In order to determine the construct validity of 
the simulator, automated metrics including time, length and direction of incision, collisions, magnification, etc. have been incorporated into the simulator. A separate study will examine if these metrics are capable of distinguishing experts from residents, and a skills transference study will be needed to determine if the simulator can result in better operating room performance. A multi-centred study will be considered at that time to maximize sample size and feedback from different centres.

The authors hope that by using standardized libraries while programming the simulator, and the ability of the simulator to run on low-cost hardware, will allow easy adoption by Otolaryngology training programs and allow other groups to make modifications as needed.

\subsection{Conclusion}

The Western myringotomy simulator has a number of new features including microscope handling, speculum positioning and ventilation tube insertion. The simulator has good face and content validity, except with respect to splaying of the myringotomy during tube insertion and with respect to haptic arm. These issues are currently being addressed with further refinements and adaptations. Automated metrics have been developed and they will be used to assess for construct validity of the simulator. Although the entire myringotomy and ventilation tube insertion can now be simulated, a skills transference study is needed to establish training efficacy and clinical impact. 


\subsection{References}

1 M. Montague, M. S. W. Lee, S. S. M. Hussain, "Human error identification: an analysis of myringotomy and ventilation tube insertion," Archives of Otolaryngology-Head and Neck Surgery, vol. 130, no. 10, pp. 1153-1157, 2004.

2 B. N. Brodish, A. L. Woolley, "Major vascular injuries in children undergoing myringotomy for tube placement," American Journal of Otolaryngology, vol. 20, pp. 46-50, 1999.

3 M. Kumar, A. M. Khan, S. Davis, "Medial displacement of grommets: an unwanted sequel of grommet insertion," The Journal of laryngology and otology, vol. 114, pp. 448-449, 2000.

4 J. C. Groblewski, E. H. Harley, "Medial migration of tympanostomy tubes: an overlooked complication," International Journal of Pediatric Otorhinolaryngology, vol. 70, pp. 1707-1714, 2006.

5 T. Walker, S. Duvvi, and B. N. Kumar, "The Wigan Grommet Trainer," Clinical Otolaryngology, vol. 31, no. 4, pp. 349-350, 2006.

6 M. Duijvestein, and J. Borgstein, "The Bradford Grommet Trainer," Clinical Otolaryngology, vol. 31, no. 2, pp. 163-163, 2006.

7 A. Leong, S. Kundu, P. Martinez-Devesa, C. Aldren, "Artificial ear: a training tool for grommet insertion and manual dexterity," ORL : Journal for Oto - Rhino Laryngology and Its Related Specialties, vol. 68, no. 2, pp. 115-117, 2006.

8 P. Hong, A. N. Webb, G. Corsten, J. Balderston, R. Haworth, K. Ritchie, E. Massoud. "An anatomically sound surgical simulation model for myringotomy and tympanostomy tube insertion," International Journal of Pediatric Otorhinolaryngology, vol 78, pp. 522-529, 2008.

9 P. G. Volsky, B. B. Hughley, S. M. Peirce, B. W. Kesser, "Construct validity of a simulator for myringotomy with ventilation tube insertion, "Otolaryngology-Head and Neck Surgery, vol. 141, pp. 603-608, 2009.

10 S. Weghorst, C. Airola, P. Oppenheimer, C. V. Edmond, T. Patience, D. Heskamp, J. Miller, "Validation of the Madigan ESS Simulator," Studies in Health Technology and Informatics, vol. 50, pp. 399-405, 1998.

11 S. M. Anil, Y. Kato, M. Hayakawa, K. Yoshida, S. Nagahisha, T. Kanno, "Virtual 3dimensional preoperative planning with the Dextroscope for excision of a 4 th ventricular ependymoma," Minimally Invasive Neurosurgery, vol. 50, pp. 65-70, 2007.

12 M. Audette, H. Delingette, A. Fuchs, O. Astley, K. Chinzei, "A topologically faithful, tissue-guided, spatially varying meshing strategy for computing patient-specific head models for endoscopic pituitary surgery simulation," Studies in Health Technology and Informatics, vol. 119, pp. 22-27, 2006.

13 B. Tolsdorff, A. Pommert, K. H. Höhne, A. Petersik, B. Pflesser, U. Tiede, R. Leuwer, "Virtual reality: a new paranasal sinus surgery simulator," Laryngoscope, vol. 120, pp. 420-426, 2010.

14 R. Varshney, S. Frenkiel, L. H. P. Nguyen, M. Young, R. Del Maestro, A. Zeitouni, E. Saad, W. R. J. Funnell, M. A. Tewfik, National Research Council 
Canada, "The McGill simulator for endoscopic sinus surgery (MSESS): a validation study," Journal of Otolaryngology-Head and Neck Surgery, vol. 43, pp. 40, 2014.

15 G. J. Wiet, D. Stredney, T. Kerwin, B. Hittle, S. A. Fernandez, M. Abdel-Rasoul, D. B. Welling, "Virtual temporal bone dissection system: development and testing," The Laryngoscope, vol. 122(Suppl. 1), pp. S1-S12, 2012.

16 D. Morris, C. Sewell, F. Barbagli, K. Salisbury, N. H. Blevins, S. Girod, "Visuohaptic simulation of bone surgery for training and evaluation," IEEE Computer Graphics and Applications, vol. 26, pp. 48-57, 2006.

17 C. Sewell, D. Morris, N. H. Blevins, S. Dutta, S. Agrawal, F. Barbagli, K. Salisbury, "Providing metrics and performance feedback in a surgical simulator," Computer Aided Surgery, vol. 13, pp. 63-81, 2008.

18 A. Arora, S. Khemani, N. Tolley, A. Singh, J. Budge, D. A. Varela, H. W. Francis, A. Darzi, N. I. Bhatti, "Face and content validation of a virtual reality temporal bone simulator," Otolaryngology-Head and Neck Surgery, vol. 146, pp. 497-503, 2012.

19 B. Wheeler, P. C. Doyle, S. Chandarana, S. Agrawal, M. Husein, H. M. Ladak, "Interactive computer-based simulator for training in blade navigation and targeting in myringotomy," Computer Methods Programs in Biomedicine, vol. 98, no. 2, pp. 130-139, 2010.

20 L. J. Sowerby, G. Rehal, M. Husein, P. C. Doyle, S. Agrawal, H. M. Ladak, "Development and face validity testing of a three-dimensional myringotomy simulator with haptic feedback," Journal of Otolaryngology: Head and Neck Surgery, vol. 39, no. 2, pp. 122-129, 2010.

21 A. K. Ho, H. Alsaffar, P. C. Doyle, H. M. Ladak, S. K. Agrawal, "Virtual reality myringotomy simulation with real-time deformation: development and validity testing," The Laryngoscope, vol. 122, no. 8, pp. 1844-1851, 2012.

22 C. Huang, S. K. Agrawal, H. M. Ladak, "Virtual-reality simulator for training in myringotomy with tube placement," Proceedings of the 37th Canadian Medical and Biological Engineering Conference, May 2014.

23 F. J. Carter, M. P. Schijven, R. Aggarwal, T. Grantcharov, N. K. Francis, G. B. Hanna, J. J. Jakimowicz, "Consensus guidelines for validation of virtual reality surgical simulators," Surgical Endoscopy, vol. 19, no. 12, pp. 1523-1532, 2005.

24 A. G. Gallagher, E. M. Ritter, R. M. Satava RM, "Fundamental principles of validation, and reliability rigorous science for the assessment of surgical education and training," Surgical Endoscopy, vol. 17, no. 10, pp. 1525-1529, 2003.

25 B. M. A. Schout, A. J. M. Hendrikx, F. Scheele, B. L. H. Bemelmans, A. J. J. A. Scherpbier, "Validation and implementation of surgical simulators: a critical review of present, past, and future," Surgical Endoscopy, vol. 24, no. 3, pp. 536-546, 2010.

26 T. R. Coles TR, N. W. John, G. Sofia, D. A. Gould, D. G. Caldwell, "Modification of commercial force feedback hardware for needle insertion simulation," MMVR18 Medicine Meets Virtual Reality 2011 Poster, February 2011.

27 S. J. Hamstra, R. Brydges, R. Hatala, B. Zendejas, D. A. Cook, "Reconsidering fidelity in simulation-based training," Academic Medicine, vol. 89, pp. 387-392, 2014. 


\section{Chapter 4.}

\section{Automated Quantitative Metrics in a Virtual- reality Myringotomy and Tube Simulator: Development and Construct Validity}

This article is a preprint of the following article: C. Huang, H. Cheng, Y. Bureau, H. M. Ladak, S. K. Agrawal, “Automated quantitative metrics in a virtual-reality myringotomy and tube simulator: Development and construct validity, The Laryngoscope, Submitted in December, 2015.

\subsection{Introduction}

Although myringotomy with tube insertion is one of the most common procedures in Otolaryngology - Head \& Neck Surgery, junior trainees can often find the procedure difficult because of retracted tympanic membranes, narrow external auditory canals, and when performed in a clinic setting. Montegue et al.[1] described the most common errors during myringotomy, however more serious complications including vascular injuries have been reported [2-4].

To date, physical models have primarily been used in order to allow trainees to practice prior to entering the operating room [5-12]. These models generally consist of a tube with a synthetic membrane to model the external auditory canal and eardrum. These models have the advantage of being relatively inexpensive, however only one has established construct validity [9]. Mahalingam et al. [13] recently reviewed the literature and 
independently assessed ventilation tube insertion simulators; unfortunately, none of the five simulators assessed achieved face or global content validity.

Virtual-reality (VR) simulators have been increasingly developed and adopted with the advances in technology. In the field of laparoscopic surgery, many studies have been shown that these simulators result in skills transference in the operating room [14]. In Otolaryngology, a number of VR simulators have been described for mastoidectomy and endoscopic sinus surgery [15-23], however validation of skills transference has been lacking [24]. These simulators are technically challenging to develop, but they have the advantage of being able to model various pathologies, simulate difficult cases, and allow trainees to practice on patient-specific models.

One of the main challenges in simulator adoption by training programs has been the continued need for an instructor to be present to provide feedback [22]. As VR simulators can capture and analyze the learning environment, automated metrics and feedback can be incorporated allowing surgical residents to practice and train independently. Automated performance metrics have been described in the Stanford Surgical Simulator [22], Voxel-Man Temposurg Simulator [25], Mediseus Surgical Drilling Simulator [26], Endoscopic Sinus Surgery Simulator (ES3) [27], and McGill Simulator for Endoscopic Sinus Surgery [28].

The Auditory Biophysics Laboratory at Western University has developed a VR myringotomy and tube simulator capable of running on inexpensive hardware. Various aspects have been incorporated including blade navigation [29], real-time deformation of the tympanic membrane [30], operating microscope manipulation, and tube insertion [31]. 
Face and content validity was also established for the integrated simulator [32], however there have been no metrics or ability to conduct construct validity testing to date.

The primary objective of this study was to design and implement a set of automated performance metrics into the Western myringotomy simulator based upon previously published metrics [22, 25-28], described errors in myringotomy [1], and objective structured assessments [33]. The second objective was to perform a discriminant validity study of these metrics to establish construct validity.

\subsection{Materials and methods}

\subsubsection{Simulator}

The technical details of the Western myringotomy simulator have been previously described [30-32]. Active 3D glasses (Nvidia Corp., Santa Clara, CA) are mounted on a stand to obtain a stereoscopic view of the simulation. The user controls the instruments with a haptic arm (Omni haptic arm, Geomagic, Inc., Morrisville, NC), and this also allows them to feel force feedback whenever contact is made with structures in 3D space. The simulator in this study was run on a Z420 Hewitt-Packard personal computer, equipped with a NVIDIA Quadro 4000 graphics card (NVIDIA Corp., Santa Clara, CA) and an Intel(R) Xeon E5-1620 processor (Intel Corp., Santa Clara, CA). With current graphical processing units providing much better performance at a lower cost, fairly inexpensive hardware can be used to run the simulator with real-time soft tissue deformation and multi-point collision detection. 


\subsubsection{Development and implementation of automated metrics}

The overall simulation and kinematics data of the participants was recorded by the simulator. Custom metrics were then programmed and used to analyze the captured data. The five primary performance metrics were time to completion, surgical errors, incision angle, incision length, and the magnification of the microscope.

The simulator measured the time taken to adjust the microscope, adjust the speculum, perform the myringotomy, and insert the ventilation tube. The total time to completion was then calculated from the activation of the microscope until the tube was placed into the myringotomy.

Surgical errors were based upon those described by Montegue et al. [1]. The location of the virtual instruments was tracked, and any contact with the external auditory canal or middle ear structures constituted an error. Participants were clearly instructed to perform a myringotomy in the inferior half of the tympanic membrane, therefore incisions crossing into the superior tympanic membrane were considered an error. Finally, multiple attempts at completing the myringotomy or tube insertion, along with any associated trauma, were also collected.

Participants were asked to perform a radial incision in the tympanic membrane, and any deviation from the radial line was measured by the value $\theta$ (Figure 4.1). The incision angle was measured as the angle between the myringotomy and the radius as drawn through the umbo (point $\mathrm{O}$ ) and the centre of the incision (point $\mathrm{A}$ ). The angle $\theta$ ranged from 0 to 90 degrees, with 0 degrees representing a perfectly radial incision, and 90 degrees representing a circumferential incision. 


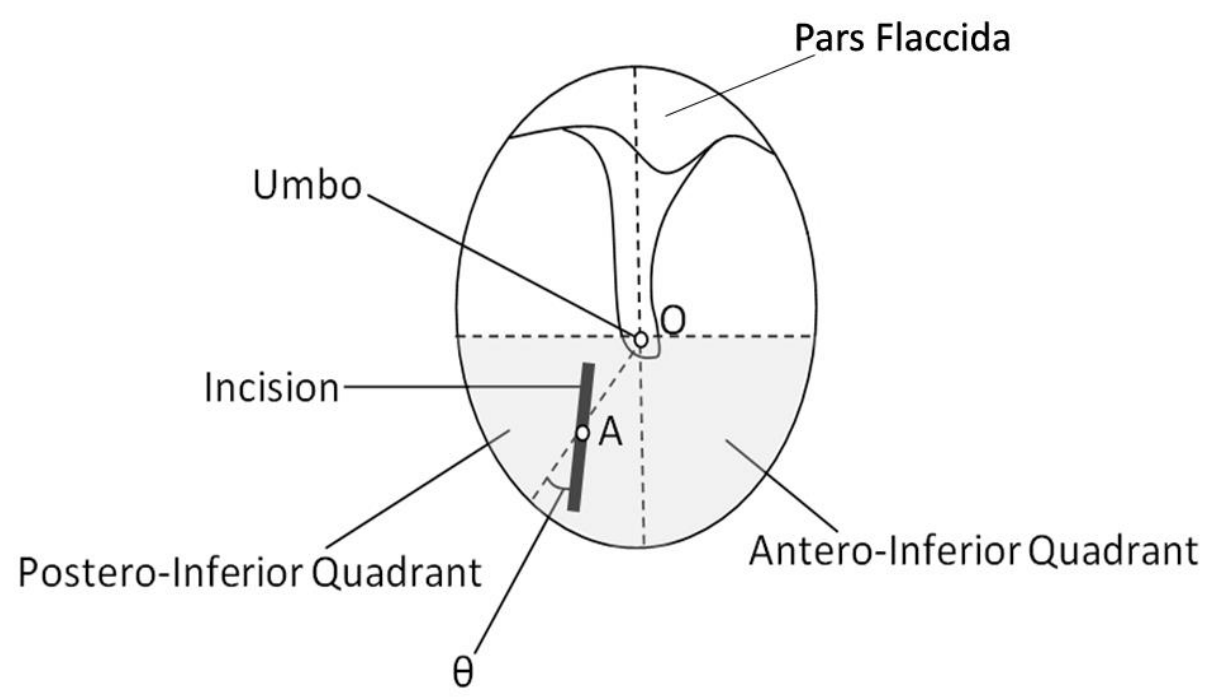

Figure 4.1 Illustration of incision angle $\theta$. The shaded area represents the inferior half of the tympanic membrane. The umbo is marked as point ' $O$ ', and the radial line between the umbo and annulus is shown with a dotted line. The intersection between the centre of the incision and the radial line is marked as point ' $A$ '. The value $\theta$ is the angle between the incision and the radial line along points $O$ and $A$.

Incision length was calculated as the actual length of the myringotomy from the start point to the end point. The outer diameter of the modeled tube was $1.5 \mathrm{~mm}$.

The magnification of the microscope was calculated by the distance D as illustrated in the Figure 4.2. The simulator captured the microscopic view as the myringotomy was made, and the value of $\mathrm{D}$ was measured as the number of pixels visible at the medial end of the speculum. The value of $\mathrm{D}$ increased with higher microscope magnification and was used as a marker of the participants' field of view during the procedure. In addition, the simulator captured whether the umbo and/or annulus were visible as landmarks during the myringotomy. 


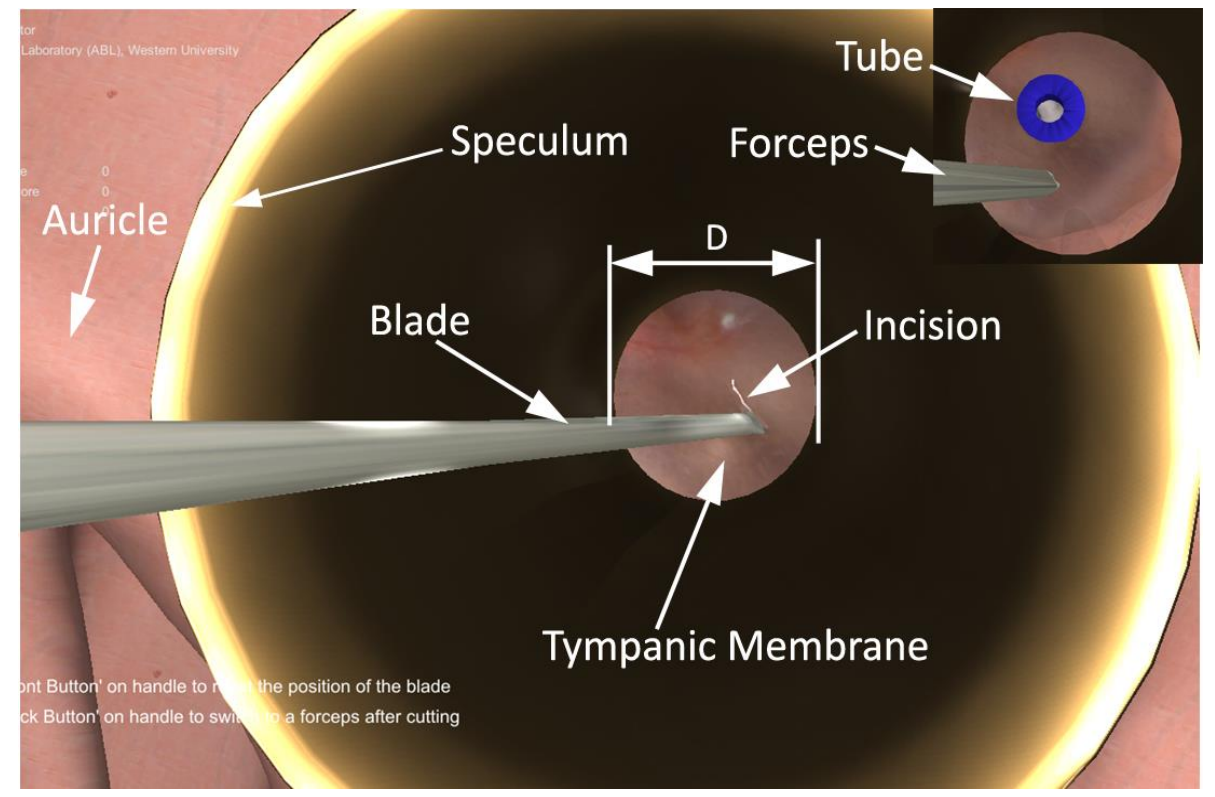

Figure 4.2 A two-dimensional view from the simulator is shown. The value of $D$ is calculated as the number of pixels visible at the medial end of the speculum during the myringotomy. This value increases with higher magnification of the microscope. The inset shows a ventilation tube being inserted into a myringotomy with forceps.

\subsubsection{Subjects}

Research ethics board approval was obtained from Western University, and all participants were recruited from the Department of Otolaryngology - Head \& Neck Surgery. Eleven participants $(\mathrm{N}=11)$ volunteered for the study including seven junior residents (postgraduate years 1 to 3 ) and four senior Otolaryngologists who routinely performed myringotomy and tube insertion. The participants had not had any prior exposure to myringotomy simulation.

\subsubsection{Protocol}

Each participant was given a standardized orientation session outlining myringotomy, tube insertion, and simulator controls. To ensure consistency, a script was used by the 
same graduate student and surgical resident during each session. The participants were then given an unlimited amount of time to practice on a special version of the simulator which had the myringotomy and ventilation tube functions removed. This ensured that all users were comfortable with the $3 \mathrm{D}$ glasses, haptic arm, and adjustment of the microscope/speculum.

Each participant was then asked to 1) obtain the optimum microscopic view, 2) create a radial myringotomy anywhere in the inferior half of the tympanic membrane, and 3) insert a ventilation tube into the myringotomy. This task was outlined to each participant using verbal instructions, diagrams, and an information sheet. Members of both groups then performed 10 trials of myringotomy and tube insertion, and all data was automatically recorded and analyzed by the simulator.

\subsubsection{Statistical analysis}

The performance metrics were analyzed for each group and the mean, standard error of the mean (SEM), and 95\% confidence internals (CI) were calculated for the entire testing session and for each individual trial. The sample size was maximized to include eligible participants at Western University. The Mann-Whitney U-test was used to test differences between the groups, and the Bonferroni method was used to correct for multiple comparisons. The significance level was set at $\mathrm{p}<0.05$. All data were analyzed using SPSS software for Windows (SPSS Inc., Chicago, IL). 


\subsection{Results}

\subsubsection{Demographics}

The junior resident group consisted of seven Otolaryngology residents in postgraduate years 1 to 3 . All residents were familiar with the operating microscope, but had performed fewer than 20 myringotomy and tube insertions in training. The senior otolaryngologists were all fellowship trained and had performed at least 200 myringotomy and tube insertions since entering independent practice.

\subsubsection{Automated Performance Metrics}

A summary of the performance metrics for junior residents and senior otolaryngologists is given in Table 4.1. The individual learning curves over 10 trials are shown in Figures 3 to 7.

Table 4.1 Automated Performance Metrics Over 10 Trials

\begin{tabular}{|l|r|r|r|}
\hline \multicolumn{1}{|c|}{ Performance Metric } & \multicolumn{1}{c|}{$\begin{array}{c}\text { Junior Residents } \\
\text { Mean }(95 \% \mathrm{Cl})\end{array}$} & $\begin{array}{c}\text { Senior Otolaryngologists } \\
\text { Mean }(95 \% \mathrm{Cl})\end{array}$ & Significance \\
\hline Time to completion (seconds) & $68.2(60.5-75.9)$ & $41.5(36.4-46.6)$ & $\mathrm{p}<0.001$ \\
\hline Surgical errors per trial & $0.93(0.71-1.15)$ & $0.33(0.16-0.49)$ & $\mathrm{p}<0.001$ \\
\hline Incision angle $\theta$ (degrees) & $27.1(24.4-29.9)$ & $20.2(17.7-22.7)$ & $\mathrm{P}<0.002$ \\
\hline Incision length $(\mathrm{mm})$ & $1.7(1.6-1.8)$ & $2.1(1.9-2.3)$ & $\mathrm{p}<0.002$ \\
\hline $\begin{array}{l}\text { Microscope magnification } \\
\text { (pixels) }\end{array}$ & $415(397-433)$ & $319(305-334)$ & $\mathrm{p}<0.001$ \\
\hline
\end{tabular}

Junior residents on average took $64 \%$ longer to complete the task as compared to the experienced group $(\mathrm{p}<0.001)$. The results from each trial in Figure 4.3 reveal that both 
groups improved over time, and that the differences became smaller with additional experience on the simulator.

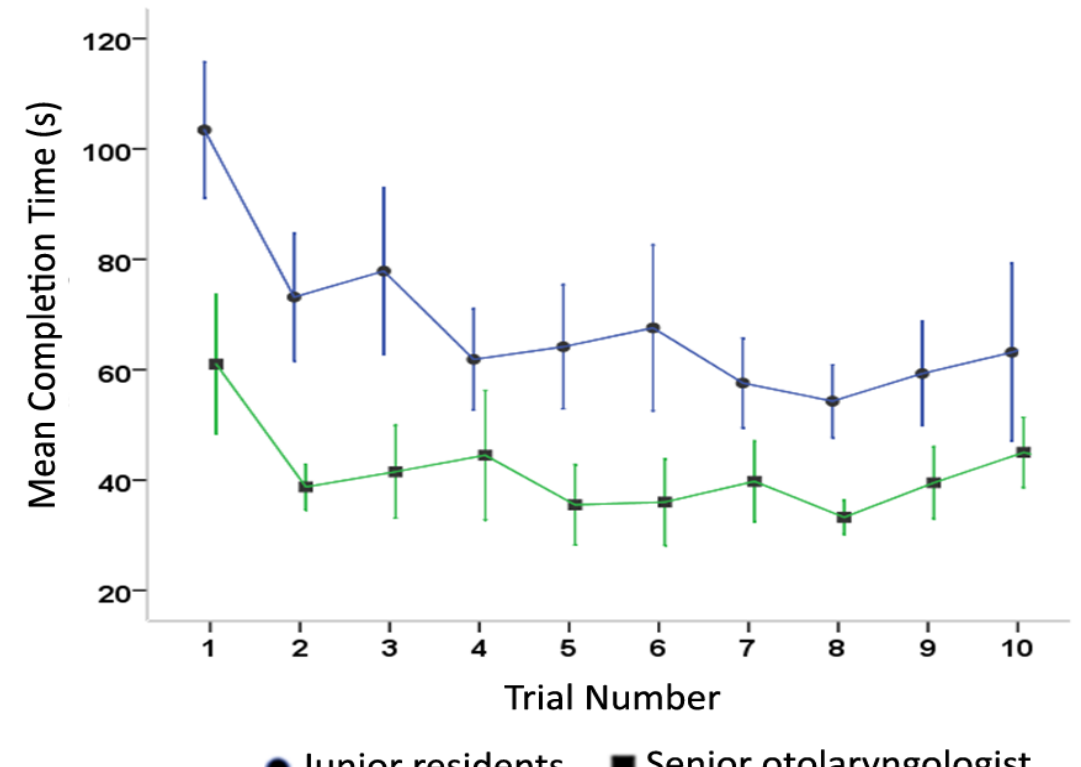

Figure 4.3 Time to completion shown for each group over 10 trials. Results are displayed as mean + /- SEM.

Senior otolaryngologists made 2.8 times fewer surgical errors as compared to the junior residents $(\mathrm{p}<0.001)$. Both groups had improvement over time as shown in Figure 4.4. When examining the types of errors made, $39 \%$ of the errors involved trauma to the external auditory canal or middle ear cavity with the myringotomy blade; $51 \%$ involved requiring multiple attempts to complete the myringotomy or tube insertion; and $10 \%$ involved placing the tube in the superior half of the tympanic membrane. 


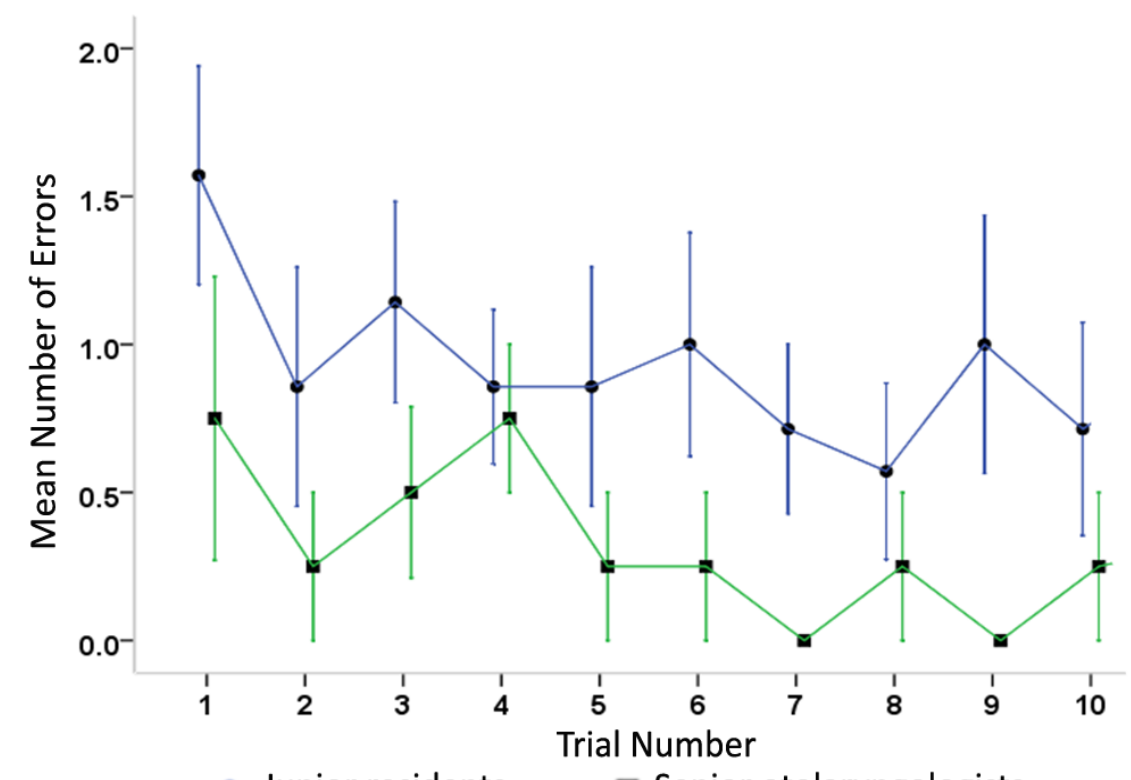

- Junior residents $\quad$ Senior otolaryngologists

Figure 4.4 Number of surgical errors shown for each group over 10 trials. Results are displayed as mean +/- SEM.

The automated analysis of the incision angle revealed that the senior otolaryngologists were able to follow the instructions and create a more accurate radial incision $(\mathrm{p}<0.002)$. However, when examining performance over time in Figure 4.5, it is clear that the primary differences occurred in the first 5 trials. After this point, the residents improved and their results were similar to the senior otolaryngologists. 


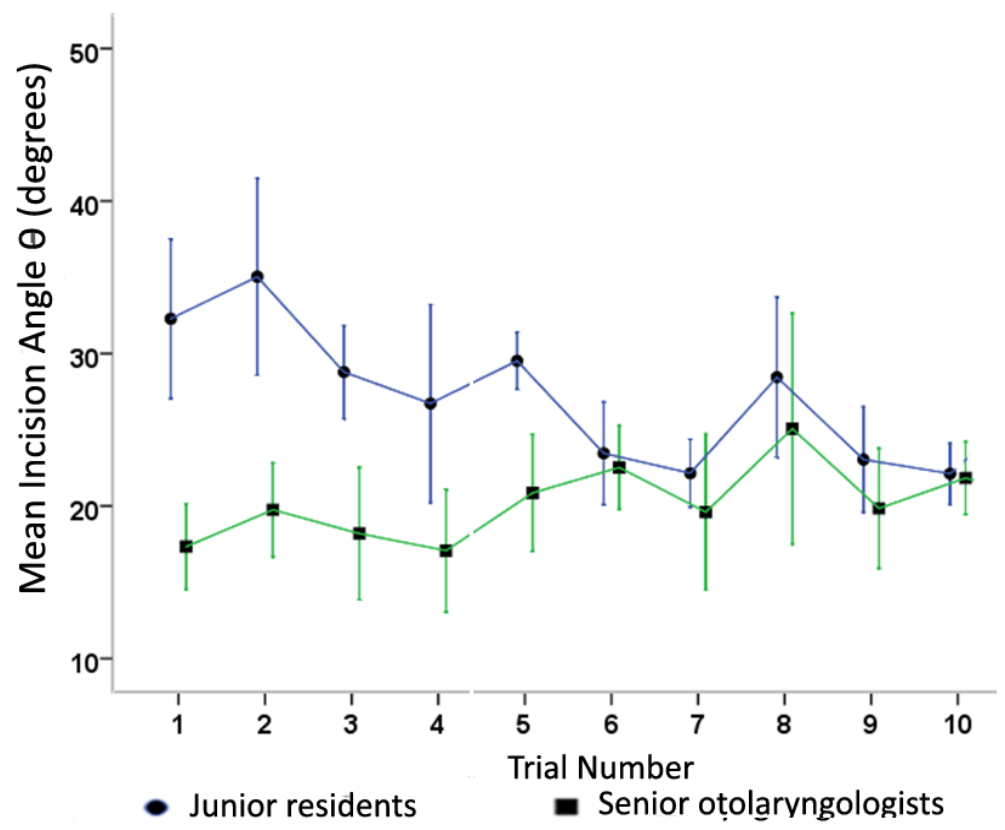

Figure 4.5 Incision angle $\theta$ shown for each group over 10 trials. Results are displayed as mean +/- SEM.

The size of the ventilation tube used in the simulator had an outer diameter of $1.5 \mathrm{~mm}$. Senior otolaryngologists tended to create $24 \%$ longer myringotomy incisions as compared to junior residents $(\mathrm{p}<0.002$ ). In reviewing each incision, $36 \%$ of the myringotomies made by the residents were smaller than $1.5 \mathrm{~mm}$, whereas this only occurred with $10 \%$ of the incisions made by senior otolaryngologists. By examining the trend over each in Figure 4.6, the experienced group tended to reduce their incision size over time, whereas the residents' incisions became slightly longer. The two groups converged in trials 9 and 10 with the myringotomy size being just larger than the tube. 


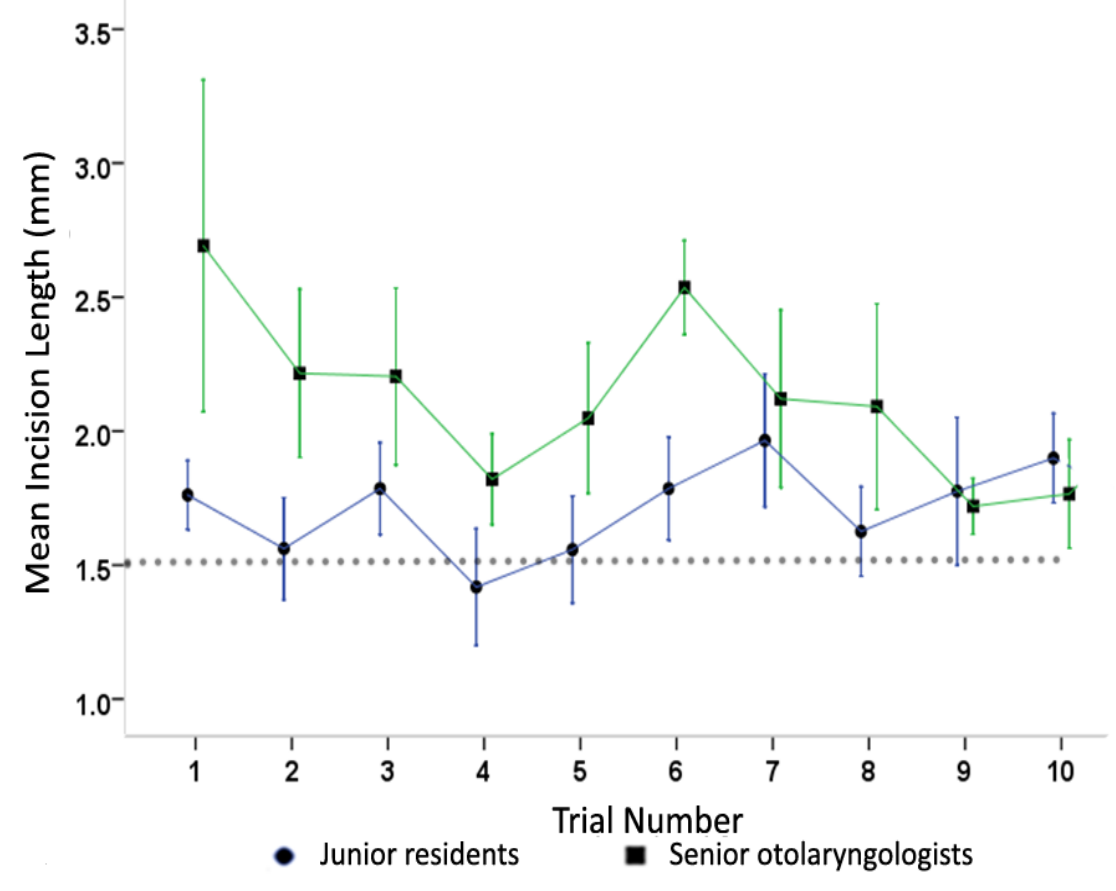

Figure 4.6 Incision length shown for each group over 10 trials. Results are displayed as mean +/- SEM.

The level of magnification of the microscope revealed one of the largest differences between the two groups $(\mathrm{p}<0.001)$. The senior otolaryngologists tended to be less zoomed in, and in all 40 myringotomies performed by the experts, both the umbo and the annulus were visible when the myringotomy was created. The residents tended to be much more magnified, and the umbo and annulus were only visible in $34 \%$ of their procedures. In Figure 4.7, both groups remained fairly stable over time with no significant improvement in the resident group. 


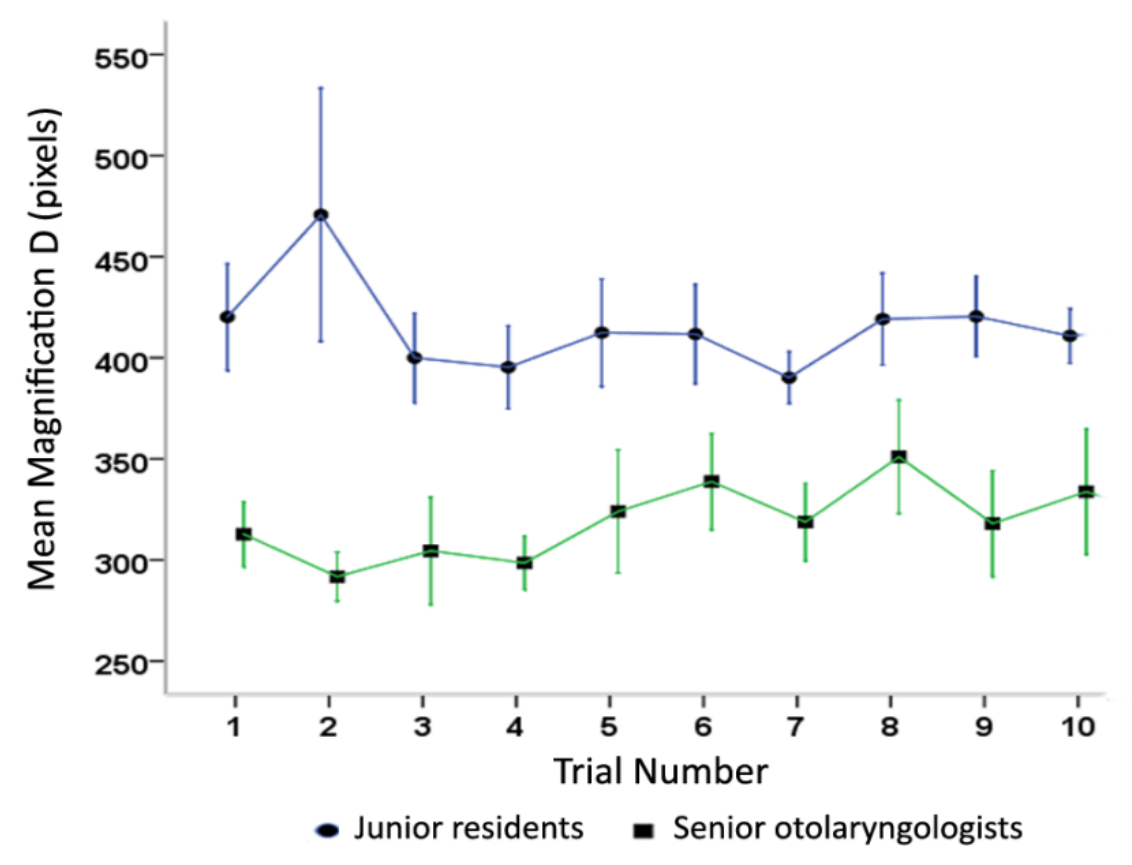

Figure 4.7 Microscope magnification level shown for each group over 10 trials. Results are displayed as mean +/- SEM.

\subsection{Discussion}

Virtual reality simulation training in Otolaryngology has now become widely accepted, and a recent systematic review by Arora et al. [34] revealed a large number of studies in temporal bone surgery and endoscopic sinus surgery. Although there are a large number of physical simulators for myringotomy and tube insertion, they are underrepresented in the realm of virtual reality and automated performance metrics [13, 34].

Volsky et al. [9] established construct validity for their physical myringotomy simulator using a non-blinded expert, however trainees required an expert to be present in order to collect metrics and provide feedback. Both Stanford University and Ohio State University have laid out comprehensive frameworks for automated simulator-based 
quantitative metrics, programming, and feedback [22, 35-36]. The data collection and computation used in the Western myringotomy simulator follows these previously published paradigms. The automated performance metrics were able to provide feedback in real-time, and accuracy was manually verified by reviewing the captured data and video playback.

Despite the small sample size, the metrics programmed were able to find significant differences between surgical residents and experts. Time to completion is an established metric that has been used to differentiate level of expertise in a number of simulators [3739]. This study also found it was a powerful metric, however it may be more appropriate to assess level of expertise rather than to provide real-time feedback as the trainee is learning the procedure.

The number of surgical errors has also been established as a solid metric to establish construct validity. Volsky et al. [9] used the following errors in their construct validity study: 1) losing the ventilation tube in the middle ear space; 2) pushing and withdrawing the tube through the myringotomy creating a "popping" sound indicating damage; 3) trauma to the external auditory canal; and 4) if the ventilation tube was dropped in the external canal. Montegue et al. [1] had 3 blinded experts rate 55 consecutive procedures and a total of 12 potential errors were identified. The four most common errors included 1) failure to perform a unidirectional incision, 2) multiple attempts to place the ventilation tube, 3) multiple attempts to complete the myringotomy, and 4) magnification of the microscope set too high. Schwartz et al. [33] conducted a blinded assessment of experts and trainees using a task based checklist. There were six items that best differentiated the participants' level of training, and of those two involved injury and 
trauma to surrounding structures during myringotomy. The surgical errors assessed Western myringotomy simulator were consistent with these studies, and this particular metric had excellent discriminant validity. It should be noted that not all of these errors necessarily led to complications for the patient (e.g. multiple attempt to insert the ventilation tube), however they provided an indication of the participants' level of expertise.

The orientation of the incision has also been used as a metric in previous studies, however differences between the groups were not significant [1,33]. The decision to instruct participants to create a radial incision in this study was based upon the authors' clinical experience, however there is debate in the literature as to whether a radial or circumferential incision is actually clinically significant [40]. In this case, the study was likely testing the participants' ability to follow instructions and technically perform the designated task. In general, experts were much more accurate in the direction of their incision, whereas residents improved and converged with the experts over time.

The accuracy of the incision orientation may be related to the microscope magnification. Having the microscope magnification setting too high was the fourth most common error found by Montague et al., [1] and microscope manipulation and positioning was found to be significantly different between groups by Schwartz et al. [33]. In this study, residents were also found to have a significantly higher microscope magnification, and the umbo and annulus were only visible in $34 \%$ of their procedures. This may have had an impact on their ability to perform a myringotomy in a radial direction and in the correct quadrant of the tympanic membrane. 
The final metric in this study was incision length, and this had previously not been shown to be significantly different between novices and experts when examining intraoperative videos [33]. In another evaluation of 55 procedures, only 2 were found to have myringotomies which were too small, and no cases where myringotomies were too large [1]. In this study, residents tended to have smaller myringotomies, and in $36 \%$ of the cases the myringotomy was smaller than the diameter of the tube. Conversely, experts started with much larger myringotomies, and the size decreased over time until it was just larger than the ventilation tube in the final trials. As the two groups converged, the discrimination validity of this metric would be most useful during the initial stages of simulator use.

Although the Western myringotomy simulator now has automated performance metrics and established construct validity, this does not ensure that residents will obtain better intraoperative performance after using the simulator [41-42]. A virtual reality to operating room skills transference study will be needed to fully assess the utility of the simulator in a clinical setting. Future work on the simulator includes defining metrics around operating microscope usage and implementing them into the simulator. The authors plan to expand the soft-tissue deformation module to include other otologic procedures such as tympanomeatal flaps, tympanoplasty, and ossiculoplasty. Finally, and multi-centred study is planned to formally assess whether the skills gained by using the simulator can be applied to the operating room setting. 


\subsection{Conclusion}

The Western myringotomy simulator has previously demonstrated face and content validity. Automated quantitative performance metrics were successfully developed and implemented into the simulator. The performance metrics successfully discriminated between expert and novice subjects, thereby establishing construct validity. Further studies will be needed to establish skills transference from the simulator to the operating room. 


\subsection{References}

1 M. L. Montague, M. S. W. Lee, S. S. M. Hussain, "Human error identification: An analysis of myringotomy and ventilation tube insertion," Archives of Otolaryngology-Head and Neck Surgery, vol. 130, no. 10, pp. 1153-1157, 2004.

2 B. N. Brodish, A. L. Woolley, "Major vascular injuries in children undergoing myringotomy for tube placement," American Journal of Otolaryngology, vol. 20, pp. 46-50, 1999.

3 M. Kumar, A.M. Khan, S. Davis, "Medial displacement of grommets: an unwanted sequel of grommet insertion," The Journal of Laryngology \& Otology, vol. 114, pp. 448-449, 2000.

4 J. C. Groblewski, E. H. Harley, "Medial migration of tympanostomy tubes: an overlooked complication," International Journal of Pediatric Otorhinolaryngology, vol. 70, pp. 1707-1714, 2006.

5 T. Walker, S. Duvvi, and B. N. Kumar, "The Wigan Grommet Trainer," Clinical Otolaryngology, vol. 31, no. 4, pp. 349-350, 2006.

6 M. Duijvestein, and J. Borgstein, "The Bradford Grommet Trainer," Clinical Otolaryngology, vol. 31, no. 2, pp. 163-163, 2006.

7 A. Leong, S. Kundu, P. Martinez-Devesa, C. Aldren, "Artificial ear: a training tool for grommet insertion and manual dexterity," ORL : Journal for Oto - Rhino Laryngology and Its Related Specialties, vol. 68, no. 2, pp. 115-117, 2006.

8 P. Hong, A. N. Webb, G. Corsten, J. Balderston, R. Haworth, K. Ritchie, E. Massoud. "An anatomically sound surgical simulation model for myringotomy and tympanostomy tube insertion," International Journal of Pediatric Otorhinolaryngology, vol 78, pp. 522-529, 2008.

9 P. G. Volsky, B. B. Hughley, S. M. Peirce, B. W. Kesser, "Construct validity of a simulator for myringotomy with ventilation tube insertion," Otolaryngology-Head and Neck Surgery, vol. 141, pp. 603-608, 2009.

10 P. K. Singh, M. De, R. Vaughan-Jones, "A model for training in grommet insertion," Annals of The Royal College of Surgeons of England, vol. 87, no. 4, pp. 287-288, 2005.

11 E. R. Carr, E. Benjamin, "'Surgical skills box': a new training aid for surgical trainees, " The Journal of Laryngology \& Otology, vol. 120, no. 2, pp. 133-134, 2006.

12 S. Malekzadeh, G.Hann, B. Wilson, M. Pehlivanova, G. Milmoe, "A model for training and evaluation of myringotomy and tube placement skills," The Laryngoscope, vol. 121, no. 7, pp. 1410-1415, 2011.

13 S. Mahalingam, Z. Awad, N. S. Tolley, S. Khemani, "Ventilation tube insertion simulation: A literature review and validity assessment of five training models," Clinical Otolaryngology, doi: 10.1111/coa.12543, 2015 Sep 19.

14 M. Nagendran, K. S. Gurusamy, R. Aggarwal, M. Loizidou, B. R. Davidson, "Virtual reality training for surgical trainees in laparoscopic surgery," Cochrane Database of Systematic Reviews, doi:10.1002/14651858.CD006575.pub3, 2013 Aug 27. 
15 S. Weghorst, C. Airola, P. Oppenheimer, C. V. Edmond, T. Patience, D. Heskamp, J. Miller, "Validation of the Madigan ESS Simulator," Studies in Health Technology and Informatics, vol. 50, pp. 399-405, 1998.

16 S. M. Anil, Y. Kato, M. Hayakawa, K. Yoshida, S. Nagahisha, T. Kanno, "Virtual 3dimensional preoperative planning with the Dextroscope for excision of a 4th ventricular ependymoma," Minimally Invasive Neurosurgery, vol. 50, pp. 65-70, 2007.

17 M. Audette, H. Delingette, A. Fuchs, O. Astley, K. Chinzei, "A topologically faithful, tissue-guided, spatially varying meshing strategy for computing patient-specific head models for endoscopic pituitary surgery simulation," Studies in Health Technology and Informatics, vol. 119, pp. 22-27, 2006.

18 B. Tolsdorff, A. Pommert, K. H. Höhne, A. Petersik, B. Pflesser, U. Tiede, R. Leuwer, "Virtual reality: a new paranasal sinus surgery simulator," Laryngoscope, vol. 120, pp. 420-426, 2010.

19 R. Varshney, S. Frenkiel, L. H. Nguyen, M. Young, D. R. Maestro, A. Zeitouni, M. A. Tewfik, "Development of the McGill simulator for endoscopic sinus surgery: a new high-fidelity virtual reality simulator for endoscopic sinus surgery," The American Journal of Rhinology \& Allergy, vol. 28, no. 4, pp. 330-334, 2014.

20 G. J. Wiet, D. Stredney, T. Kerwin, B. Hittle, S. A. Fernandez, M. Abdel-Rasoul, D. B. Welling, "Virtual temporal bone dissection system: development and testing," The Laryngoscope, vol. 122(Suppl. 1), pp. S1-S12, 2012.

21 D. Morris, C. Sewell, F. Barbagli, K. Salisbury, N. H. Blevins, S. Girod, "Visuohaptic simulation of bone surgery for training and evaluation," IEEE Computer Graphics and Applications, vol. 26, pp. 48-57, 2006.

22 C. Sewell, D. Morris, N. H. Blevins, S. Dutta, S. Agrawal, F. Barbagli, K. Salisbury, "Providing metrics and performance feedback in a surgical simulator," Computer Aided Surgery, vol. 13, pp. 63-81, 2008.

23 A. Arora, S. Khemani, N. Tolley, A. Singh, J. Budge, D. A. Varela, H. W. Francis, A. Darzi, N. I. Bhatti, "Face and content validation of a virtual reality temporal bone simulator," Otolaryngology-Head and Neck Surgery, vol. 146, pp. 497-503, 2012.

24 P. Piromchai , A. Avery, M. Laopaiboon, G. Kennedy, S. O'Leary, "Virtual reality training for improving the skills needed for performing surgery of the ear, nose or throat," Cochrane Database of Systematic Reviews, doi: 10.1002/14651858.CD010198.pub2, 2015 Sep 9.

25 S. Khemani, A. Arora, A. Singh, N. Tolley, A. Darzi, "Objective skills assessment and construct validation of a virtual reality temporal bone simulator," Otology \& Neurotology, vol. 33, no. 7, pp. 1225-1231, 2012.

26 Y. C. Zhao, G. Kennedy, R. Hall, S. O'Leary, "Differentiating levels of surgical experience on a virtual reality temporal bone simulator," Otolaryngology-Head and Neck Surgery, vol. 143, no. 5 (Supplement), pp. S30-S35, 2010.

27 M. P Fried, B. Sadoughi, S. J. Weghorst, M. Zeltsan, H. Cuellar, J. I. Uribe, C. T. Sasaki, D. A. Ross, J. B. Jacobs, R. A. Lebowitz, R. M. Satava, "Construct validity of the endoscopic sinus surgery simulator simulator II. Assessment of discriminant validity and expert benchmarking," Archives of Otolaryngology - Head \& Neck Surgery, vol. 133, no. 4, pp. 350-357, 2007. 
28 R. Varshney, S. Frenkiel, L. H. Nguyen, M. Young, D. R. Maestro, A. Zeitouni, M. A. Tewfik, "Development of the McGill simulator for endoscopic sinus surgery: a new high-fidelity virtual reality simulator for endoscopic sinus surgery," The American Journal of Rhinology \& Allergy, vol. 28, no. 4, pp. 330-334, 2014.

29 B. Wheeler, P. C. Doyle, S. Chandarana, S. Agrawal, M. Husein, H. M. Ladak, "Interactive computer-based simulator for training in blade navigation and targeting in myringotomy," Computer Methods Programs in Biomedicine, vol. 98, no. 2, pp. 130-139, 2010.

30 A. K. Ho, H. Alsaffar, P. C. Doyle, H. M. Ladak, S. K. Agrawal, "Virtual reality myringotomy simulation with real-time deformation: development and validity testing," The Laryngoscope, vol. 122, no. 8, pp. 1844-1851, 2012.

31 C. Huang, S. K. Agrawal, H. M. Ladak, "Virtual-reality simulator for training in myringotomy with tube placement," Proceedings of the 37th Canadian Medical and Biological Engineering Conference, May 2014.

32 C. Huang, H. Cheng, Y. Bureau, S. K. Agrawal, H. M. Ladak, "Face and content validity of a virtual-reality simulator for myringotomy with tube placement ," Journal of Otolaryngology - Head \& Neck Surgery, doi: 10.1186/s40463-015-0094-2, 2015 Oct 20.

33 J. Schwartz, A. Costescu, M. A. Mascarella, M. E. Young, M. Husein, S. Agrawal, K. Roth, P. C. Doyle, L. H. Nguyen, "Objective assessment of Myringotomy and tympanostomy tube insertion: A prospective single-blinded validation study, "The Laryngoscope, doi: 10.1002/lary.25746, 2015 Oct 26.

34 A. Arora, L. Y. Lau, Z. Awad, A. Darzi, A. Singh, N. Tolley, "Virtual reality simulation training in Otolaryngology, "International Journal of Surgery, vol. 12, no. 2, pp. 87-94, 2014.

35 T. Kerwin, G. Wiet, D. Stredney, H. W. Shen, "Automatic scoring of virtual mastoidectomies using expert examples," The International Journal for Computer Assisted Radiology and Surgery, vol. 7, no. 1, pp. 1-11, 2012.

36 G. Wiet, B. Hittle, T. Kerwin, D. Stredney, "Translating surgical metrics into automated assessments," Studies in Health Technology and Informatics, vol. 173, pp. 543-548, 2012.

37 C. Lyons, D. Goldfarb, S. L. Jones, N. Badhiwala, B. Miles, R. Link, B. J. Dunkin, "Which skills really matter? proving face, content, and construct validity for a commercial robotic simulator, " Surgical Endoscopy, vol. 27, no. 6, pp. 2020-2030, 2013.

38 P. Piromchai, A. Avery, M. Laopaiboon ,G. Kennedy, S. O'Leary, "Virtual reality training for improving the skills needed for performing surgery of the ear, nose or throat," Cochrane Database of Systematic Reviews, doi: 10.1002/14651858.CD010198.pub2, 2015 Sep 9.

39 M. Nagendran, K. S. Gurusamy, R. Aggarwal, M. Loizidou , B. R. Davidson, "Virtual reality training for surgical trainees in laparoscopic surgery," Cochrane Database of Systematic Reviews, doi: 10.1002/14651858.CD006575.pub3, 2013 Aug 27.

40 M. D. Guttenplan, L. W. Tom, M. A. DeVito, S. D. Handler, R. F. Wetmore, W. P. Potsic, "Radial versus circumferential incision in myringotomy and tube placement," 
International Journal of Pediatric Otorhinolaryngology, vol. 21, no. 3, pp. 211-215, 1991.

41 A. G. Gallagher, E. M. Ritter, R. M. Satava, "Fundamental principles of validation, and reliability rigorous science for the assessment of surgical education and training," Surgical Endoscopy, vol. 17, no. 10, pp. 1525-1529, 2003.

42 B. M. A. Schout, A. J. M. Hendrikx, F. Scheele, B. L. H. Bemelmans, A. J. J. A. Scherpbier, "Validation and implementation of surgical simulators: a critical review of present, past, and future," Surgical endoscopy, vol. 24, no. 3, pp. 536-546, 2010. 


\section{Chapter 5. \\ Summary and Future Directions}

\subsection{Summary}

\subsubsection{Simulator design and implementation}

As described in chapter 2, a complete VR myringotomy simulator has been developed. The simulated scenarios include 1) the surgical instruments such as myringotomy blade, forceps, ventilation tube, ear speculum and microscope, 2) the human ear including the auricle, ear canal and eardrum, 3) microscope binocular (stereoscopic) vision, 4) positioning and operating of the surgical instruments in 3D space, 5) eardrum deformation, 6) cutting of the eardrum, 7) ventilation tube insertion, and 7) tactile feedback when the surgical instruments touch the ear canal, cut the eardrum and when the tube is inserted.

The simulated anatomy was derived from clinical high resolution CT images. The Unity 3D digital game engine was used as the software development platform for real-time graphical rendering, subdivision cutting and physics simulation. A haptic arm co-located with the stereo scene was used to manipulate virtual surgical tools and to interact with the virtual ear with force feedback. A unique collision model was successfully implemented in the simulation, in which ball-shaped collision detectors served for detecting contacts between the virtual surgical tools and the virtual eardrum and then deforming the eardrum, while the mesh collision detector was used for detecting contacts between the blade and the dynamic eardrum mesh and then activating the cutting algorithm. The 
cutting algorithm was based on the subdivision method and permits cutting to be performed at realistic speeds. System performance was evaluated by changing the physics calculation rate while observing the variation of the display frame rate. When the physics calculation rate was set between 23.5 and $37 \mathrm{~Hz}$, the corresponding display frame rate (FR) varied from 23.5 to $20 \mathrm{~Hz}$. It was verified that this enables a stable physics calculation so as to achieve both force feedback and smooth display, and met the realtime interactive requirement (frame rates 20 to 30 frames per second [1]) for the surgical manipulation.

\subsubsection{Face and content validity study}

A face and content validity study were conducted using a questionnaire. The questionnaire was developed in consultation with instructing surgeons. Fourteen face validity questions were developed that focused on the anatomy of the ear, simulation of the surgical microscope, appearance and movement of the surgical instruments, deformation and cutting of the eardrum, and tube insertion. Six content validity questions were developed that focused on training potential on surgical tasks such as speculum placement, microscope positioning, tool navigation, ear anatomy, myringotomy creation and tube insertion. A total of 12 participants from the Department of Otolaryngology Head and Neck Surgery (Western University) were recruited for the study.

Responses to 12 of the 14 questions on face validity were predominantly positive. With regard to content validity, $64 \%$ of the responses were positive, $21 \%$ were neutral, and $15 \%$ were negative. One issue of concern was with collision detection modeling related to tube insertion into the eardrum, and the second was with the movement of the blade and 
forceps, both of which can easily be remedied. The myringotomy simulator appears to have sufficient face and content validity.

\subsubsection{Construct validity study}

The construct validity study involved two groups of users consisting of four senior Otolaryngologists and seven junior Otolaryngology residents. Their skills were evaluated using five automated metrics, including time to completion, surgical errors, incision length, incision angle and the magnification of the microscope. The analysis of all ten trials combined showed their skills were differentiated by the simulator by all five metrics. As expected, the experts demonstrated superior performance with respect to all metrics; however, there tended to be an improvement in the performance of residents with continued use of the simulator.

\subsection{Discussion and future directions}

\subsubsection{Simulator design and implementation}

A simulation system combines a number of sub-systems including those for graphical rendering, tissue deformation modeling and tactile feedback. In the simulator design, calculation models and algorithms are selected and implemented so that the calculation load is well balanced to obtain real-time performance while retaining realism.

The selection of the mass-spring method to model eardrum deformation and the subdivision cutting algorithm for incision simulation was in part because their calculation load is relatively low. The collision detection model separated the calculation of the cutting and eardrum deformation through the use of two collision detectors. The Unity3D 
digital engine was selected as it is a well-organized development platform with excellent rendering, collaboration of objects, and support for a physics engine - PhysX.

In Chapter 2, an optimization experiment has been performed to balance the rendering speed, the calculation of eardrum deformation and the haptics. In terms of the calculation loading, the most time consuming step is the calculation of eardrum deformation. The calculation time was highly correlated with the number of node points (collision detectors) distributed on the mass-spring network. Testing on a personal computer indicated that increasing the density of the nodes could dramatically decrease the performance of the system in terms of speed and impeding the performance of other algorithms such as cutting calculation and visual and haptic rendering. By contrast, reducing the number of the nodes could improve the rendering speed and shorten the time for deformation calculation. However, this inevitably can decrease the accuracy of the collision detection. In the current simulation, seven hundred and eighty nodes (small ball-shaped collision detectors) were distributed on the eardrum surface; there were still small gaps between the ball-shaped collision detectors. These gaps can disable the deformation calculation when an instrument touches the eardrum in the gaps. This caused unrealistic representation of splaying during the tube insertion into the incision, which was evident in response to questions 9 and 13 of the face and content validity study.

The situation could be improved by using a higher resolution collision model, e.g., increasing the density of the nodes in the models. This would improve the accuracy of contact detection between the virtual eardrum and surgical instruments, but this would require more processing power, which is attainable using current computers. 
In regard to the hardware setup, users indicated that the motion of the virtual blade and forceps was occasionally not stable. In the simulation, the movements of the surgical tools were controlled by the handle of the Omni haptic arm. The haptic arm is an electromechanical device with mechanical linkages to the handle. When moving the surgical instrument by the handle, the user can feel some stiffness due to the mechanical friction from the joints of the linkage. Moreover, the shape of the handle was like a cylinder whose diameter is much bigger than the shaft of real surgical tools. Both issues result in the handle not feeling like a real-life instrument and lead to unstable movement of the virtual instruments.

One possible solution to increase the stability of the instruments discussed in Chapter 2 was change to a higher fidelity device with substantially reduced friction (e.g., Geomagic Phantom Premium device from Geomagic, Inc., Morrisville, NC) and modifying the handle of the device to match a standard surgical instrument as demonstrated in related literature [2]. A further consideration could be providing force feedback from the surface of the speculum so that additional stability can be achieved when the virtual blade moves forward against the surface of the virtual speculum. However, given that in the current simulator the calculation load has plateaued, enabling force feedback for the speculum is not suggested as it will substantially slow down graphical and haptic rendering.

A hybrid approach introducing a real speculum and a real blade connected to the handle of the haptic arm is an alternative way to model interactions between the blade and speculum while avoiding extra calculation load. Components such as the eardrum and ear canal would still be represented digitally as flexibility is required when modeling anatomical variability. This hybrid method combining some physical and some virtual 
components requires the designer to co-locate the virtual scene (virtual blade and virtual speculum) with the real scene (real speculum and real blade) by highly accurate registration. In a feasibility experiment, the Omni haptic arm was modified and calibrated using the method reported in [3], which was applied to calibrate Phantom Premium haptic devices. The test results showed that the positioning accuracy of the employed Omni haptic arm was not enough for the registration accuracy required.

Another hybrid solution could be a combination of a physical replica head and a highprecision tracking device. Haptics would be provided because of the interaction between physical components. For example, the NDI (Northern Digital Inc., Waterloo, ON) electromagnetic tracker can act as a tracking device instead of haptic arm for the tracking of instruments. The tracked tool can then be moved freehand without a mechanical connection to the tracking device. A corresponding virtual tool would be rendered in the VR scene to mimic the movements of the actual tool. A real speculum could be set in the ear of a physical replica head. The actual speculum and device handle can be registered to the virtual speculum and blade and co-located when the simulation is started. This setting would better mimic the actual surgical approach. For instance, it would permit the trainee to rest the fingers of the operating hand on the replica head to help reduce hand tremor. It would also allow easier guidance of the blade towards the eardrum at an appropriate angle as this would be guided by a physical speculum. A shortcoming of this solution is that the tracking device is not able to provide tactile feedback when contact is made with virtual objects such as the eardrum and ear canal models. 


\subsubsection{Face validity study}

In the face validity study, a 7-point Likert scale was used to answer each question. As further analysis, the intra-observer consistencies across the participants within each group were analyzed using intraclass correlation. The intraclass correlation coefficient (ICC) computed for the group of residents was 0.11 , indicating that $11 \%$ of the variation is between the questions, whereas $89 \%$ is between residents. The ICC for senior Otolaryngologists was 0.22 . The ICC suggests that there is little agreement between residents or between experts when responding to the questions in the survey. A satisfactory ICC would normally be approximately 0.80 or above. It should be noted though that this poor result may be due to the restricted range of the scale, which is used

to categorize the responses. A fine scale may go beyond the raters' resolving power [4, 5]. It is possible that if the scale was expanded there would be a greater agreement between the users. Nevertheless, this result is interesting and may be more indicative of questionnaire design than of simulator problems, e.g., using 5-point Likert scale instead of current 7-point Likert scale to answer each question.

\subsubsection{Metrics and construct validity}

In the construct validity study (Chapter 4), the binary definition of metrics (e.g., measurement of error) poses a rigid classification problem. A further improvement could be to let the software record more details of the operation and replace the binary decision by a continuous scale. Such continuous metrics may be better able to represent trainees at various stages of learning. Furthermore, artificial intelligence classification techniques such as Hidden Markov Models and Naïve Bayes Classifier could be used to determine trainee stage [6]. Furthermore, validated metrics need to be formulated into a scoring 
system to evaluate users' performance as a whole. For example, based on validated metrics, a rule-based fuzzy inference model can be applied to design a scoring system so that user can get a final performance score after each training session [7]. 


\subsection{Reference}

1 G. J. Wiet, D. Stredney, T. Kerwin, B. Hittle, S. A. Fernandez, M. Abdel-Rasoul, D. B. Welling, "Virtual temporal bone dissection system: development and testing," Laryngoscope, vol. 122(Suppl. 1), pp. S1-S12, 2012.

2 T. R. Coles, N. W. John, G. Sofia, D. A. Gould, D. G. Caldwell, "Modification of commercial force feedback hardware for needle insertion simulation," MMVR18 Medicine Meets Virtual Reality 2011 Poster: 8-12 February 2011, Newport Beach, CA.

3 M. Harders, G. Bianchi, B. Knoerlein, G. Szekely, "Calibration, registration, and synchronization for high precision augmented reality haptics," IEEE Transactions on Visualization and Computer Graphics, vol. 15, no. 1, pp. 138-149, 2009.

4 W. R. Garner, R. Wendell, W. H. Harold, "The Amount of Information in Absolute Judgments," Psychological Review, vol. 58, no. 6, pp. 446-459, 1951.

5 J. Jacoby and S. M. Matell, "Three-Point Likert Scales Are Good Enough," Journal of Marketing Research, vol. 8, no. 4, pp. 495-500, 1971.

6 C. Sewell, D. Morris, N. H. Blevins, S. Dutta, S. Agrawal, F. Barbagli, K. Salisbury, "Providing metrics and performance feedback in a surgical simulator," Computer Aided Surgery, vol. 13, no, 2, pp. 63-81, 2008.

7 I. Hajshirmohammadi, S Payandeh, "Fuzzy set theory for performance evaluation in a surgical simulator," Presence: Teleoperators \& Virtual Environments, vol. 16, no. 6, pp. 603-622, 2007. 


\section{Appendix A: Ethics Approval Notice}

\section{Research Western University Health Science Research Ethics Board HSREB Delegated Initial Approval Notice}

Principal Investigator: Prof. Hanif Ladak

Department \& Institution: Schulich School of Medicine and Dentistry, Western University

HSREB File Number: 105239

Study Title: Face and content validity of a computer-based simulator for training in myringotomy with tube insertion

Sponsor:

HSREB Initial Approval Date: June 03, 2014

HSREB Expiry Date: May 31, 2015

Documents Approved and/or Received for Information:

\begin{tabular}{|l|l|l|}
\hline Document Name & Comments & Version Date \\
\hline Instruments & Questionnaire & $2014 / 04 / 14$ \\
\hline Western University Protocol & Revised protocol - clean version & $2014 / 05 / 13$ \\
\hline Letter of Information \& Consent & Merged LOI and consent form. Clean copy. & $2014 / 06 / 03$ \\
\hline
\end{tabular}

The Western University Health Science Research Ethics Board (HSREB) has reviewed and approved the above named study, as of the HSREB Initial Approval Datenoted above.

HSREB approval for this study remains valid until the HSREB Expiry Date noted above, conditional to timely submission and acceptance of HSREB ContinuingEthics Review. If an Updated Approval Notice is required prior to the HSREB Expiry Date, the Principal Investigator is responsible for completing andsubmitting an HSREB Updated Approval Form in a timely fashion.

The Western University HSREB operates in compliance with the Tri-Council Policy Statement Ethical Conduct for Research Involving Humans (TCPS2), theInternational Conference on Harmonization of Technical Requirements for Registration of Pharmaceuticals for Human Use Guideline for Good Clinical PracticePractices (ICH E6 RI), the Ontario Personal Health Information Protection Act (PHIPA, 2004), Part 4 of the Natural Health Product Regulations, Health CanadaMedical Device Regulations and Part C, Division 5, of the Food and Drug Regulations of Health Canada.

Members of the HSREB who are named as Investigators in research studies do not participate in discussions related to, nor vote on such studies when theyare presented to the REB.

The HSREB is registered with the U.S. Department of Health \& Human Services under the IRB registration number IRB 00000940 . 


\section{Curriculum Vitae}

\section{PROFESSIONAL EXPERIENCE}

Software Engineer

Phoenix Interactive Design (Diebold).
04/2015 -Now

London, Canada

- Developing ATM software (C++, MKS)

- Maintaining existing software VISTA by making modifications

\section{Software Developer}

$04 / 2010-08 / 2010$

Magnetic Resonance Innovations Canada Inc. Windsor, Canada

- Developed three software components for Magnetic Resonance Image Processing using C++, MFC, MS Visual Studio 2008, SVN

- Designed and wrote command line programs for existing VC MFC procedures

- Maintained existing software SPIN

\section{Research Associate}

01/2008-07/2009

Department of Electrical and Computer Engineering

University of Alberta.

Edmonton, Canada

- Developed a method with a new mathematical model to analyze stock market price fluctuations using Fuzzy Clustering, Real-code Genetic Algorithm

- Designed and developed software to implement the model using C\#.NET, Excel, SQL- Server

Software Developer and Assistant Professor

$05 / 2005-12 / 2007$

High Tech Corporation of KMUST, Department of Computer Science

Kunming University of Science and Technology (KMUST). Kunming, China

- Developed a "Cast Analysis System" for Yunnan Post Office using ASP.NET, c\#, B/S structure

- Developed a "Television Assessment System" for Yunnan TV network using ASP.NET, c\#, B/S structure

- Implemented "Managerial Report System" for Yunnan TV using VBA, Excel

- Participated as a team member in "Iris-Based Identity Authentication System" in the Lab of Pattern Recognition using VC++

- Taught C Programming, Fundamentals of Computers, Database Applications and Computer Networking 
Kunming Yueye Science and Technology Company Ltd.

Kunming, China

- Wrote, modified, integrated and tested software codes in C

- Maintained existing computer systems

- Participated as a team member in a workflow software development for Kunming Cigarette Factory; wrote a 2D virtual animation to describe process flow using $\mathrm{C}$

Civil Engineer /Manager

05/1992-12/1997

Construction Department

Yunnan Tong Cheng Exploiting Company.

Kunming, China

- Designed civil engineering plans

- Managed construction projects including all construction progress and

- Subsystems

- Implemented 3-D virtual models for architectural designs

\section{EDUCATION}

PhD Student majoring in Software Engineering

09/2011 -Now

The University of Western Ontario, Electrical \& Computer Engineering London, Canada

Masters of Engineering in Computer Science

$05 / 2005$

Kunming University of Science and Technology Kunming, China

Associate Degree in Computer Science

05/1995

Hefei University of Technology

Hefei, China

Degree in Civil Engineering

$07 / 1991$

HuaZhong University of Science and Technology Wuhan, China

\section{SCHOLARSHIPS}

2014: Mitacs Accelerate graduate internship ( $\$ 15,000$ for 6 -month internship)

2011-2013: Natural Sciences and Engineering Council CREATE program in Computer Assisted Medical Interventions graduate scholarship $(\$ 15,000 / \mathrm{yr})$.

2011-2015: Western Graduate Research Scholarship, Western University, Electrical and Computer Engineering Graduate Program (variable, $\approx \$ 6,750 / \mathrm{yr}$ ). 


\section{PUBLICATIONS}

\section{Peer-Reviewed Journal Papers (Published, accepted, or under review)}

1. C. Huang, S. K. Agrawal, H. M. Ladak, "A virtual-reality simulator for training in myringotomy with tube placement," Journal of Medical and Biological Engineering, accepted April, 2015.

2. C. Huang, H. Cheng, Y. Bureau, S. K. Agrawal, H. M. Ladak, "Face and content validity of a virtual-reality simulator for myringotomy with tube placement," Journal of OtolaryngologHead and Neck Surgery, accepted October, 2015.

3. C. Huang, H. Cheng, Y. Bureau, H. M. Ladak, S. K. Agrawal, "Automated quantitative metrics in a virtual-reality myringotomy and tube simulator: Development and construct validity, The Laryngoscope, Submitted in December, 2015.

\section{Peer-Reviewed Conference Proceedings}

1. H. M. Ladak, C. Huang, H. Cheng, S. K. Agrawal, "Face validity of a virtual-reality simulator for training in myringotomy with tube placement," Middle-Ear Mechanics in Research and Otology, $7^{\text {th }}$ International Symposium, Aalborg, Denmark, July 2015, pp. 71-72.

2. C. Huang, S. K. Agrawal, H. M. Ladak, "Virtual-reality simulator for training in myringotomy with tube placement," Proc. $37^{\text {th }}$ Canadian Medical \& Biological Engineering Conference, Vancouver, BC, May 2014. 